\title{
Dois problemas de busca
}

\author{
Renato Carmo
}

\author{
TESE APRESENTADA \\ $A O$ \\ INSTITUTO DE MATEMÁTICA E ESTATÍSTICA \\ DA \\ UNIVERSIDADE DE SÃO PAULO \\ PARA \\ OBTENÇÃO DO GRAU DE DOUTOR \\ EM \\ CIÊNCIAS
}

Área de Concentração: Ciência da Computação

Orientador: Prof. Dr. Yoshiharu Kohayakawa

Durante a elaboração deste trabalho o autor recebeu afastamento do Departamento de Informática da Universidade Federal do Paraná e apoio financeiro do Programa PICDT da CAPES.

São Paulo, junho de 2005 


\title{
Dois problemas de busca
}

\author{
Este exemplar corresponde à redação final da \\ tese devidamente corrigida e defendida \\ por Renato Carmo \\ e aprovada pela Comissão Julgadora.
}

São Paulo, 22 de junho de 2005.

\section{Banca examinadora:}

Prof. Dr. Yoshiharu Kohayakawa (orientador) (IME-USP)

Prof. Dr. Eduardo Sany Laber (DINF-PUC-Rio)

Prof. Dr. Jayme Luiz Szwarcfiter (COPPE-UFRJ)

Prof. Dr. Flávio Keidi Miyazawa (IC-UNICAMP)

Prof. Dr. Jozef Skokan (IME-USP) 


\title{
Resumo
}

Dois problemas de busca são estudados: o problema de busca num conjunto parcialmente ordenado e o problema de otimização de consultas em bases de dados com predicados caros. Provamos que o primeiro problema é $\mathcal{N} P$-difícil e apresentamos algoritmos polinomiais que fornecem aproximações de maneira assintoticamente quase certa. Para o segundo problema cotas justas inferiores de desempenho são apresentadas para algoritmos determinísticos e aleatorizados, bem como algoritmos determinísticos e aleatorizados que atingem ou aproximam essas cotas. Diversas variantes do problema são consideradas.

\begin{abstract}
We study two search problems: the problem of searching on a partially ordered set and the problem of optimizing database queries with expensive predicates. We show that the first problem is $\mathcal{N} P$-hard and we introduce polynomial time algorithms which give approximate solutions assymtoptically almost surely. For the second problem, tight lower bounds on the performance of deterministic and randomized algorithms are proved. We also introduce deterministic and randomized algorithms which meet or approximate the given bounds. Several variations of the problem are considered.
\end{abstract}


A meus pais, Paulo e Terezinha, que me ensinaram as coisas realmente importantes. 


\section{Sumário}

$\begin{array}{ll}\text { Introdução } & 1\end{array}$

Preliminares $\quad 3$

1 Notação, Convenções e Algumas Definições . . . . . . . . . . 3

2 Problemas de Busca Ótima e Jogos de Esconder . . . . . . 4

I Busca Ótima em Ordens Parciais 11

$\begin{array}{ll}\text { Preliminares } & 13\end{array}$

Grafos .......................... 13

Ordens . . . . . . . . . . . . . . . . . 13

Modelos Probabilísticos . . . . . . . . . . . . . 15

$\begin{array}{ll}\text { Introdução } & 17\end{array}$

Organização do Texto . . . . . . . . . . . . . . . 18

Créditos ..................... 20

1 Complexidade Computacional 21

2 Ordens Aleatórias: Modelos Probabilísticos para Ordens 29

2.1 O Modelo Grafo-Aleatório . . . . . . . . . . . . . . . . . . 30

2.2 O Modelo Uniforme . . . . . . . . . . . . . . . . . . . 33

3 Busca Ótima em Ordens Grafo-Aleatórias 37 
4 Busca Ótima em Ordens Uniformes 43

4.1 Busca em Ordens Tripartidas . . . . . . . . . . . . . . . 44

5 Um Problema Correlato $\quad 47$

6 Comentários Finais $\quad 49$

II Consulta de Informação Cara em Bases de Dados 51

Preliminares $\quad 53$

A Técnica Minimax de Yao . . . . . . . . . . . . . 54

Introdução $\quad 57$

1 Formalização do Problema . . . . . . . . . . . . . . . 59

2 Medida Comparativa de Desempenho . . . . . . . . . . . 62

3 Organização do Texto . . . . . . . . . . . . . . . . 64

Créditos ..................... 65

1 Ordenação Dinâmica $k$-partida como Jogo de Esconder $\quad 67$

2 Ordenação Dinâmica $k$-partida Seqüencial 71

2.1 Cotas para Algoritmos Determinísticos e Aleatorizados . . . . 72

2.2 Um Algoritmo Determinístico Otimamente Competitivo . . . 74

2.3 Ordenação Dinâmica 2-partida Seqüencial . . . . . . . . . 77

2.3.1 Cota para a Probabilidade da Competitividade . . . . 84

3 Ordenação Dinâmica $k$-partida Distribuída $\quad 87$

3.1 Cotas para a Competitividade de Algoritmos Determinísticos 87

3.2 Algoritmos Determinísticos . . . . . . . . . . . . . 88

3.2 .1 Modelo Preemptivo . . . . . . . . . . . . 88

3.2 .2 Modelo Não-Preemptivo . . . . . . . . . . . . . . 90

4 Um Jogo de Esconder Fatores de Grafo 93

4.1 Alguns Resultados sobre Fatores Esquerdos . . . . . . . . . . 94 
4.2 Estratégia de Evasão . . . . . . . . . . . . . . . 96

5 Comentários Finais 101

Índice de Definições . . . . . . . . . . . . . . . . . 106 



\section{Agradecimentos}

Não há como expressar em palavras minha gratidão para com o orientador deste trabalho, Yoshiharu Kohayakawa, a quem deve ser atribuída a maior parte dos méritos que este trabalho tenha. Yoshi foi um orientador na mais plena acepção da palavra. Do ponto de vista aprofissional um mestre, um exemplo e uma inspiração. Do ponto de vista pessoal, um apoio que esteve sempre além do que o papel de orientador acadêmico exigiria. O aprendizado e o amadurecimento que resultaram da oportunidade de convivência profissional com alguém desse calibre, profissional e humano, é sem dúvida o resultado mais valioso de todo o doutorado.

Este trabalho tem também uma dívida enorme para com Eduardo S. Laber, que o acompanhou e influenciou como cabe a um bom orientador. Efetivamente, não é senão por um acidente burocrático que seu nome não figura neste trabalho como co-orientador, como seria meu desejo e o de Yoshi, título a que faz jus sob qualquer ponto de vista.

Pelas mil e uma pequenas coisas que aconteceram no quotidiano do período em que fui aluno do Programa de Pós-Graduação do IME-USP, quero registrar aqui meu agradecimento aos professores do Departamento de Computação, muito em especial à Profa. Yoshiko Wakabayashi e ao Prof. Imre Simon, pessoas cuja convivência será para sempre uma referência. Quero também aqui fazer um especial agradecimento aos professores Júnior Barrera, João Eduardo Ferreira e ao aluno Gustavo Tadao, pela sua disponibilidade e boa vontade ante a idéia de implementar um experimento baseado nas idéias da segunda parte deste trabalho.

O mesmo agradecimento aos colegas com quem convivi mais diretamente nesse período, com especial menção a Jair Donadelli Jr., Fábio H. Viduani, Mario Leston e Aritanan "Pil" Gruber e uma menção muito especial aos funcionários da Comissão de Pós-Graduação e da Bibliteca Carlos Benjamin de Lyra, sempre atenciosos e competentes em suas funções. 
Também merece menção destacada o apoio dos colegas do Departamento de Informática da UFPR, responsáveis por criar as condições necessárias para a viabilidade deste trabalho. Menção muito especial vai aqui aos professores Roberto Hexsel, André Guedes e Marcos Sunyè, sempre a postos com a mais sorridente solicitude nos momentos em que alguma ajuda foi solicitada.

Como é do conhecimento dos que comigo conviveram mais proximamente a partir de meados de 2003, sofri um abalo de saúde sério que me obrigou a interromper temporariamente as atividades e por pouco não comprometeu o sucesso do projeto. Sou imensamente grato a todos os que conviveram comigo nesse período, cujo apoio e ajuda foram decisivos. Na impossibilidade de mencionar a todos, além dos já mencionados acima, fica aqui registrado meu mais sincero e profundo agradecimento a meus pais Paulo e Terezinha, a meus irmãos Cláudio e Sandra, a minhas tias Lourdes e Maria Francisca, e aos amigos Jean Lauand, Gabriel Perissé, João Sérgio Lauand, Luciano Penteado, Humberto Buzzi, Ronald Roesner, Jozef Skokan, Blanka Homolova, Ana Maria Brambilla, Erica Benvenutti, Cláudio Kassa, Gisele Santos, Vinícius Maciel, Marcelo Miki, Márcio Fernandes e Alessandra Carbonero.

Por fim, registro também meu agradecimento à CAPES, ao CNPq, à FAPESP e ao Centre de Recherches Mathematiques da Universidade de Montreal cujos programas de auxílio e financiamento permitiram a conclusão deste trabalho. 


\section{Introdução}

Neste trabalho estudamos dois problemas de busca que denominamos respectivamente Busca Ótima numa Ordem Parcial (BOOP) e Ordenação Dinâmica $k$-partida (ODk). O trabalho está dividido em duas partes, cada uma delas dedicada a um destes problemas.

O BOOP pode ser brevemente descrito como o problema de busca de um elemento num conjunto parcialmente ordenado. Posto de outra forma o BOOP pode ser visto como a generalização do tradicional problema de busca de um elemento em um conjunto totalmente ordenado.

O OD $k$, por sua vez, já é um problema mais específico, cuja motivação parte de uma necessidade operacional dos gerenciadores de bases de dados modernos, onde se deseja armazenar informação massiva (por exemplo imagens de alta resolução, seqüencias de informação genética etc) numa base de dados relacionais. Nesses casos, algumas das premissas operacionais assumidas pelos gerenciadores de bases de dados convencionais deixam de ser válidas. No caso que nos ocupa aqui, as premissas que deixam de ser válidas são duas. Primeiro, a de que o custo de avaliação de um predicado numa base de dados relacional é sempre o mesmo, independentemente da linha ou da coluna da relação a que se refira; segundo, que esse custo é praticamente desprezível se comparado ao custo total das operações envolvidas no processamento de uma consulta à base de dados. O OD $k$ corresponde à modelagem deste problema como um problema de busca num hipergrafo.

Em ambos os casos, lançamos mão do recurso de modelar o problema como um jogo entre dois jogadores, que denominamos genericamente Jogo de Esconder. Num Jogo de Esconder temos uma jogadora que denominaremos a ocultadora e o outro que denominaremos o procurador, cujas jogadas ao longo da evolução do jogo configuram respectivamente uma instância e um algoritmo para o problema em estudo. Esse tipo de abordagem nos permitirá formular e provar resultados de maneira clara, sem nos obrigar a desenvolver 
uma notação por demais carregada e poupar-nos-á também de tecnicidades que mais obscurecem que esclarecem as idéias centrais apresentadas.

Cada uma das partes do trabalho conta com um capítulo introdutório onde fazemos um resumo do conteúdo e enunciamos os principais resultados. 


\section{Preliminares}

Procuramos ater-nos à notação e nomenclatura usuais para algoritmos, grafos e ordens parciais

Para efeitos de referência, cada uma das duas partes do texto inicia com seu próprio capítulo de "Preliminares". O leitor familiarizado com a notação e nomenclatura usuais a respeito dos conceitos tratados nestes capítulos não encontrará dificuldade na leitura do corpo do trabalho ao omitir estes preliminares.

A próxima seção estabelece notação e convenções utilizadas ao longo de todo o texto. Embora em sua maioria sejam convenções também amplamente utilizadas na literatura da área, mesmo ao leitor familiarizado recomendamos ao menos uma breve leitura de seu conteúdo.

Finalmente, a seção subseqüente faz uma apresentação informal do conceito de uma classe de jogos como modelo computacional para o estudo de questões de complexidade computacional. Recomendamos sua leitura integral pois o resto do corpo do trabalho faz uso intensivo dos conceitos ali discutidos.

\section{Notação, Convenções e Algumas Definições}

O conjunto de subconjuntos de $n$ elementos de um dado conjunto $C$ será denotado por $\left(\begin{array}{l}C \\ n\end{array}\right)$ e o conjunto de todos os seus subconjuntos por $2^{C}$. Usamos lg para denotar $\log _{2}$ e log para o logaritmo natural.

Todas as análises de algoritmos neste trabalho dizem respeito ao tempo de execução do algoritmo em questão. Dando isso por subentendido, escrevemos simplesmente, por exemplo, "algoritmo polinomial" em vez de "algoritmo de tempo polinomial".

Dado um espaço de probabilidades $\Omega$ e um evento $E$ de $\Omega$, denotamos 
por $\overline{E_{\Omega}}$ o evento complementar de $E$ em $\Omega$, por $\mathbb{P}_{\Omega}(E)$ probabilidade de $E$ e por $\mathbb{E}_{\Omega}[E]$ a esperança de $E$. Sempre que o contexto permita fazêlo sem ambigüidade, omitimos o subscrito $\Omega$. Dado um conjunto finito $C$, denotamos por $\mathcal{U}(C)$ o espaço de probabilidades uniforme gerado por $C$. O suporte de $\Omega$ é o conjunto dos eventos unitários $E$ de $\Omega$ para os quais $\mathbb{P}_{\Omega}(E)>0$.

Uma árvore sobre um conjunto $C$ é denotada por uma tupla $T=\left(r(T), S_{1}(T), \ldots, S_{k}(T)\right)$, onde $r(T) \in C$ é a raiz de $T$, e $S_{i}(T)$, $1 \leq i \leq k$ são árvores sobre $C$, chamadas filhos de $T$. As árvores $S_{1}(T)$ e $S_{k}(T)$ às vezes chamadas de filho esquerdo e filho direito de $T$, respectivamente. A árvore vazia é denotada por $\Lambda$.

Um caminho em uma árvore $T$ é uma seqüência $\left(r_{1}, \ldots, r_{n}\right)$ onde $r_{1}=r(T)$ e, para cada $1<i \leq n$ temos que $r_{i}$ é raiz de algum dos filhos da árvore da qual $r_{i-1}$ é raiz. Um ramo de $T$ é um caminho maximal em $T$ a partir de sua raiz. Um ramo $R=\left(r_{1}, \ldots, r_{n}\right)$ onde, para cada $1<i \leq n, r_{i}$ é a raiz do filho direito da árvore da qual $r_{i-1}$ é raiz é chamado de ramo direito de $T$.

\section{Problemas de Busca Ótima e Jogos de Esconder}

Como primeira aproximação, podemos dizer que neste trabalho estudamos a complexidade média e de pior caso de algoritmos para problemas de busca. Para estudar a complexidade de pior caso, fazemos uso da formulação do problema em questão como um jogo entre dois jogadores.

Chamamos o tipo de jogos de que faremos uso no texto de "jogos de esconder" porque nesses jogos ambos os jogadores têm diante de si um conjunto de objetos e alguma informação estrutural a respeito deles. Um jogo de esconder inicia com a jogadora que chamaremos de ocultadora escolhendo um desses objetos. O outro jogador, que chamaremos de procurador, tem por objetivo descobrir qual o objeto escolhido pela ocultadora. Cada lance do jogo consiste numa jogada ou pergunta do procurador e na respectiva contra-jogada ou resposta da ocultadora.

Em clave um pouco mais formal, podemos definir um lance do jogo como um par $(p, r)$, onde $p$ é pergunta (do procurador) e $r$ é a resposta (da ocultadora). Os domínios de $p$ e $r$ são estabelecidos pela formulação do próprio jogo. Definimos uma realização do jogo como uma seqüência de lances do jogo e a duração dessa realização como o comprimento dessa seqüência. 
Um algoritmo que decide a próxima jogada de um jogador baseado no histórico do jogo será chamado uma estratégia; uma estratégia para o procurador será chamada uma estratégia de busca e uma estratégia para a ocultadora será chamada uma estratégia de evasão. Com isso queremos evitar a ambigüidade no uso da palavra "algoritmo", que reservamos para referir-nos a algoritmos para a solução do problema estudado, e não para os algoritmos (estratégias) que decidem as jogadas dos jogadores. Uma estratégia de busca será considerada tão boa quanto menor seja a duração da realização (mais longa possível) de um jogo em que o procurador a adota.

A idéia geral da tradução do problema de busca em jogo de esconder é que a estratégia de busca corresponde a um algoritmo para a resolução do respectivo problema de busca, enquanto que a estratégia de evasão corresponde a uma instância (ou, mais precisamente, classe de instâncias) do problema para a qual esse algoritmo tenha mau desempenho.

Para uma apresentação mais ampla e formal dessas idéias, referimos o leitor a Bollobás (1978, capítulo VIII). Será bastante para os nossos propósitos manter a equivalência entre problemas de busca e jogos de esconder em nível informal, apelando para a intuição do leitor. Para tanto, um exemplo concreto pode ser bastante esclarecedor. Considere o tradicional problema de busca em lista ordenada, formulado como segue.

\section{Problema: busca em lista ordenada}

Entrada: uma lista de inteiros $\left(l_{1}, \ldots, l_{n}\right)$ satisfazendo $l_{1} \leq \ldots \leq l_{n}$ e um inteiro $k$.

Saída: sim ou não, conforme exista ou não um $1 \leq i \leq n$ tal que $k=l_{i}$.

Este problema de busca pode ser formulado como um jogo de esconder da seguinte maneira. 


\section{Jogo: busca em lista ordenada $(\mathcal{J}(L))$}

Início: a ocultadora recebe uma lista de inteiros $L=\left(l_{1}, \ldots, l_{n}\right)$ satisfazendo

$l_{1} \leq \ldots \leq l_{n}$ e escolhe um inteiro $k$ qualquer; o procurador recebe somente o tamanho $n$ da lista.

Lance: cada pergunta é um número $p \in\{1, \ldots, n\}$; a resposta é $=,<$ ou $>$ conforme $l_{p}=k, l_{p}<k$ ou $l_{p}>k$, respectivamente.

Fim: O procurador encontra-se em condições de declarar se $k=l_{i}$ para algum $1 \leq i \leq n$.

Considere agora a seguinte estratégia de busca para o jogo da busca em lista ordenada.

$$
\begin{aligned}
& \text { Estratégia } \boldsymbol{B} \boldsymbol{B}(n) \\
& \begin{array}{l}
(i, f) \leftarrow(1, n) \\
\text { enquanto } i \leq f \\
\quad \text { pergunte } p=\lfloor(i+f) / 2\rfloor \\
\quad \text { se a resposta for }=\text {, o jogo termina com sim }\left(l_{p}=k\right) \\
\quad \text { se a resposta for }<\text {, faça } i \leftarrow p+1 \\
\quad \text { se a resposta for }>\text {, faça } f \leftarrow p-1 \\
\text { o jogo termina com não }\left(k \notin\left\{l_{1}, \ldots, l_{n}\right\}\right)
\end{array}
\end{aligned}
$$

O leitor reconhecerá facilmente na Estratégia $B B$ uma versão do tradicional algoritmo de busca binária. Interessa-nos ressaltar com esse exemplo que cada realização do jogo de busca em lista ordenada com a estratégia de busca $B B$ corresponde a uma execução do algoritmo de busca binária. A instância do problema (na verdade, classe de instâncias) a que corresponde essa execução, por sua vez, é determinada pela estratégia de evasão. Essas observações podem ser resumidas e generalizadas nos seguintes termos.

Seja $\mathcal{P}$ um problema de busca e seja $\mathcal{J}$ um correspondente jogo de esconder. Sejam ainda $\mathcal{E}$ uma estratégia de busca para o jogo $\mathcal{J}$ e seja $\mathcal{A}$ um algoritmo para o problema $\mathcal{P}$, correspondente à estratégia $\mathcal{E}$. 
Cada realização do jogo $\mathcal{J}$ com a estratégia de busca $\mathcal{E}$ corresponde a uma execução do algoritmo $\mathcal{A}$ sobre uma particular classe de instâncias de $\mathcal{P}$.

Se cada realização de um jogo segundo uma estratégia de busca é representada por uma seqüência (de lances), nada mais que natural, então, que representar todas as possíveis realizações do jogo para essa estratégia de busca por uma árvore cuja raiz representa a primeira pergunta, e cuja raiz de cada filho corresponde a cada uma das possíveis respostas. Chamamos uma árvore assim de árvore de busca dessa estratégia.

Voltemos ao nosso exemplo para uma ilustração. A árvore de busca da estratégia $B B$ é uma árvore $T=(p, T(<), T(>))$, sendo $p \in\{1, \ldots, n\}$ a primeira pergunta e $T(<)$, e $T(>)$ as árvores correspondentes às possíveis evoluções subseqüentes do jogo (cada uma correspondendo a cada possível resposta).

Nessa árvore, cada nó representa uma jogada do procurador, cada filho representa uma das possíveis contra-jogadas da ocultadora e cada ramo representa uma das possíveis realizações do jogo para a estratégia de busca $B B$. A altura da árvore é a duração do jogo no pior caso para a estratégia $B B$. Com isso temos uma medida da eficácia da estratégia $B B$ e, conseqüentemente, do algoritmo de busca binária. Podemos generalizar e resumir essas observações da seguinte forma.

Seja $\mathcal{P}$ um problema de busca e seja $\mathcal{J}$ um correspondente jogo de esconder. Sejam ainda $\mathcal{E}$ uma estratégia de busca para o jogo $\mathcal{J}$ e seja $\mathcal{A}$ um algoritmo para o problema $\mathcal{P}$, correspondente à Estratégia $\mathcal{E}$. Finalmente, seja $T$ a árvore de busca da Estratégia $\mathcal{E}$.

Cada ramo da árvore $T$ corresponde a uma execução do algoritmo $\mathcal{A}$ sobre uma particular classe de instâncias do problema $\mathcal{P}$. Conseqüentemente, a altura da árvore $T$ é uma medida do desempenho de pior caso da estratégia $\mathcal{E}$ e do algoritmo $\mathcal{A}$.

O leitor não terá dificuldade em observar que o que chamamos aqui de "a árvore de busca da estratégia $B B^{\prime \prime}$ coincide com a estrutura de dados conhecida como árvore de busca binária. Noutras palavras, podemos representar um algoritmo de busca sob a forma de uma árvore de busca. Nessa linha de raciocínio, podemos reformular o problema de busca em lista ordenada como segue. 


\section{Problema: busca em lista ordenada}

Entrada: uma lista de inteiros $L=\left(l_{1}, \ldots, l_{n}\right)$ satisfazendo $l_{1} \leq \ldots \leq l_{n}$ e um inteiro $k$.

Saída: uma árvore de busca para o jogo $\mathcal{J}(L)$.

A vantagem dessa reformulação é a de ser mais facilmente manuseável quando o que está em discussão é a comparação entre diversos algoritmos para o mesmo problema. Por exemplo, considere o problema de determinar uma estratégia ótima de busca numa lista ordenada.

\section{Problema: busca ótima em lista ordenada}

Entrada: uma lista de inteiros $L=\left(l_{1}, \ldots, l_{n}\right)$ satisfazendo $l_{1} \leq \ldots \leq l_{n}$ e um inteiro $k$.

Saída: uma árvore de busca de altura mínima o jogo $\mathcal{J}(L)$.

Chamamos a atenção para a diferença entre os problemas de busca em lista ordenada e busca ótima em lista ordenada. Para o primeiro, qualquer árvore de busca (ou, correspondentemente, qualquer estratégia de busca) representa uma solução; no segundo, somente uma árvore de busca de altura mínima (correspondentemente, uma estratégia de busca ótima) representa uma solução.

Resumindo e generalizando estas últimas observações, podemos estabelecer o seguinte.

Seja $\mathcal{J}$ um jogo de esconder. O problema de busca ótima associado a $\mathcal{J}$ é o problema de determinar uma estratégia de busca ótima para o jogo $\mathcal{J}$, ou, noutras palavras, o problema de determinar uma estratégia de busca cuja respectiva árvore de busca tem altura mínima.

No restante do texto utilizamos a correspondência exposta acima entre problemas de busca e jogos de esconder bem como entre algoritmos de busca e estratégias de busca. Dessa forma, com freqüência enunciamos resultados a respeito dos algoritmos em discussão em termos da altura da árvore de busca da estratégia de busca correspondente. Encerramos esta introdução com a seguinte cota mínima para problemas de busca ótima. 
Lema 1. Seja $\mathcal{P}$ um problema de busca ótima e seja $\mathcal{J}$ um seu correspondente jogo de esconder. Numa instância do jogo $\mathcal{J}$ onde o procurador tem $n$ possiveis perguntas e na qual a ocultadora pode dar até $k$ respostas diferentes a cada uma delas (além da resposta $=$ ), a duração de uma realização do jogo $\mathcal{J}$, qualquer que seja a estratégia de busca, é pelo menos $\left\lfloor\log _{k} n\right\rfloor+1$.

Demonstração. Seja $\mathcal{E}$ uma estratégia de busca para o jogo $\mathcal{J}$ e seja $T$ sua árvore de busca. A árvore $T$ tem pelo menos $n$ nós e cada nó tem no máximo $k$ filhos. Assim, $T$ não pode ter altura menor que $\left\lfloor\log _{k} n\right\rfloor+1$. 
Parte I

\section{Busca Ótima em Ordens Parciais}





\section{Preliminares}

\section{Grafos}

Um grafo é um par $G=(V(G), E(G))$, onde $V(G)$ é um conjunto e $E(G) \subseteq\left(\begin{array}{c}V(G) \\ 2\end{array}\right)$. Cada elemento de $V(G)$ é chamado um vértice de $G$, e cada elemento de $E(G)$ é chamado uma aresta de $G$. Dizemos que $v$ é uma ponta de $e$ ou, equivalentemente, que $e$ incide em $v$ quando $v \in e$.

A vizinhança de um vértice $v \in V(G)$ em $G$ é o conjunto $\Gamma_{G}(v)=\{u \in V(G):\{u, v\} \in E(G)\}$. A idéia de vizinhança estende-se naturalmente para conjuntos de vértices, de modo que se $X \subseteq V(G)$, a vizinhança de $X$ em $G$ é $\Gamma_{G}(X)=\bigcup_{v \in X} \Gamma_{G}(v)$. O grau de um vértice $v$ em $G$ é o número de arestas incidentes em $v$, isto é, $\operatorname{deg}_{G}(v)=|\{e \in E(G): v \in e\}|$.

Dado um grafo $G$ e um conjunto de vértices $X \subseteq V(G)$, o subgrafo de $G$ induzido por $X$ é o grafo $G[X]=(X,\{e \in E(G): e \subseteq X\})$.

Um grafo dirigido é um par $G=(V(G), A(G))$ onde $V(G)$ é um conjunto e $A(G) \subseteq V(G) \times V(G)$. Cada elemento de $V(G)$ é chamado um vértice de $G$, e cada elemento de $A(G)$ é chamado um arco de $G$.

Omitimos o subscrito $G$ da notação sempre que o contexto permita fazêlo sem ambigüidade.

\section{Ordens}

Uma ordem (ou ordem parcial), é um par $\left(P, \prec_{P}\right)$, onde $P$ é um conjunto e $\prec_{P}$ é uma relação binária anti-reflexiva, anti-simétrica, e transitiva sobre $P$. Com certo abuso da notação, aderimos à prática de referir-nos à ordem $\left(P, \prec_{P}\right)$ por $P$.

Dados $x, y \in P$, dizemos que $x$ é menor que y em $P$ ou, equivalentemente, que $y$ é maior que $x$ em $P$ se $(x, y) \in \prec_{P}$, fato que denotamos por 
$x \prec_{P} y$ ou $y \succ_{P} x$, indistintamente. Denotamos a situação em que $x \prec_{P} y$ ou $x=y$ por $x \preceq_{P} y$ ou $y \succeq_{P} x$. O caso em que $x \neq y$ e $(x, y) \notin \prec_{P}$, é denotado $x \npreceq_{P} y$. Um elemento maximal de uma ordem é um elemento que não é menor que nenhum outro elemento da ordem. Se $x, y, z \in P$ são tais que $x \prec_{P} y$ e $y \prec_{P} z$, dizemos que $y$ está entre $x$ e $z$ em $P$. Dizemos que $y$ cobre $x$ em $P$ se $x \prec P$ e não existe nenhum elemento de $P$ entre $x$ e $y$, o que se denota $x \prec y$.

Dizemos que $x$ e $y$ são comparáveis em uma ordem $P$ quando $x \prec_{P} y$ ou $x \succ_{P} y$, o que se denota $x \sim_{P} y$. Se $x$ e $y$ não são comparáveis, dizemos que são incomparáveis, o que se denota $x \nsim_{P} y$. Um posto de uma ordem é um elemento comparável a todos os outros elementos da ordem. Uma cadeia de uma ordem é um conjunto de elementos dois a dois comparáveis e uma anti-cadeia é um conjunto de elementos dois a dois incomparáveis. Uma ordem $P$ é total se $P$ é uma cadeia.

A altura de uma ordem $P$, denotada $h(P)$, é a cardinalidade de uma cadeia máxima em $P$ e a largura de $P$, denotada $w(P)$, é a cardinalidade de uma anti-cadeia máxima de $P$.

Dado um subconjunto $X$ de uma ordem $P$ denotamos por $P[X]$ a ordem induzida em $X$ por $P$, isto é, a ordem $(X, \prec P[X])$ onde $\prec_{P[X]}=\prec_{P} \cap(X \times X)$. A ordem dual de uma ordem $P$, denotada $P^{*}$, é a ordem $\left(P, \prec_{P}^{-1}\right)$, onde $\prec_{P}^{-1}=\left\{(y, x) \in P \times P: x \prec_{P} y\right\}$.

Um ideal de uma ordem $P$ é um conjunto $I \subseteq P$ no qual, para todo $i \in I$ vale que, se $s \prec_{P} i$, então $s \in I$. Dado $s \in P$, o ideal de $P$ gerado por $s$ é o ideal minimal $I$ de $P$ que contém $s$, isto é $I_{P}(s)=\left\{x \in P: x \preceq_{P} s\right\}$. O conceito estende-se naturalmente a um conjunto $X \subseteq P$, de forma que o ideal de $P$ gerado por $X$ é $I_{P}(X)=\bigcup_{x \in X} I_{P}(x)$. Definimos ainda $I_{P}^{-}(X)=I_{P}(X)-X$.

Um filtro de $P$ é um ideal de $P^{*}$. Dados $s \in P$ e $X \subseteq P$, ficam definidos naturalmente os conceitos de filtro de $P$ gerado por $s$ e filtro de $P$ gerado por $X$, denotados respectivamente por $F_{P}(s)$ e $F_{P}(X)$ e também $F_{P}^{-}(X)$.

$\mathrm{O}$ conjunto dos postos de uma ordem $P$ é denotado por $\pi(P)$. Para cada $1 \leq k \leq|\pi(P)|$, denotamos por $\pi_{k}(P)$ o $k$-ésimo posto de $P$, de maneira que $\pi_{k}(P) \prec_{P} \pi_{k+1}(P)$ para cada $1 \leq k<|\pi(P)|$.

Um segmento de $P$ é a ordem induzida por $P$ sobre os elementos entre dois postos consecutivos de $P$. Para cada $1 \leq k<|\pi(P)|$ denotamos por $S_{k}(P)$ o segmento compreendido entre $\pi_{k}(P)$ e $\pi_{k+1}(P)$, isto é, $S_{k}(P)=P\left[F^{-}\left(\pi_{k}(P)\right) \cap I^{-}\left(\pi_{k+1}(P)\right)\right]$. Além disso, convencionamos chamar de 0-ésimo segmento de $P$ à ordem $S_{0}(P)=P\left[I^{-}\left(\pi_{1}(P)\right)\right]$ e de 
$|\pi(P)|$-ésimo segmento de $P$ à ordem $S_{\pi(P)}(P)=P\left[F^{-}\left(\pi_{|\pi(P)|}(P)\right)\right]$. Note que segmentos podem ser vazios.

O diagrama de Hasse de uma ordem $P$ é o grafo dirigido $\mathcal{H}(P)=(P,\{(x, y) \in P \times P: y \prec x\})$. Ao desenhar o Diagrama de Hasse de uma ordem, desenha-se os arcos do grafo sem indicar sua orientação explicitamente, assumindo por convenção que estão sempre orientados de cima para baixo.

\section{Modelos Probabilísticos}

Um modelo probabilístico para uma certa classe de objetos (por exemplo, grafos) é uma família de espaços de probabilidade $\left\{\Omega_{n}: n>0\right\}$, onde, para cada $n>0$, os eventos elementares de $\Omega_{n}$ são os objetos dessa classe. Dizemos que um evento $E_{n}$ de $\Omega_{n}$ é um evento assintoticamente quase certo em $\Omega_{n}$ quando

$$
\lim _{n \rightarrow \infty} \mathbb{P}_{\Omega_{n}}\left(E_{n}\right)=1
$$

e dizemos que $E_{n}$ é um evento assintoticamente raro se $\overline{E_{n}}$ é um evento assintoticamente quase certo. Com a costumeira liberdade de expressão desse contexto, usamos expressões como "quase certo", "quase todo", "quase sempre" etc para designar eventos assintoticamente quase certos e expressões como "raro", "quase nenhum", "quase nunca" etc para designar eventos assintoticamente raros. Do mesmo modo, dizemos que uma determinada propriedade é uma propriedade típica em $\Omega_{n}$ se o evento de apresentar essa propriedade é quase certo em $\Omega_{n}$, e dizemos que é uma propriedade rara se o evento de apresentá-la é raro.

A título de ilustração, considere o modelo probabilístico para grafos não dirigidos dado por $\Omega_{n}=\mathcal{U}\left(\mathcal{G}_{n}\right)$, onde $\mathcal{G}_{n}$ denota o conjunto de todos os grafos não dirigidos com $V(G)=\{1, \ldots, n\}$. Diríamos então que (veja, por exemplo, Janson, Luczak, and Rucinski (2000)), "quase todo grafo em $\Omega_{n}$ é conexo", ou equivalentemente, que "o grafo típico de $\Omega_{n}$ é conexo" ou ainda que "conexidade é uma propriedade típica (dos grafos) em $\Omega_{n}$ ". 


\section{Introdução}

Um problema fundamental em Ciência da Computação é o de representar um conjunto cujo conteúdo varia, de maneira que seja possível efetuar operações de busca eficientemente (Knuth, 1998). Nesse contexto, uma das presunções mais comumente feitas a respeito dos elementos de um tal conjunto, é a de que são totalmente ordenados. Estudamos aqui uma variante desse problema na qual os elementos do conjunto em questão são apenas parcialmente ordenados.

Como acontece no estudo de algoritmos de busca para conjuntos totalmente ordenados, nosso critério de comparação entre diferentes algoritmos será o número máximo de comparações necessário para realizar qualquer busca nesse conjunto, ou, noutras palavras, o número de comparações feito pelo algoritmo numa análise de pior caso.

O problema estudado nesta parte do trabalho pode ser formulado como o problema de determinar uma estratégia de busca ótima para o seguinte jogo de esconder.

Jogo: busca em ordens parciais $(\mathcal{J}(P, S))$

Início: ambos jogadores recebem uma ordem $P$ e um conjunto $S \subseteq P$; a ocultadora escolhe um elemento $u \in P$

Lance: cada pergunta é um elemento $s \in S$; a resposta é $=, \prec$ ou $\npreceq$, conforme $s=u, s \prec_{P} u$ ou $s \varliminf_{P} u$, respectivamente

Fim: o procurador encontra-se em condições de declarar se $u \in S$

O correspondente problema de busca ótima pode ser formulado como segue. 


\section{Problema: busca ótima em ordens parciais}

Entrada: uma ordem $P$ e um conjunto $S \subseteq P$.

Saída: uma árvore de busca de altura mínima para o jogo $\mathcal{J}(P, S)$.

O problema é definido a partir de uma ordem $P$ e um conjunto $S \subseteq P$. A razão de manter ambas as ordens, $P$ e $P[S]$, em vez de referir-nos somente a $P[S]$, vem da motivação original do problema: com essa definição ligeiramente menos simples, incluímos o caso de buscas frustradas. Como o leitor perceberá, o papel da ordem $P$ será praticamente "decorativo".

Assumimos que a entrada do problema consiste num grafo dirigido em representação do diagrama de Hasse da ordem $P[S]$ e convencionamos designar uma "árvore de busca para o jogo $\mathcal{J}(P, S)$ " simplesmente por árvore para $P[S]$. Dando por subentendidas estas convenções, podemos formular de maneira mais concisa o problema nos seguintes termos.

\section{Problema: busca ótima em ordens parciais (BOOP)}

Entrada: uma ordem $P$.

Saída: uma árvore de altura mínima para $P$.

Chamamos a atenção do leitor para o fato de que o problema de busca ótima em lista ordenada tal como formulado ao final da Seção 2 corresponde exatamente à restrição do BOOP às instâncias onde a ordem dada é total.

\section{Organização do Texto}

Provamos inicialmente, no Capítulo 1 (Teorema 1.8), que o problema é $\mathcal{N}$ P-difícil, respondendo à principal questão deixada em aberto por Ben-Asher, Farchi, and Newman (1999), trabalho que foi o motivador inicial do presente estudo. Provamos também ao final deste capítulo (Corolário 1.9), como conseqüência imediata do Lema 1 (página 9), que toda árvore de busca para uma ordem de $n$ elementos tem altura pelo menos $\lg n$. A partir daí, concentramos a atenção na formulação de algoritmos polinomiais para o BOOP que apresentam bom desempenho de caso médio.

Falar em "bom desempenho de caso médio" implica falar numa distribuição de probabilidades subjacente ao conjunto de instâncias do problema, 
ou seja, um modelo probabilístico para ordens parciais. Ao contrário do que acontece com modelos probabilísticos para outros objetos combinatórios, no caso de ordens existem diversos modelos de interesse que não são equivalentes entre si. Mais ainda, nenhum deles se impõe como o "mais natural" ou "o mais conveniente". Em se tratando de ordens aleatórias, portanto, é preciso decidir a respeito de qual modelo se trata. Concentramos nosso estudo sobre dois modelos que talvez se possa chamar de os "mais clássicos": o modelo grafo-aleatório e o modelo uniforme. O Capítulo 2 dedica-se a descrever estes modelos e enunciar alguns resultados fundamentais a respeito de cada um deles.

O Capítulo 3 apresenta um algoritmo para o BOOP que computa em tempo polinomial uma árvore de altura máxima $(1+o(1)) \lg n$ para uma ordem grafo-aleatória típica de $n$ elementos. À luz do Corolário 1.9 (página 27), esse algoritmo resulta numa $(1+o(1))$-aproximação para uma instância típica do problema no modelo grafo-aleatório.

No Capítulo 4 estudamos algoritmos para o modelo uniforme. Mostramos inicialmente que qualquer árvore para uma ordem típica de $n$ elementos tem altura $(1+o(1)) n / 4$. Noutras palavras, mostramos que não existe estratégia de busca para o jogo de busca em ordens parciais cujo desempenho típico seja assintoticamente melhor que a estratégia de busca seqüencial, que ignora a informação dada pela ordem $P$ e compara o elemento procurado com cada um dos elementos de $S$ até encontrá-lo ou esgotar o conjunto. Isso acontece porque o problema, tal como formulado originalmente por Ben-Asher et al. (1999), é de certa forma "assimétrico". Nessa formulação, dada uma ordem $P$ e um elemento $u \in P$ pelo qual se procura, é permitido ao procurador fazer perguntas do tipo $x \prec_{P} u$ mas não perguntas do tipo $x \succ_{P} u$.

Em vista disso, passamos a considerar a natural variação do problema onde perguntas do tipo $x \succ_{P} u$ também são permitidas no jogo. Mostramos que nessa variação do problema toda árvore para uma ordem de $n$ elementos tem altura pelo menos $\log _{3} n$ e apresentamos um algoritmo também simples (embora talvez um pouco menos natural à primeira vista), que computa uma árvore de altura máxima $(6.33 \ldots+o(1)) \log _{3} n$ para a ordem típica de $n$ elementos. Portanto, esse algoritmo representa uma 6.34-aproximação para a instância típica do problema no modelo uniforme.

Tratamos brevemente, no Capítulo 5, de um problema de busca ótima correlato ao BOOP, proposto por Linial and Saks (1985a), que designamos por busca ótima com informação parcial (BOIP). Mostramos que, apesar de tratar-se de diferentes problemas, os resultados relativos ao BOOP no modelo 
grafo-aleatório podem ser adaptados ao BOIP.

Finalmente, no Capítulo 6 resenhamos alguns resultados relativos a busca em conjuntos parcialmente ordenados e fazemos alguns comentários adicionais.

\section{Créditos}

Este trabalho foi realizado em conjunto com Jair Donadelli (Departamento de Informática da Universidade Federal do Paraná), Yoshiharu Kohayakawa (Departamento de Ciência da Computação do IME-USP) e Eduardo Laber (Departamento de Informática da PUC-RJ).

Uma versão preliminar deste trabalho foi apresentada como "Resumo Estendido" na edição de 2002 do Latin American Theoretical INformatics (LATIN 2002) (Carmo, Donadelli, Kohayakawa, and Laber, 2002). A versão final foi publicada em Carmo, Donadelli, Kohayakawa, and Laber (2004a). 


\section{Capítulo 1}

\section{Complexidade Computacional}

Neste capítulo provamos que o BOOP é $\mathcal{N P}$-difícil respondendo à principal questão deixada em aberto por Ben-Asher et al. (1999), motivador original do presente estudo. Ao final, provamos também que toda árvore de busca para uma ordem de $n$ elementos tem altura pelo menos $\lg n$.

Reduzimos o problema de cobertura exata por 3-conjuntos, que é $\mathcal{N}$ P-completo (Papadimitriou, 1994, p. 201), a uma versão em problema de decisão do BOOP. Principiamos por formular precisamente os problemas envolvidos na redução.

Problema de Decisão: cobertura exata por 3-Conjuntos (CE3C)

Entrada: um par $(X, \Delta)$, onde $X$ é um conjunto finito cujo tamanho é múltiplo de 3 e $\Delta \subseteq\left(\begin{array}{l}X \\ 3\end{array}\right)$.

Pergunta: existe um conjunto $\Gamma \subseteq \Delta$ de tamanho $|X| / 3$ tal que

$$
\bigcup_{\gamma \in \Gamma} \gamma=X ?
$$


A versão em problema de decisão do BOOP que consideramos é a seguinte.

\section{Problema de Decisão: busca ótima em ordens parciais (BOOPd)}

Entrada: um par $(P, k)$, onde $P$ é uma ordem e $k$ é um inteiro.

Pergunta: existe uma árvore de busca com altura no máximo $k$ para $P$ ?

Dada uma instância $(X, \Delta)$ para o CE3C, definimos $\left(P_{X, \Delta}, k(X, \Delta)\right)$, uma instância para o BOOPd (veja a Figura 1.1 para uma representação esquemática), fazendo $k(X, \Delta)=|X| / 3+2|\Delta|+3$. Para definir a ordem $P_{X, \Delta}$, tomamos três novos elementos $\bar{x}_{1}, \bar{x}_{2}, \bar{x}_{3} \notin X$ e a partir deles definimos os conjuntos

$$
\begin{aligned}
\bar{X} & =\left\{\bar{x}_{1}, \bar{x}_{2}, \bar{x}_{3}\right\} \\
\bar{Q} & =(X \cup \bar{X}) \times\{1, \ldots, 2|\Delta|\} .
\end{aligned}
$$

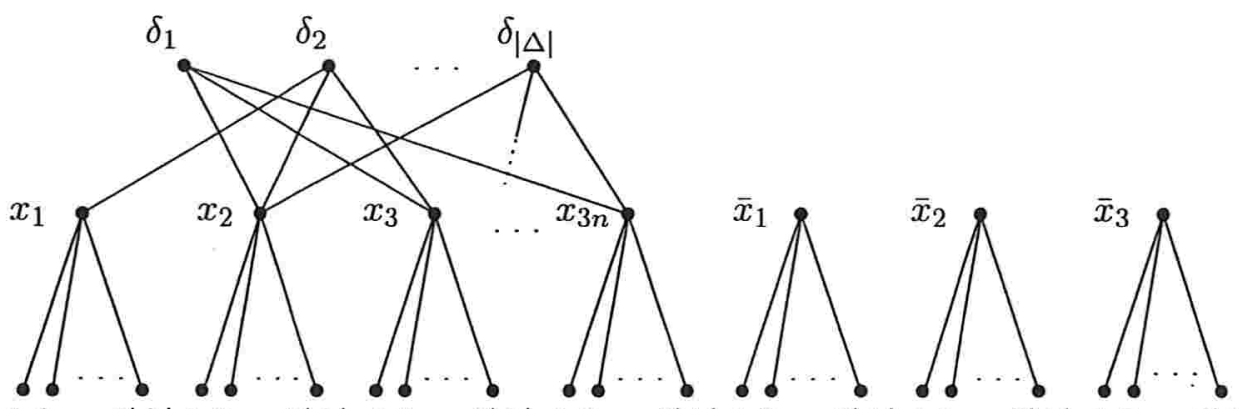

$12 \cdots 2|\Delta| 12 \cdots 2|\Delta| 12 \cdots 2|\Delta| 12 \cdots 2|\Delta| 12 \cdots 2|\Delta| 12 \cdots 2|\Delta| 12 \cdots 2|\Delta|$

Figura 1.1: Representação esquemática da ordem $P_{X, \Delta}$

Os elementos da ordem $P_{X, \Delta}$ são dados por $P_{X, \Delta}=\Delta \cup X \cup \bar{X} \cup \bar{Q}$, e a relação $\prec_{P_{X, \Delta}}$ é dada por

1. $x \prec_{P_{X, \Delta}} \delta$, para todo $x \in \delta$ de cada $\delta \in \Delta$,

2. $(x, j) \prec_{P_{X, \Delta}} x$ para todo $x \in X \cup \bar{X}$ e todo $j \in\{1, \ldots, 2|\Delta|\}$.

Mais concisamente, a relação $\prec_{P_{X, \Delta}}$ é o fecho transitivo de

$\prec P_{X, \Delta}=\{(x, \delta): x \in X$ e $\delta \in \Delta\} \cup\{((x, j), x): x \in X \cup \bar{X}$ e $1 \leq j \leq 2|\Delta|\}$ 
Para provar que existe uma cobertura exata para $(X, \Delta)$ se e somente se existe uma árvore de altura no máximo $k(X, \Delta)$ para $P_{X, \Delta}$, principiamos por dois resultados auxiliares. Antes, porém, a fim de descarregar a notação, pelo resto deste capítulo adotamos as seguintes convenções

$$
\begin{aligned}
k & =k(X, \Delta), \\
P & =P_{X, \Delta}, \\
\prec & =\prec_{P_{X, \Delta}} .
\end{aligned}
$$

Lema 1.1. A ordem $P\left[I^{-}(\delta)\right]$ tem uma árvore de altura $3+2|\Delta|$ para todo $\delta \in \Delta$.

Demonstração. Seja $\delta \in \Delta$ e considere a estratégia de busca $\mathcal{E}_{1}$ abaixo para o jogo $\mathcal{J}$ (veja a Figura 1.2).

\section{Estratégia $\mathcal{E}_{1}(P, S)$}

pergunte $x$ para cada $x \in \delta$

se a resposta for $=$, o jogo termina $\left(u=x \in I^{-}(\delta)=S\right)$

se a resposta for $\prec$

pergunte $y$ para cada $y \in I(x)$ até que a resposta seja $=, 0$ que termina o jogo $\left(u=y \in I(x) \subseteq I^{-}(\delta)=S\right)$

o jogo termina $\left(u \notin I^{-}(\delta)=S\right)$

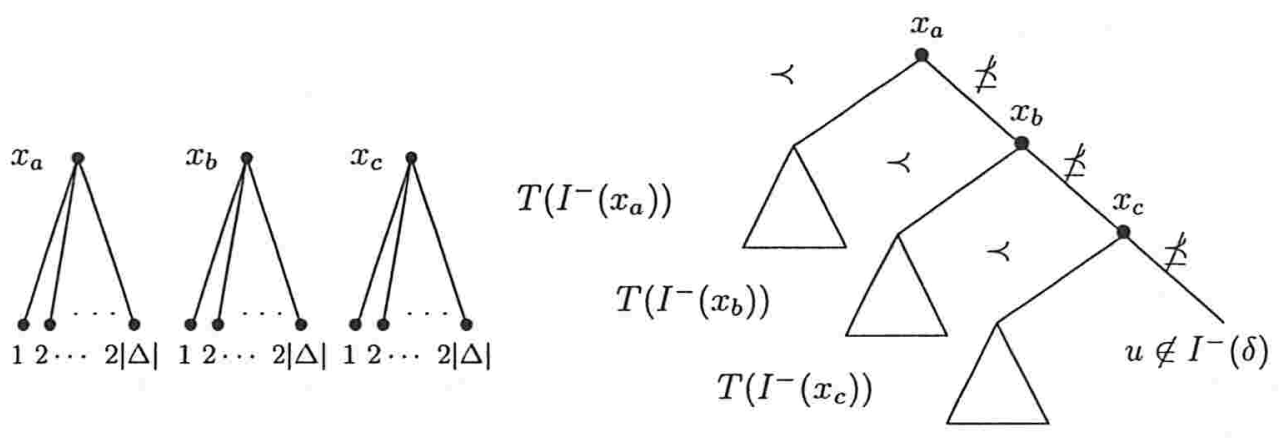

Figura 1.2: A ordem $P\left[I^{-}(\delta)\right]$ e a árvore da estratégia $\mathcal{E}_{1}$ no jogo $\mathcal{J}\left(P, I^{-}(\delta)\right)$

$\mathrm{O}$ caráter exaustivo da Estratégia $\mathcal{E}_{1}$ não deixa dúvidas quanto à sua correção. Também é imediato que a duração do jogo $\mathcal{J}\left(P, I^{-}(\delta)\right)$ com essa 
estratégia de busca não pode ser maior que

$$
|\delta|+\max \left\{\left|I^{-}(x)\right|: x \in \delta\right\}=3+2|\Delta| .
$$

A ordem $P\left[I^{-}(\delta)\right]$, portanto, tem uma árvore de altura $3+2|\Delta|$.

Lema 1.2. Sejam $S \subseteq P$ e $x \in X \cup \bar{X}$ dados. Se $I(x) \subseteq S$, então toda árvore para $P[S]$ tem altura pelo menos $2|\Delta|+1$.

Demonstração. Considere a estratégia de evasão $\mathcal{E}_{3}$ abaixo para o jogo $\mathcal{J}$, que consiste em responder $=$ somente à última pergunta a um elemento de $I(x)$.

Estratégia $\mathcal{E}_{3}(P, S)$

$X \leftarrow \emptyset$

a cada pergunta $p$

$$
\begin{aligned}
& \text { se } x \preceq p \text {, responda } \prec \\
& \text { se } p \in I^{-}(x) \\
& \quad X \leftarrow X \cup\{p\} \\
& \quad \text { se } X=I(x), \text { responda }= \\
& \quad \text { caso contrário, responda } \npreceq \\
& \text { responda } \npreceq
\end{aligned}
$$

É imediato da formulação da Estratégia $\mathcal{E}_{3}$ que a duração de um jogo $\mathcal{J}(P, S) \operatorname{com} I(x) \subseteq S$ é pelo menos $|I(x)|=2|\Delta|+1$.

Portanto, toda árvore de busca para $P[S]$ com $I(x) \subseteq S$ tem altura pelo menos $2|\Delta|+1$.

A redução propriamente dita fica estabelecida com os dois próximos resultados.

Teorema 1.3. Se $(X, \Delta)$ tem cobertura exata, então existe árvore para $P$ de altura no máximo $k$.

Demonstração. Seja $C$ uma cobertura exata para $X$, e considere a estratégia de busca $\mathcal{E}_{2}$ abaixo para o jogo $\mathcal{J}$. 


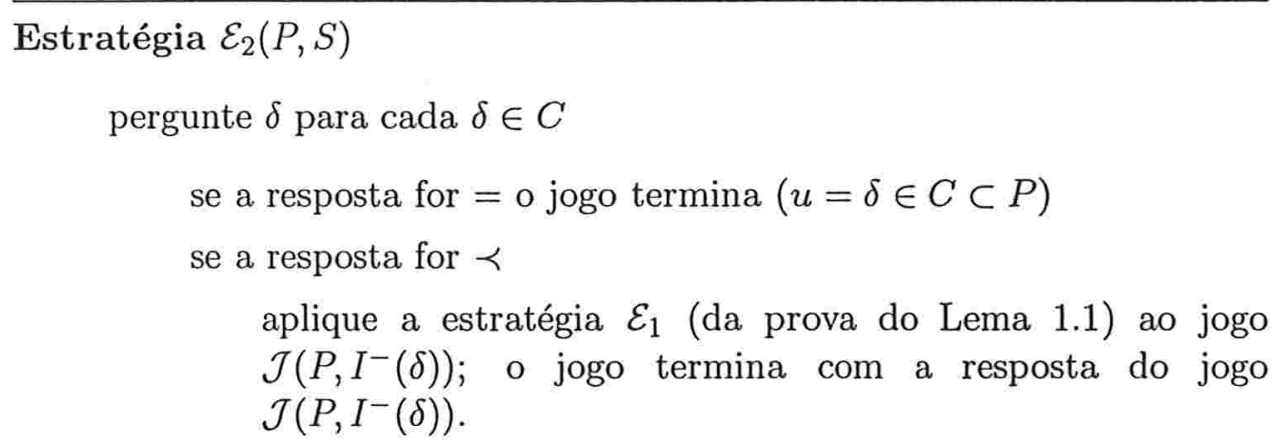

pergunte $\bar{x}$ para cada $\bar{x} \in \bar{X}$

se a resposta for $=0$ jogo termina $(u=\bar{x} \in \bar{X} \subset P)$

se a resposta for $\prec$, pergunte $y$ para cada $y \in I^{-}(\bar{x})$

se a resposta for $=$, o jogo termina $\left(u=y \in I^{-}(\bar{x}) \subset P\right)$

pergunte $\delta$ para cada $\delta \in \Delta-C$

se a resposta for $=$, o jogo termina $(u=\delta \in \Delta-C \subset P)$

o jogo termina $(u \notin I(\Delta \cup \bar{X}))$

$\mathrm{O}$ caráter exaustivo da Estratégia $\mathcal{E}_{2}$ não deixa dúvidas quanto à sua correção. Também é imediato que a duração do jogo $\mathcal{J}(P, P)$ com essa estratégia de busca não pode ser maior que

$$
\begin{aligned}
\max \{|C|+\max \{j(\delta): \delta \in C\}, & \\
|C|+|\bar{X}|+\max \left\{\left|I^{-}(\bar{x})\right|: \bar{x} \in \bar{X}\right\}, & |C|+|\bar{X}|+|\Delta-C|\}
\end{aligned}
$$

lances, onde $j(\delta)$ denota a duração do jogo $\mathcal{J}(P, I(\delta))$ com a estratégia de busca $\mathcal{E}_{1}$ (da prova do Lema 1.1).

De acordo com o Lema 1.1 temos $\max \{j(\delta): \delta \in C\}=3+2|\Delta|$. Por hipótese temos $|C|=|X| / 3$ e por construção temos

$$
\max \left\{\left|I^{-}(x)\right|: x \in\left\{\bar{x}_{1}, \bar{x}_{2}, \bar{x}_{3}\right\}\right\}=2|\Delta|
$$

e $|\bar{X}|=3$ Assim a duração do jogo $\mathcal{J}(P, P)$ com a estratégia de busca $\mathcal{E}_{2}$ é no máximo

$$
\max \{|X| / 3+3+2|\Delta|,|X| / 3+3+2|\Delta|,|\Delta|+3\}=|X| / 3+3+2|\Delta|=k .
$$


Portanto, existe uma árvore de altura no máximo $k=|X| / 3+2|\Delta|+3$ para a ordem $P=P_{X, \Delta}$.

Teorema 1.4. Se existe árvore para $P=P_{X, \Delta}$ de altura até $k=k(X, \Delta)=|X| / 3+2|\Delta|+3$, então existe uma cobertura exata para $(X, \Delta)$.

Demonstração. Seja $T$ uma árvore ótima para $P$, faça $n=|X| / 3$ e suponha

$$
h(T) \leq n+2|\Delta|+3 .
$$

Fato 1.5. O ramo direito de $T$ tem comprimento pelo menos $n+3$.

Demonstração. Seja $R$ o ramo direito de $T$. Cada um dos $3 n+3$ elementos de $X \cup \bar{X}$ está em $R$ ou é menor do que algum elemento em $R$. Entretanto, um elemento $s \in P$ tem em seu ideal, ou até 3 elementos de $X$ ou então no máximo 1 elemento de $\bar{X}$, isto é, $|I(s) \cap X| \leq 3$ e $I(s) \cap \bar{X}=\emptyset$, ou $I(s) \cap X=\emptyset$ e $|I(s) \cap \bar{X}| \leq 1$. Portanto, $R$ tem de ter comprimento pelo menos $n+3$.

Seja $R=\left(a_{1}, \ldots, a_{m}\right)$ o ramo direito de $T$ e seja $A=\left\{a_{1}, \ldots, a_{n+3}\right\}$, com $n+3 \leq m$.

Fato 1.6. Se $x \in X \cup \bar{X}$ não é comparável a nenhum elemento de $A$, então $I(x)$ está contido no filho direito de $a_{n+3}$.

Demonstração. Seja $y \in I(x)$. Como $x$ não é comparável a nenhum elemento de $A$, não podemos ter $y \in A$.

Também não pode ser o caso de que $y$ esteja no filho esquerdo de $a$ para algum $a \in A$. Nesse caso teríamos $y \prec a$, e como $y \in I(x)$ e $x \in X \cup \bar{X}$, teríamos $a=x$, ou $a=\delta$ para algum $\delta \in \Delta$ com $x \prec \delta$. Em qualquer caso, teríamos $x$ comparável a algum elemento de $A$, contradizendo a hipótese.

Como $y$ não está em $A$ nem no filho esquerdo de nenhum elemento de $A$, resta concluir que $y$ está no filho direito de $a_{n+3}$.

Fato 1.7. Todo elemento de $X \cup \bar{X}$ é comparável a algum elemento de $A$.

Demonstração. Suponha um elemento $x \in X \cup \bar{X}$ que não é comparável a nenhum elemento de $A$.

O fato de que $I(x)$ está contido no filho direito de $a_{n+3}$ e o Lema 1.2 implicam que $h(T) \geq n+2|\Delta|+4$, contra o assumido em (1.1). 
Como todo elemento de $X \cup \bar{X}$ é comparável a algum elemento de $A$, em particular $|I(\bar{X}) \cap A| \geq 3$, e daí $|A-I(\bar{X})| \leq n$. Como todo elemento de $X$ é comparável a algum elemento de $A$ e $|X|=3 n$, conclui-se facilmente que $A-I(\bar{X})$ tem de ser uma cobertura exata para $X$.

Corolário 1.8. O BOOP é NPP-difícil.

Demonstração. O BOOPd é uma versão em problema de decisão do BOOP. Claramente, o BOOPd está em $\mathcal{N P}$. O CE3C é um problema $\mathcal{N} \mathcal{P}$-completo (Papadimitriou, 1994, p. 201). Os Lemas 1.3 e 1.4 estabelecem uma redução claramente polinomial do $\mathrm{CE} 3 \mathrm{C}$ ao BOOPd. Isso prova que o BOOPd também é $\mathcal{N} \mathcal{P}$-completo e que, portanto, o BOOP é $\mathcal{N} \mathcal{P}$-difícil.

Provamos a seguir, um resultado de cota mínima para o BOOP, decorrência imediata do Lema 1 (página 9).

Corolário 1.9. Qualquer árvore de busca para uma ordem de $n$ elementos tem altura pelo menos $\lfloor\lg n+1\rfloor$.

Demonstração. Uma árvore de busca para uma ordem $P$ é uma árvore de busca para alguma estratégia de busca no jogo $\mathcal{J}(P, P)$. Nesse jogo, o procurador tem $|P|=n$ perguntas possíveis e a ocultadora tem sempre 2 respostas possíveis a cada lance, além da resposta $=$. Por decorrência do Lema 1 (página 9), a árvore de busca de qualquer estratégia de busca para o jogo $\mathcal{J}(P, P)$ tem altura pelo menos $\lfloor\lg n\rfloor+1$. 


\section{Capítulo 2}

\section{Ordens Aleatórias: Modelos Probabilísticos para Ordens}

Mostramos, no Capítulo 1, que o BOOP é $\mathcal{N} \mathcal{P}$-difícil. Duas abordagens comuns para procurar soluções satisfatórias para um problema $\mathcal{N} \mathcal{P}$-difícil são o recurso a algoritmos de aproximação e a análise de caso médio de algoritmos cujo desempenho no pior caso não é satisfatório. No primeiro caso a idéia é propor algoritmos que apesar de não encontrar uma solução ótima para o problema, encontram soluções cuja diferença quanto à solução ótima é controladamente limitada. No segundo caso a idéia é propor algoritmos que apesar de não encontrar uma solução ótima para todas as instâncias do problema, apresentam desempenho médio satisfatório. Mais precisamente, assumida alguma distribuição de probabilidades para o conjunto de possíveis instâncias do problema, demonstra-se que o evento de esse algoritmo não determinar uma solução satisfatória para o problema em tempo polinomial tem baixa probabilidade. Neste trabalho combinamos elementos de ambas as abordagens. Apresentamos algoritmos polinomiais e mostramos que tais algoritmos fornecem boas soluções aproximadas para instâncias típicas do problema.

Falar em "instância típica do problema" leva subentendida uma distribuição de probabilidades para o conjunto de instâncias do BOOP, ou seja, para o conjunto das ordens de um dado tamanho. Noutras palavras, para fazer uma análise de caso médio de um algoritmo para o BOOP é necessário estabelecer um modelo probabilístico para ordens: um modelo de ordens aleatórias. Existem diversos modelos de ordens aleatórias de interesse. Entretanto, ao contrário do que acontece com modelos probabilísticos para 
outros objetos combinatórios como grafos, permutações etc, no caso das ordens esses diversos modelos não são equivalentes entre si nem são redutíveis uns aos outros. Mais ainda, nenhum deles se impõe como o "mais natural" ou "o mais conveniente".

Neste trabalho concentramo-nos em dois dos modelos de ordens aleatórias que talvez se possa chamar de "os mais clássicos": o modelo grafo-aleatório e o modelo uniforme. Apresentamos estes modelos brevemente neste capítulo, enunciando alguns resultados que serão utilizados nos capítulos seguintes. O leitor familiarizado com estes modelos probabilísticos pode permitir-se uma leitura superficial do presente capítulo com o único propósito de inteirar-se da notação utilizada e dos resultados postos em destaque.

No Capítulo 6 fazemos alguns comentários adicionais a respeito de ordens aleatórias e indicamos referência bibliográfica para o assunto.

\subsection{O Modelo Grafo-Aleatório}

Seja $G$ um grafo com $V(G)=\{1, \ldots, n\}$ para algum $n>0$. Podemos definir uma ordem $P_{G}$ a partir de $G$ fazendo $P_{G}=V(G)=\{1, \ldots, n\}$ e definindo para cada aresta $u v \in G$ que $u \prec_{P_{G}} v$ quando $u<v$, isto é, definindo $\prec_{P_{G}}$ como o fecho transitivo da relação $\{(u, v):\{u, v\} \in E(G), u<v\}$.

A partir dessa construção, podemos definir um modelo de ordens aleatórias a partir de um modelo de grafos aleatórios. O modelo de ordens grafo-aleatórias ("random graph orders"), denotado $\mathcal{P}_{n, p}$, é o modelo de ordens aleatórias que se obtém a partir dessa construção quando se parte do conhecido modelo de grafos aleatórios de Erdős e Rényi, usualmente denotado por $\mathcal{G}_{n, p}$ (veja, por exemplo, Janson et al. (2000)).

Antes de prosseguir, é muito importante ressaltar que o presente trabalho ocupa-se de $\mathcal{P}_{n, p}$ somente nos casos em que p é uma constante com relação $a n$. A fim de não carregar demasiado o texto repetindo esta precisão a cada passo, tomamos a liberdade de dá-la por assente pelo restante desta parte do trabalho.

As ordens grafo-aleatórias gozam de um certo apelo intuitivo no sentido de se apresentarem como um modelo probabilístico bastante natural para ordens. Além disso, o "parentesco próximo" com o modelo $\mathcal{G}_{n, p}$ para grafos aleatórios, terreno já bastante explorado e mapeado, faz esse modelo ainda mais atraente na medida em que resultados e técnicas utilizados no estudo de $\mathcal{G}_{n, p}$ podem ser aproveitados ou adaptados para o estudo de $\mathcal{P}_{n, p}$. 
A principal dificuldade técnica no estudo de ordens aleatórias reside na ausência de independência entre os eventos da forma $i \prec j$, decorrente da transitividade. Mais precisamente, a dificuldade consiste em que a ocorrência dos eventos $i \prec j$ e $j \prec k$ numa ordem determina a ocorrência de $i \prec k$. Como veremos, essa característica das ordens aleatórias (presente em qualquer modelo que se use) leva a resultados que podem parecer um pouco surpreendentes à primeira vista. A título de ilustração no caso das ordens grafo-aleatórias, considere os seguintes resultados.

Lema 2.1. Para todo $n>0, \mathcal{G}_{n, 1 / 2}$ é um espaço uniforme de probabilidades.

Demonstração. O espaço de probabilidades $\mathcal{G}_{n, 1 / 2}$ é formado pelos grafos $G$ com $V(G)=\{1, \ldots, n\}$. Existem $2\left(\begin{array}{c}n \\ 2\end{array}\right)$ tais grafos. Para todo $k \geq 0$, a probabilidade de um grafo de $k$ arestas de $\mathcal{G}_{n, 1 / 2}$ é $2^{-k} 2^{-\left(\left(\begin{array}{c}n \\ 2\end{array}\right)-k\right)}=2^{-\left(\begin{array}{l}n \\ 2\end{array}\right)}$.

Lema 2.2. Não existe $0<p<1$ tal que $\mathcal{P}_{n, p}$, seja um espaço uniforme de probabilidades para todo $n>0$.

Demonstração. Seja $n>0$ dado. A probabilidade da ordem vazia em $\mathcal{P}_{n, p}$

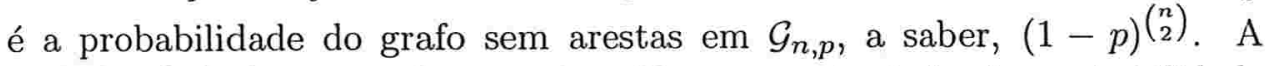
probabilidade de uma ordem total em $\mathcal{P}_{n, p}$, por outro lado, é a probabilidade da ocorrência do caminho $(1, \ldots, n)$ em $\mathcal{G}_{n, p}$, a saber, $p^{n-1}$.

Para que $\mathcal{P}_{n, p}$ seja um espaço uniforme, estes dois eventos devem ter a mesma probabilidade, o que nos leva a $(1-p)^{\left(\begin{array}{l}n \\ 2\end{array}\right)}=p^{n-1}$ para todo $n>0$ ou, equivalentemente, $p+p^{2 / n}=1$ para todo $n>0$, o que não admite uma solução $0<p<1$.

No que segue enunciamos algumas propriedades típicas de ordens grafoaleatórias. Estes resultados serão utilizados na análise dos algoritmos apresentados no Capítulo 3.

Uma maneira de estudar $\mathcal{P}_{n, p}$ é através do estudo do modelo $\mathcal{P}_{\mathbb{Z}, p}$. Como sugere a notação, $\mathcal{P}_{\mathbb{Z}, p}$ é o modelo de ordens aleatórias que se obtém a partir dos grafos aleatórios $\mathcal{G}_{\mathbb{Z}, p}$ de acordo com a mesma construção explicada no início desta seção. Os grafos aleatórios $\mathcal{G}_{\mathbb{Z}, p}$, por sua vez, são grafos aleatórios como os de $\mathcal{G}_{n, p}$, onde o conjunto de vértices é $\mathbb{Z}$ em vez de $\{1, \ldots, n\}$.

Teorema 2.3 (Alon, Bollobás, Brightwell, and Janson (1994)). As distâncias entre postos consecutivos de $\mathcal{P}_{\mathbb{Z}, p}$ são variáveis aleatórias independentes $e$ identicamente distribuídas. 
Teorema 2.4 (Kim and Pittel (2000)). Seja L uma variável aleatória com a mesma distribuição das distâncias entre postos consecutivos de $P_{\mathbb{Z}, p}$. Existe uma constante $c_{1}=c_{1}(p)>0$ tal que, para todo $l>0$,

$$
\mathbb{P}(L>l)<\exp \left(-c_{1} l\right) \text {. }
$$

Corolário 2.5. A ordem típica de $\mathcal{P}_{n, p}, 0<p<1$ não tem segmentos com mais do que $c_{2} \log n$ elementos para todo $c_{2}=c_{2}(p)>\left(1 / c_{1}(p)\right)$, onde $c_{1}(p)$ é a mesma constante do enunciado do Teorema 2.4 .

Demonstração. Seja $P$ uma ordem grafo-aleatória e seja $c_{2}$ como no enunciado do Corolário 2.5. Seja ainda $k=|\pi(P)|$. Para cada $1 \leq i \leq k$ temos que $\left|S_{i}(P)\right|$ é uma variável aleatória, sendo $\left|S_{0}(P)\right|=\pi_{1}(P)-1$, $\left|S_{k}(P)\right|=n-\pi_{k}(P)$ e $\left|S_{i}(P)\right|=\pi_{i+1}(P)-\pi_{i}(P)-1$ para todo $1<i<k$.

Segue do Teorema 2.4 que essas variáveis aleatórias também satisfazem a desigualdade (2.1) e, portanto, para todo $1 \leq i \leq k$ temos

$$
\mathbb{P}\left(\left|S_{i}(P)\right|>c_{2} \log n\right)<\exp \left(-c_{1} c_{2} \log n\right)=n^{-c_{1} c_{2}},
$$

e assim,

$\mathbb{P}$ ( $P$ tem algum segmento com mais de $c_{2} \log n$ elementos $)$

$$
\begin{aligned}
=\mathbb{P}\left(\bigvee_{i=0}^{k}\left\{\left|S_{i}(P)\right|>c_{2} \log n\right\}\right) & \leq \sum_{i=0}^{k} \mathbb{P}\left(\left|S_{i}(P)\right|>c_{2} \log n\right) \\
<(k+1) n^{-c_{1} c_{2}} & \leq(n+1) n^{-c_{1} c_{2}}=n^{1-c_{1} c_{2}}+n^{-c_{1} c_{2}},
\end{aligned}
$$

e portanto, como $c_{2}>\left(1 / c_{1}\right)$,

$\lim _{n \rightarrow \infty} \mathbb{P}$ ( $P$ tem algum segmento com mais de $c_{2} \log n$ elementos $)$

$$
=\lim _{n \rightarrow \infty} n^{1-c_{1} c_{2}}+n^{-c_{1} c_{2}}=0 .
$$

Teorema 2.6 (Bollobás and Brightwell (1995)). Dados $0<p<1$ e $n>0$, seja $K_{n}$ a solução de $n(1-p)^{\left(\begin{array}{c}K_{n} \\ 2\end{array}\right)}=1$, isto é,

$$
K_{n}=\sqrt{\frac{2 \log n}{\log (1 /(1-p))}+\frac{1}{4}}+\frac{1}{2} .
$$

Para todo $\delta>0$, a largura da ordem grafo-aleatória típica está compreendida no intervalo $\left[\left\lfloor K_{n}-\delta\right\rfloor,\left[K_{n}+\delta\right\rceil\right]$. 
Corolário 2.7. A largura da ordem grafo-aleatória típica não é maior que

$$
\sqrt{\frac{2 \log n}{\log (1 /(1-p))}}+1
$$

\subsection{O Modelo Uniforme}

Para cada $n>0$ seja $\mathcal{P}(n)$ o conjunto de todas as ordens sobre $\{1, \ldots, n\}$. O modelo uniforme de ordens aleatórias é a família de espaços de probabilidade $\{\mathcal{U}(\mathcal{P}(n)): n>0\}$. Para cada $n>0$ denotamos $\mathcal{U}(\mathcal{P}(n))$ por $\mathcal{U}_{n}$.

Se dissemos que as ordens grafo-aleatórias gozam de um certo "apelo intuitivo" no sentido de que parecem um modelo probabilístico bastante natural para ordens, por que não dizer o mesmo a respeito do modelo uniforme? Afinal de contas o espaço de probabilidades uniformemente gerado por um conjunto de objetos parece sempre o candidato ao espaço de probabilidades que se impõe como o "mais natural". Entretanto, como veremos a seguir, a ordem típica no modelo uniforme de ordens aleatórias é tão "pouco natural", no sentido de que contraria a idéia ingênua de "como deveria ser", que faz hesitar em conferir ao modelo uniforme essa preferência.

Para descrever a ordem típica de $\mathcal{U}_{n}$ vamos definir uma família de ordens sobre $\{1, \ldots, n\}$ que chamaremos ordens tripartidas. A seguir, enunciamos um resultado fundamental de Kleitman and Rothschild (1975) que prova que a ordem típica de $\mathcal{U}_{n}$ é uma ordem tripartida com certas características especiais.

Dado $n>0$, seja $\left\{X_{1}, X_{2}, X_{3}\right\}$ uma partição de $\{1, \ldots, n\}$. Vamos denotar por $\mathcal{A}\left(X_{1}, X_{2}, X_{3}\right)$ o conjunto das ordens $P$ sobre $\{1, \ldots, n\}$ satisfazendo

1. todo $x_{3} \in X_{3}$ é menor do que todo $x_{1} \in X_{1}$;

2. se $x_{i} \in X_{i}, x_{j} \in X_{j}$, e $x_{i} \prec_{P} x_{j}$, então $i>j$.

Dizemos que uma ordem $P$ sobre $\{1, \ldots, n\}$ é uma ordem tripartida se existe uma partição $\left\{X_{1}, X_{2}, X_{3}\right\}$ de $\{1, \ldots, n\}$ tal que $P \in \mathcal{A}\left(X_{1}, X_{2}, X_{3}\right)$. Denotaremos por $\mathcal{T}_{n}$ a família de espaços uniformes gerados pelas ordens tripartidas sobre $\{1, \ldots, n\}$, isto é,

$$
\mathcal{T}_{n}=\left\{\mathcal{U}\left(\mathcal{A}\left(X_{1}, X_{2}, X_{3}\right)\right):\left\{X_{1}, X_{2}, X_{3}\right\} \text { é partição de }\{1, \ldots, n\}\right\} \text {. }
$$

Em busca de uma caracterização da ordem típica do modelo uniforme, Kleitman and Rothschild (1975) provaram o seguinte resultado fundamental. 
Teorema 2.8 (Kleitman and Rothschild (1975)). A ordem típica do modelo uniforme é uma ordem tripartida que pertence a $\mathcal{A}\left(X_{1}, X_{2}, X_{3}\right)$ para alguma partição $\left\{X_{1}, X_{2}, X_{3}\right\}$ de $\{1, \ldots, n\}$ satisfazendo

$$
\begin{aligned}
& || X_{2}\left|-\frac{n}{2}\right|<\omega(n), \\
& || X_{3}\left|-\frac{n}{4}\right|<\omega(n) \sqrt{n},
\end{aligned}
$$

para qualquer $\omega: \mathbb{N} \rightarrow \mathbb{N}$ tal que $\lim _{n \rightarrow \infty} \omega(n)=\infty$.

Com certa dose de liberdade de expressão, podemos dizer que a ordem típica no modelo uniforme é uma ordem tripartida onde a "classe do meio" comporta aproximadamente a metade dos elementos.

$\mathrm{O}$ estudo de certas propriedades típicas em $\mathcal{U}_{n}$ fica bastante facilitado se feito indiretamente, estudando modelos probabilísticos correlatos. No que segue, vamos mostrar que toda propriedade típica de $\mathcal{T}_{n}$ é também uma propriedade típica de $\mathcal{U}_{n}$. A seguir, mostramos que $\mathcal{T}_{n}$ é, por sua vez, equivalente a um modelo probabilístico para grafos bipartidos cujo estudo é bastante mais fácil do ponto de vista técnico. Este resultado será utilizado na análise dos algoritmos apresentados no Capítulo 4. Pelo resto desta seção, usamos $\omega: \mathbb{N} \rightarrow \mathbb{N}$ para denotar uma função satisfazendo $\lim _{n \rightarrow \infty} \omega(n)=\infty$.

Seja $\Pi_{n}(\omega)$ o conjunto das triplas $\left(X_{1}, X_{2}, X_{3}\right)$ tais que $\left\{X_{1}, X_{2}, X_{3}\right\}$ é uma partição de $\{1, \ldots, n\}$ satisfazendo (2.3) e (2.4).

Fazendo

$$
\mathcal{A}_{\mathrm{KR}}(n, \omega)=\bigcup_{\left(X_{1}, X_{2}, X_{3}\right) \in \Pi_{n}(\omega)} \mathcal{A}\left(X_{1}, X_{2}, X_{3}\right),
$$

temos pelo Teorema 2.8 que

$$
\lim _{n \rightarrow \infty} \frac{\left|\mathcal{A}_{\mathrm{KR}}(n, \omega)\right|}{|\mathcal{P}(n)|}=1
$$

O seguinte argumento padrão mostra que podemos estudar propriedades típicas de $\mathcal{U}_{n}$ restringindo nossa atenção a $\mathcal{A}_{\mathrm{KR}}(n, \omega)$.

Lema 2.9. Seja $\mathcal{E} \subseteq \mathcal{P}(n)$ um evento tal que a probabilidade de $U_{n} \in$ $\mathcal{E}$, condicionada a $U_{n} \in \mathcal{A}\left(X_{1}, X_{2}, X_{3}\right)$, tende a 1 à medida que $n \rightarrow \infty$ uniformemente em $\left(X_{1}, X_{2}, X_{3}\right) \in \Pi_{n}(\omega)$. Então, quase certamente $U_{n} \in \mathcal{E}$. 
Demonstração. Usamos a hipótese sobre $\mathcal{E}$ e o Teorema 2.8 para observar que, em $\mathcal{U}_{n}$, temos

$$
\begin{aligned}
\mathbb{P}(\overline{\mathcal{E}}) & =\mathbb{P}\left(\overline{\mathcal{E}} \cap \mathcal{A}_{\mathrm{KR}}(n, \omega)\right)+\mathbb{P}\left(\overline{\mathcal{E}} \cap \overline{\mathcal{A}_{\mathrm{KR}}(n, \omega)}\right) \\
\leq & \sum_{\Pi_{n}(\omega)} \mathbb{P}\left(\overline{\mathcal{E}} \mid \mathcal{A}\left(X_{1}, X_{2}, X_{3}\right)\right) \mathbb{P}\left(\mathcal{A}\left(X_{1}, X_{2}, X_{3}\right)\right)+\mathbb{P}\left(\overline{\mathcal{A}_{\mathrm{KR}}(n, \omega)}\right) \\
& =o(1) \sum_{\Pi_{n}(\omega)} \mathbb{P}\left(\mathcal{A}\left(X_{1}, X_{2}, X_{3}\right)\right)+o(1)=o(1),
\end{aligned}
$$

onde os somatórios acima são sobre todo $\left(X_{1}, X_{2}, X_{3}\right) \in \Pi_{n}(\omega)$ (veja (2.5)).

Sejam $X$ e $Y$ dois conjuntos disjuntos e seja $\mathcal{A}(X, Y)$ o conjunto das ordens $P$ sobre $X \cup Y$ tais que $y \prec_{P} x$ somente se $y \in Y$ e $x \in X$.

Dada uma partição $\left\{X_{1}, X_{2}, X_{3}\right\}$ de $\{1, \ldots, n\}$, temos que $\mathcal{A}\left(X_{1}, X_{2}\right) \times \mathcal{A}\left(X_{2}, X_{3}\right)$ e $\mathcal{A}\left(X_{1}, X_{2}, X_{3}\right)$ são equivalentes, no sentido em que existe uma bijeção natural que associa a cada ordem de $\mathcal{A}\left(X_{1}, X_{2}, X_{3}\right)$ um par de ordens de $\mathcal{A}\left(X_{1}, X_{2}\right) \times \mathcal{A}\left(X_{2}, X_{3}\right)$. Isto porque a definição de $\mathcal{A}\left(X_{1}, X_{2}, X_{3}\right)$ implica que cada ordem em $P \in \mathcal{A}\left(X_{1}, X_{2}, X_{3}\right)$ está completamente determinada pelo conjunto de elementos comparáveis entre as "partições extremas" e a "partição do meio", isto é, pelo conjunto $\prec_{P} \cap\left(X_{1} \times X_{2} \cup X_{2} \times X_{3}\right)$.

Temos então que esses conjuntos têm o mesmo número de elementos, a saber $2^{\left|X_{1}\right|\left|X_{2}\right|+\left|X_{2}\right|\left|X_{3}\right|}$, e, portanto, os espaços uniformes gerados por cada um deles são probabilisticamente equivalentes.

Por outro lado, é fácil ver que no espaço $\mathcal{U}\left(\mathcal{A}\left(X_{1}, X_{2}\right) \times \mathcal{A}\left(X_{2}, X_{3}\right)\right)$ temos, com independência entre os eventos,

$$
\mathbb{P}\left(y \prec_{P} x\right)= \begin{cases}\frac{1}{2}, & \text { se } y \in X_{i+1} \text { e } x \in X_{i}, 1 \leq i<3 ; \\ 0, & \text { caso contrário, }\end{cases}
$$

observação que nos será útil na Seção 4. 


\section{Capítulo 3}

\section{Busca Ótima em Ordens Grafo-Aleatórias}

Neste capítulo apresentamos e analisamos um algoritmo polinomial para o BOOP e mostramos que este algoritmo fornece uma $(1+o(1))$-aproximação de uma solução ótima para a instância grafo-aleatória típica.

Seja $P$ uma ordem. Definimos a $h(P)$-ésima camada de $P$ como sendo o conjunto dos elementos maximais de $P$. Para cada $1 \leq k<h(P)$, definimos a $k$-ésima camada de $P$, denotada $C_{k}(P)$, como o conjunto dos elementos de $P$ cobertos pelos elementos da $(k+1)$-ésima camada de $P$.

Para todo $1 \leq k \leq h(P)$, definimos as camadas abaixo de $C_{k}$ por

$$
C_{k \downarrow}(P)=\left|\bigcup_{i=1}^{k-1} C_{i}(P)\right|,
$$

e as camadas acima de $C_{k}$ por

$$
C_{k \uparrow}(P)=\left|\bigcup_{i=k+1}^{h(P)} C_{i}(P)\right|,
$$

e por fim, definimos uma camada mediana de $P$ como uma camada $C_{m}$ de $P$ satisfazendo $\max \left\{C_{m \downarrow}(P), C_{m \uparrow}(P)\right\} \leq|P| / 2$.

A estratégia de busca $\mathcal{B}$ para o jogo $\mathcal{J}$ que apresentamos a seguir faz uso do fato de que o conjunto das camadas de uma ordem particiona seus elementos. Numa descrição informal, essa estratégia procura um elemento $u$ numa ordem $P$, fazendo perguntas a cada elemento de uma camada mediana 
de $P$. Conforme as respostas recebidas, a estratégia descarta as camadas acima ou abaixo da camada mediana, e recomeça a busca a partir de uma camada mediana da ordem induzida pelos elementos restantes. Note que se $P$ é uma ordem total, a Estratégia $\mathcal{B}$ nada mais é que a conhecida Estratégia de Busca Binária, motivo pelo qual chamamo-la de Busca Binária Parcial.

\section{Estratégia $\mathcal{B}(P, S)$}

Se $S=\emptyset$, o jogo termina $(u \notin P[S])$

$m \leftarrow$ índice de uma camada mediana de $P[S]$

pergunte $c$ para cada $c \in C_{m}(P[S])$

se a resposta for $=$, o jogo termina $\left(u=c \in C_{m}(P[S]) \subseteq S\right)$

se a resposta for $\prec$

aplique a Estratégia $\mathcal{B}$ ao jogo $\mathcal{J}\left(P, I^{-}(c)\right)$; o jogo termina com o mesmo resultado deste jogo

aplique a Estratégia $\mathcal{B}$ ao jogo $\mathcal{J}\left(P, C_{m \uparrow}\right)$; o jogo termina com o mesmo resultado desse jogo

Teorema 3.1. É possível computar em tempo polinomial uma árvore de decisão de altura máxima $w(P)(\lfloor\lg h(P)\rfloor+1)$ para uma ordem $P$, a partir de seu diagrama de Hasse.

Demonstração. Seja $P$ uma ordem e considere um algoritmo que computa a árvore de decisão correspondente à estratégia $\mathcal{B}$ para o jogo $\mathcal{J}(P, P)$ a partir do diagrama de Hasse de $P$.

Esquematicamente, tal algoritmo poderia ser estruturado segundo os seguintes passos:

1. decomponha $P$ em camadas computando o número de elementos de cada camada, e determine o índice $m$ de uma camada mediana de $P$.

2. compute a árvore de decisão de $P$ a partir de $C_{m}(P)$.

O passo 1 pode ser executado em tempo polinomial através de uma busca em largura sobre o grafo $\mathcal{H}(P)$.

$\mathrm{O}$ passo 2 pode ser esquematicamente estruturado da seguinte maneira. 
Seja $C_{m}=\left\{c_{1}, \ldots, c_{k}\right\}$. Para cada $1 \leq i \leq k$, denote por $T_{i \downarrow}$ a árvore de decisão de $P\left[I^{-}\left(c_{i}\right)\right]$ e por $T_{m \uparrow}$ a árvore de decisão de $P\left[C_{m \uparrow}\right]$.

Compute recursivamente a árvore de decisão $T_{k}=\left(c_{k}, T_{m \uparrow}, T_{k \downarrow}\right)$. A seguir, cada $1 \leq i<k$, em ordem decrescente, compute $T_{i}$ como sendo a árvore de decisão dada por $T_{i}=\left(c_{i}, T_{i \downarrow}, T_{i+1}\right)$.

Cada recursão deste algoritmo acrescenta no máximo $w(P)$ níveis à árvore de decisão, um para cada elemento da camada mediana. Como o maior nível de recursão aninhada atingido pelo algoritmo é $\lfloor\lg h(P)\rfloor+1$, podemos concluir que a altura da árvore computada é no máximo

$$
w(P)(\lfloor\lg h(P)\rfloor+1) .
$$

Finalmente, como a construção da árvore de decisão $T_{i}$ pode ser feita em tempo polinomial para cada $1 \leq i \leq k$, temos que o algoritmo como um todo é polinomial.

A estratégia de busca $\mathcal{A}$ para o jogo $\mathcal{J}$ que apresentamos a seguir faz uso do fato de que o último lance do jogo na Estratégia $\mathcal{B}$ fornece uma indicação de "onde deveria estar" o elemento procurado. Numa descrição informal, a Estratégia $\mathcal{A}$ inicialmente aplica a Estratégia $\mathcal{B}$ à ordem total induzida por $P$ sobre o conjunto de seus postos. Se o elemento procurado não é um dos postos de $P$, o último lance desse jogo é usado como indicação do único segmento de $P$ onde pode estar o elemento procurado. A Estratégia $\mathcal{B}$ é então novamente aplicada a esse segmento de $P$.

Estratégia $\mathcal{A}(P, S)$

$$
\begin{aligned}
& S^{\prime} \leftarrow S \\
& \text { se } \pi(P[S]) \neq \emptyset
\end{aligned}
$$

aplique a estratégia $\mathcal{B}$ ao jogo $\mathcal{J}(P, \pi(P[S]))$ e seja $\left(\pi_{j}(P[S]), a\right)$ o último lance deste jogo

$$
\begin{aligned}
& \text { se } a \text { é }=\text {, o jogo termina }\left(u=\pi_{j}(P[S]) \in P[S]\right) \\
& \text { caso contrário } S^{\prime} \leftarrow S_{j-1}(P[S])
\end{aligned}
$$

aplique a estratégia $\mathcal{B}$ ao jogo $\mathcal{J}\left(P, S^{\prime}\right)$

Teorema 3.2. É possível computar em tempo polinomial uma árvore de decisão para ordem $P$ cuja altura é no máximo

$$
\lfloor\lg |\pi(P)|\rfloor+w(P)\left\lfloor\lg \max \left\{\left|S_{i}(P)\right|: 0 \leq i \leq|\pi(P)|\right\}\right\rfloor+2,
$$


a partir do seu diagrama de Hasse.

Demonstração. Seja $P$ uma ordem e considere um algoritmo que produz a árvore de decisão correspondente à estratégia $\mathcal{A}$ para o jogo $\mathcal{J}(P, P)$ a partir do diagrama de Hasse de $P$.

A árvore de decisão $T$ produzida por esse algoritmo tem os postos de $P$ nos nós de seus primeiros $\lfloor\lg |\pi(P)|\rfloor+1$ níveis e as árvores de decisão dos segmentos de $P$ como filhos desses nós. Assim

$$
h(T) \leq\lfloor\lg |\pi(P)|\rfloor+1+\max \left\{h\left(T_{i}\right): 0 \leq i \leq|\pi(P)|\right\},
$$

onde $T_{i}$ denota a árvore de decisão da ordem $P\left[S_{i}(P)\right]$ para cada $0 \leq i \leq|\pi(P)|$.

De acordo com o Teorema 3.1, para cada $0 \leq i \leq|\pi(P)|$ temos

$$
w\left(P\left[S_{i}(P)\right]\right)\left(\left\lfloor\lg h\left(P\left[S_{i}(P)\right]\right)\right\rfloor+1\right) \leq w(P)\left\lfloor\lg \left|S_{i}(P)\right|\right\rfloor+1,
$$

e portanto,

$\max \left\{h\left(T_{i}\right): 0 \leq i \leq|\pi(P)|\right\} \leq w(P) \max \left\{\left\lfloor\lg \left|S_{i}(P)\right|\right\rfloor: 0 \leq i \leq|\pi(P)|\right\}+1$.

Voltando a (3.1) ficamos com

$$
h(T) \leq\lfloor\lg |\pi(P)|\rfloor+w(P) \max \left\{\left\lfloor\lg \left|S_{i}(P)\right|\right\rfloor: 0 \leq i \leq|\pi(P)|\right\}+2 .
$$

Teorema 3.3. A árvore de busca correspondente à estratégia $\mathcal{A}$ tem altura no máximo $\lg n+O(\sqrt{\log n} \log \log n)$ para ordem grafo-aleatória típica de $n$ elementos.

Demonstração. Seja $P$ uma ordem grafo-aleatória típica de $n$ elementos e seja $T$ a árvore de decisão de $P$ correspondente à Estratégia $\mathcal{A}$. Do Teorema 3.2 temos

$$
h(T) \leq\lfloor\lg |\pi(P)|\rfloor+w(P)\left\lfloor\lg \max \left\{\left|S_{i}(P)\right|: 0 \leq i \leq|\pi(P)|\right\}\right\rfloor+2 .
$$

Do Corolário 2.7 temos

$$
w(P) \leq \sqrt{\frac{2 \log n}{\log (1 /(1-p))}}+1 .
$$


Seja $c_{2}=c_{2}(p)>\left(1 / c_{1}(p)\right)$, onde $c_{1}(p)$ é a mesma constante do enunciado do Teorema 2.4. Do Corolário 2.5 temos

$$
\max \left\{\left|S_{i}(P)\right|: 0 \leq i \leq|\pi(P)|\right\} \leq c_{2} \log n .
$$

Voltando a (3.2) ficamos com

$$
\begin{array}{r}
h(T) \leq\lfloor\lg |\pi(P)|\rfloor+\left(\sqrt{\frac{2 \log n}{\log (1 /(1-p))}}+1\right) \lg c_{2} \log n+2 \\
\leq\lfloor\lg n\rfloor+\left(\sqrt{\frac{2 \log n}{\log (1 /(1-p))}}+1\right) \lg c_{2} \log n+2 \\
=\lg n+O(\sqrt{\log n} \log \log n) .
\end{array}
$$

Corolário 3.4. A árvore de busca correspondente à Estratégia $\mathcal{A}$ é uma $(1+o(1))$ aproximação de uma solução ótima para instâncias grafo-aleatórias típicas. 


\section{Capítulo 4}

\section{Busca Ótima em Ordens Uniformes}

Neste capítulo estudamos o problema de busca numa ordem típica do modelo uniforme. Iniciamos com o seguinte resultado negativo que segue diretamente do Teorema 2.8 (página 34).

Corolário 4.1. Qualquer árvore de busca de uma ordem típica do modelo uniforme tem altura pelo menos $(1+o(1)) n / 4$.

Demonstração. Considere a seguinte estratégia de evasão para o jogo $\mathcal{J}(P, S)$.

\section{Estratégia $\mathcal{E}(P, S)$}

$M \leftarrow$ o conjunto de elementos maximais de $P[S]$

$X \leftarrow \emptyset$

a cada pergunta $p$

se $p \notin M$ responda $\npreceq$,

caso contrário,

$$
X \leftarrow X \cup\{p\}
$$

se $X=M$, responda $=$,

caso contrário, responda $\npreceq$

É imediato da formulação da Estratégia $\mathcal{E}$ que a duração de qualquer realização do jogo $\mathcal{J}(P, S)$ em que ela seja adotada é pelo menos $|M(P[S])|$, 
onde $M(P[S])$ denota o conjunto de elementos maximais de $P[S]$. De acordo com o Teorema 2.8 , a ordem típica do modelo uniforme tem $(1+o(1)) n / 4$ elementos maximais.

A fim de tornar o problema mais interessante no modelo uniforme, passamos a considerar uma variante do BOOP, que descrevemos por meio do jogo de esconder associado.

Jogo: busca em ordens parciais $\left(\mathcal{J}^{\prime}(P, S)\right)$

Início: ambos jogadores recebem uma ordem $P$ e um conjunto $S \subseteq P$; a ocultadora escolhe um elemento $u \in P$

Lance: cada pergunta é um elemento $s \in S$; a resposta é $=, \prec, \succ$ ou $\nsim$, conforme $s=u, s \prec_{P} u, s \succ_{P} u$ ou $s \nsim u$ respectivamente

Fim: o procurador encontra-se em condições de declarar se $u \in S$

Observe que uma árvore de busca para o jogo $\mathcal{J}^{\prime}$ é uma árvore ternária e temos a seguinte decorrência imediata do Lema 1 (página 9).

Corolário 4.2. Qualquer árvore de busca para o jogo $\mathcal{J}^{\prime}$ sobre uma ordem de $n$ elementos tem altura pelo menos $\left\lfloor\log _{3} n\right\rfloor+1$.

Neste capítulo vamos provar que é possível construir em tempo polinomial uma árvore de busca de altura $O(\log n)$ para uma ordem típica do modelo uniforme com $n$ elementos.

\subsection{Busca em Ordens Tripartidas}

Seja $P$ uma ordem e seja $S \subseteq P$. Começamos por apresentar a estratégia de busca $\overline{\mathcal{C}}$ para o jogo $\mathcal{J}^{\prime}$. A estratégia $\overline{\mathcal{C}}$ recebe como informação, além da ordem $P$ e do conjunto $S$, dois subconjuntos disjuntos $Q$ e $R$ de $S$. O objetivo da estratégia $\overline{\mathcal{C}}$ é determinar se o elemento procurado pertence a $Q \cup R$. Isso é feito da seguinte maneira. O procurador faz uma pergunta para cada elemento de $Q$ e usa cada resposta para eliminar "candidatos potenciais" de $R$. Se após esgotar o conjunto $Q$ o elemento procurado não foi achado, o procurador faz uma pergunta para cada um dos elementos remanescentes em $R$. 


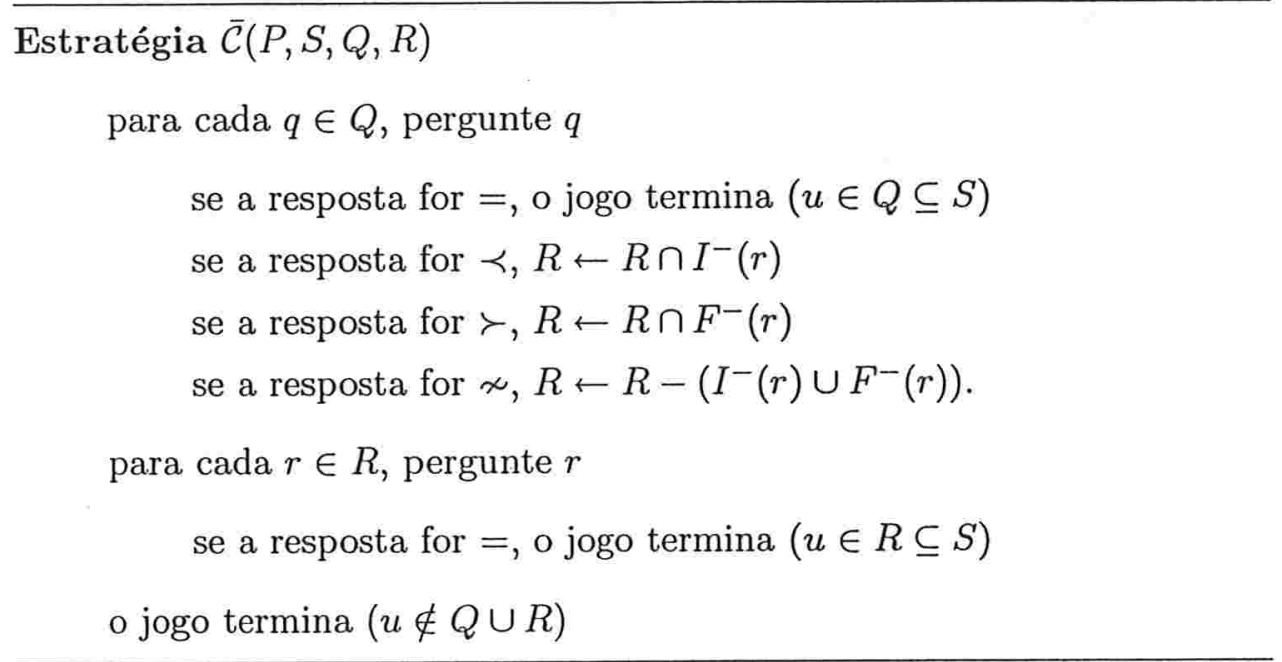

Vamos mostrar que para uma ordem típica de $\mathcal{U}_{n}$ é possível escolher $Q$ e $R$ de maneira a garantir que 2 aplicações consecutivas da estratégia $\overline{\mathcal{C}}$ resultam num jogo de duração $O(\log n)$.

Lema 4.3. Seja $P \in \mathcal{A}\left(X_{1}, X_{2}, X_{3}\right) \subseteq \mathcal{A}_{\mathrm{KR}}(n, \omega)$ e seja $Q \subseteq X_{1}$ fixo. Se $N$ é o número de elementos $x \in X_{2}$ com uma dada relação de comparabilidade/incomparabilidade com todo $q \in Q$, então sua esperança é

$$
\mathbb{E}[N]=\left|X_{2}\right| 2^{-|Q|} .
$$

Lema 4.4. Seja $P \in \mathcal{A}\left(X_{1}, X_{2}, X_{3}\right) \subseteq \mathcal{A}_{\mathrm{KR}}(n, \omega)$ uma ordem típica do modelo uniforme e seja $Q$ um subconjunto de $X_{2}$ de cardinalidade $q(n)=\lceil 2 \lg n+\omega(n)\rceil$. A estratégia $\overline{\mathcal{C}}\left(P, S, Q, X_{1} \cup X_{3}\right)$ quase certamente faz no máximo $q(n)+1$ perguntas no jogo $\mathcal{J}^{\prime}(P, P)$. Além disso, se $Q$ é um subconjunto de $X_{1} \cup X_{3}$ de cardinalidade $q(n)$, então a estratégia $\overline{\mathcal{C}}\left(P, S, Q, X_{2}\right)$ quase certamente faz no máximo $q(n)+1$ perguntas no jogo $\mathcal{J}^{\prime}(P, P)$.

Demonstração. Denotando por $r(Q, R)$ o conjunto dos elementos restantes em $R$ na execução da estratégia $\overline{\mathcal{C}}(P, S, Q, R)$, após todas as perguntas em $Q$ terem sido feitas, temos que o número de perguntas feitas pela estratégia $\overline{\mathcal{C}}(P, S, Q, R)$ é no máximo $|Q|+|r(Q, R)|$.

Seja $Z$ o número de pares $(x, y) \in R \times R$ tais que , $x \neq y$ e $\{x, y\} \subset r(Q, R)$. Vê-se facilmente (lembrando (4.1)) que a esperança de $Z$ é no máximo $|R|^{2} 2^{-|Q|} \leq n^{2} 2^{-q(n)}=o(1)$. Portanto, $Z=0 \mathrm{com}$ probabilidade $1-o(1)$, e daí quase certamente, $|r(Q, R)| \leq 1$. 
Com isso podemos formular a estratégia de busca $\overline{\mathcal{D}}_{\omega}$ para o jogo $J^{\prime}$ que procede da seguinte maneira. Inicialmente o procurador verifica se $P[S] \in \mathcal{A}\left(X_{1}, X_{2}, X_{3}\right) \subseteq \mathcal{A}_{\mathrm{KR}}(|S|, \omega)$ para alguma partição $\left\{X_{1}, X_{2}, X_{3}\right\}$ de $S$. Em caso negativo, ele aplica a estratégia $\mathcal{B}^{\prime}$ ao jogo (chamamos de estratégia $\mathcal{B}^{\prime}$ à trivial adaptação da estratégia de busca $\mathcal{B}$, do Capítulo 3 , para o jogo $\left.\mathcal{J}^{\prime}\right)$. Se, pelo contrário, $P[S] \in \mathcal{A}\left(X_{1}, X_{2}, X_{3}\right) \subseteq \mathcal{A}_{\mathrm{KR}}(|S|, \omega)$ para alguma partição $\left\{X_{1}, X_{2}, X_{3}\right\}$ de $S$, então o procurador seleciona um subconjunto $Q$ de tamanho $\lceil 2 \lg n+\omega(n)\rceil$ de $X_{2}$ e aplica a estratégia $\overline{\mathcal{C}}\left(P, S, Q, X_{1} \cup X_{3}\right)$. Se o elemento procurado estiver em $X_{1} \cup X_{3}$, o jogo termina. Caso contrário, ele seleciona um subconjunto $Q^{\prime}$ de $X_{1} \cup X_{3}$, também de tamanho $\lceil 2 \lg n+\omega(n)\rceil$ e aplica a estratégia auxiliar $\overline{\mathcal{C}}\left(P, S, Q^{\prime}, X_{2}\right)$. Isso termina o jogo, uma vez que decide se o elemento procurado pertence ou não a $X_{2}$.

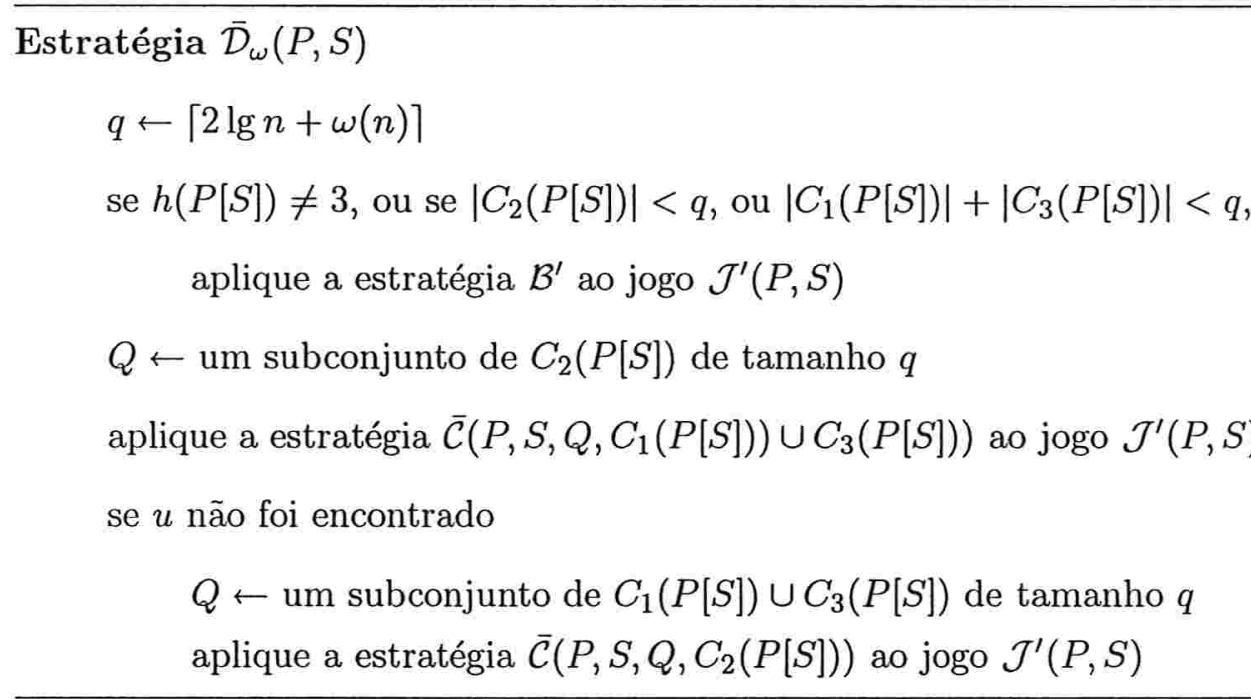

Teorema 4.5. A árvore de busca correspondente à estratégia $\overline{\mathcal{D}}_{\omega}$ tem altura no máximo $6.34 \log _{3} n$ para ordem típica de $n$ elementos do modelo uniforme.

Demonstração. Seja $P$ uma ordem típica de $n$ elementos do modelo uniforme, com $P \in \mathcal{A}_{\mathrm{KR}}(n, \omega)$. Pelo Lema 4.4, o número de perguntas feitas pela estratégia $\overline{\mathcal{D}}_{\omega}$ é no máximo

$$
2(\lceil 2 \lg n+\omega(n)\rceil)=(4+o(1)) \lg n=(6.33 \ldots+o(1)) \log _{3} n .
$$




\section{Capítulo 5}

\section{Um Problema Correlato}

Um problema correlato ao BOOP é o estudado por Linial and Saks (1985a), que designaremos aqui por Busca com Informação Parcial (BOIP) e sobre o qual fazemos algumas breves considerações neste capítulo.

Formulado como um jogo de esconder, no problema de Busca com Informação Parcial são dados aos jogadores, como no BOOP, uma ordem $P$ e um conjunto $S \subseteq P$. Como no BOOP, o objetivo do procurador é determinar se um dado elemento $x$ de $P$ pertence ou não a $S$. Diferentemente do BOOP, entretanto, aqui a ocultadora escolhe uma ordem total $T \supseteq P \mathrm{e}$ cada lance do jogo é uma pergunta relativa à ordem $T$ e não à ordem $P[S]$. Conseqüentemente a cada lance $(p, a)$ em que $a=\npreceq$, o procurador ganha a informação de que não só $p \npreceq u$ mas na verdade $u \prec p$.

A motivação original do BOIP é a seguinte. Seja uma matriz $M$ de dimensões $m \times n$ sobre $\mathbb{R}$ cujas entradas estão ordenadas segundo as linhas e segundo as colunas de forma crescente. O problema é o de, dado um valor $u$, determinar se existem índices $i$ e $j$ de $M$ tais que $M_{i}^{j}=u$. Nesse caso temos a ordem usual sobre $\mathbb{R}$ no papel da ordem $T$ e a ordem $P$ dada é o produto de uma cadeia de comprimento $m$ por uma cadeia de tamanho $n$.

Para ver por que o BOIP é de fato um problema diferente do BOOP, considere quanta informação o procurador ganha num lance do tipo $(p, \prec)$. No BOOP um tal lance é suficiente para confinar o resto da busca a $S \cap I^{-}(s)$; no BOIP, entretanto, como a pergunta é feita com respeito à ordem total $T$, essa resposta deixa todos os elementos de $S-\{x \in S: s \prec x\}$ como candidatos a serem ainda considerados pelo procurador.

Neste capítulo vamos apresentar esquematicamente um argumento que leva a concluir que, apesar de tratar-se de diferentes problemas, as es- 
tratégias e resultados relativos ao BOOP no modelo grafo-aleatório podem ser adaptados ao BOIP.

Inicialmente registramos o fato de que, como conseqüência do resultados em Linial and Saks (1985b) e Linial and Saks (1985a), a altura $H$ da árvore ótima para o problema satisfaz

$$
\lg \iota(P) \leq H \leq \kappa \lg \iota(P),
$$

onde $\iota(P)$ é o número of ideais de $P$ e $\kappa=(2-\lg (1+\lg 5))^{-1}=3.73 \ldots$.

Como um ideal de uma ordem está univocamente determinado pela anticadeia de seus elementos maximais, temos que o número de ideais num segmento $R$ de $P$ está limitado pelo número de anti-cadeias que contém, e daí

$$
\iota(P(R)) \leq \sum_{i=1}^{w(P)}\left(\begin{array}{c}
|R| \\
i
\end{array}\right) \leq|R|^{w(P)} .
$$

Combinando as cotas de (5.1) e o Teorema 2.6, isto permite-nos deduzir a existência de uma árvore para $R$ de altura no máximo $\kappa \lg \iota(P(R))=O(w(P) \lg |R|)$.

Daí, para qualquer ordem parcial, existe uma árvore de altura $H \leq \lg n+O(w(P) \lg r)$, onde $r$ é a máxima cardinalidade dos segmentos de $P$. Novamente, pelo Corolário 2.5 e pelo Teorema 2.6 , temos para a ordem grafo-aleatória típica de $n$ elementos que

$$
H \leq \lg n+O\left((\log n)^{1 / 2} \log \log n\right) .
$$




\section{Capítulo 6}

\section{Comentários Finais}

A redução do Capítulo 1 assemelha-se à empregada por Hyafil and Rivest (1976) para provar que o problema da árvore de decisão ótima é $\mathcal{N} \mathcal{P}$-completo. De fato, BOOP pode ser visto como uma restrição deste problema. É interessante também observar que a prova dada pode ser facilmente modificada de forma a contemplar o caso em que a ordem dada tem um elemento máximo.

O caso ainda mais restrito do BOOP no qual a ordem dada, além de ter um elemento máximo, apresenta um diagrama de Hasse que é uma árvore (chamado caso enraizado) pode ser resolvido em tempo polinomial, como provado por Ben-Asher et al. (1999). Neste trabalho os autores dão um algoritmo de tempo $O\left(n^{4}(\log n)^{3}\right)$ para computação de uma árvore de busca ótima, para uma ordem de $n$ elementos. Este algoritmo, entretanto, não permite uma estimativa fácil da altura da árvore computada, exceto em alguns poucos casos; por exemplo, quando o diagrama de Hasse da ordem dada é uma árvore binária completa, caso em que a árvore de busca construída tem altura $\lg n+\lg ^{*} n+\Theta(1)$.

Um algoritmo muito mais simples para o caso enraizado é apresentado por Holanda (2001). Este algoritmo computa em tempo $O(n \log n)$ uma árvore de busca cuja altura excede o ótimo por no máximo $\lg n$. Como a árvore ótima tem altura pelo menos $\lg n$, este algoritmo constitui uma 2-aproximação para o BOOP restrito a tais instâncias.

Com relação à aproximação no pior caso do problema geral, existe uma $O(\log n)$-aproximação polinomial, uma vez que pode-se facilmente checar que o BOOP é uma restrição do Problema da Árvore de Decisão para o qual um algoritmo guloso simples com tal razão de aproximação é conhecido (Arkin, Meijer, Mitchell, Rappaport, and Skiena, 1998). Algoritmos otimi- 
zados exponenciais para a construção de árvores de busca para ordens parciais são apresentados por Lipman and Abrahams (1995). Entretanto, este trabalho considera a minimização do comprimento dos caminhos ("path length") da árvore, em vez da sua altura.

A respeito do problema de Busca com Informação Parcial (BOIP), considerado no Capítulo 5, Linial and Saks (1985b) e Linial and Saks (1985a) determinam cotas para ordens arbitrárias e estudam em detalhe o caso no qual a ordem dada é um produto de cadeias e também o caso no qual o seu diagrama de Hasse é uma árvore enraizada. 


\section{Parte II}

\section{Consulta de Informação Cara em Bases de Dados}





\section{Preliminares}

Um hipergrafo é um par $G=(V(G), E(G))$ onde $V(G)$, o conjunto dos vértices de $G$, é um conjunto e cada aresta $e \in E(G)$ é um subconjunto não vazio de $V(G)$. Quando $v \in e \in E(G)$ dizemos que $v$ pertence a $e$, ou, equivalentemente, que $e$ incide em $v$.

A dimensão de $G$, denotada $r(G)$, é a cardinalidade da maior aresta de $G$. Um hipergrafo $G$ é dito $k$-uniforme se cada aresta tem exatamente $k$ vértices. Dizemos que $G$ é uniforme se é $k$-uniforme para algum $k>0$. Um grafo é um hipergrafo 2-uniforme.

Um hipergrafo $G$ é $k$-partido se existe uma partição de $V(G)$ em $k$ classes de vértices de forma que nenhuma aresta contenha mais do que um vértice da mesma classe. Uma partição assim será denotada $\left\{V_{i}(G): 1 \leq i \leq k\right\}$. No caso particular em que $k=2$, dizemos que $G$ é bipartido. O grafo bipartido $G$ é bipartido completo se $E(G)=\left\{\left\{v_{1}, v_{2}\right\}: v_{1} \in V_{1}(G), v_{2} \in V_{2}(G)\right\}$

A vizinhança de um vértice $v \in V(G)$ em $G$ é o conjunto

$$
\Gamma_{G}(v)=\{u \in V(G)-\{v\}:\{u, v\} \subseteq e \text { para algum } e \in E(G)\} .
$$

A idéia de vizinhança estende-se naturalmente para conjuntos de vértices, de modo que se $X \subseteq V(G)$, a vizinhança de $X$ em $G$ é

$$
\Gamma_{G}(X)=\bigcup_{v \in X} \Gamma_{G}(v)
$$

A estrela de um vértice $v$ é o conjunto de arestas incidentes em $v$, isto é, $\partial_{G}(v)=\{e \in E(G): v \in E\}$. O grau de $v$ em $G$ é a cardinalidade da estrela de $v$, isto é, $\operatorname{deg}_{G}(v)=\left|\partial_{G}(v)\right|$. Um vértice isolado de $G$ é um vértice de grau 0 em $G$.

Um emparelhamento em $G$ é um conjunto $M \subseteq E(G)$ cujas arestas são duas a duas disjuntas. Um hipergrafo $G$ é dito um emparelhamento se $E(G)$ é um emparelhamento. 
Uma cobertura de $G$ é um conjunto $C \subseteq V(G)$ que intercepta toda aresta de $G$. Dada uma função $\gamma: V(G) \rightarrow \mathbb{Z}$, uma cobertura mínima de $(G, \gamma)$ é uma cobertura $C$ de $G$ tal que $\sum_{v \in C} \gamma(v)$ é mínimo.

Dado um grafo $G$ e um conjunto de arestas $X \subseteq E(G)$, o subgrafo de $G$ gerado por $X$ é o grafo $G[X]$ dado por

$$
\begin{aligned}
& V(G[X])=\bigcup_{e \in X} e \\
& E(G[X])=X .
\end{aligned}
$$

Dados um grafo $G$ e uma função $f: V(G) \rightarrow \mathbb{N}$, dizemos que um conjunto de arestas $F \subseteq E(G)$ é um $f$-subfator de $G$ se, para todo $v \in V(G)$, o grau de $v$ em $G[F]$ está limitado superiormente por $f(v)$, isto é,

$$
\operatorname{deg}_{G[F]}(v) \leq f(v)
$$

Se vale a igualdade para cada $v \in V(G)$, dizemos que $F$ é um $f$-fator de $G$.

Omitimos o subscrito $G$ da notação sempre que o contexto permita fazêlo sem ambigüidade.

\section{A Técnica Minimax de Yao}

Teorema 1. Sejam $\mathcal{A}$ e $\mathcal{I}$ dois espaços de probabilidades de suporte finito. Para toda função $f: \mathcal{A} \times \mathcal{I} \rightarrow \mathbb{R}$,

$$
\min \left\{\mathbb{E}_{\mathcal{I}}[f(A, I)]: A \in \mathcal{A}\right\} \leq \max \left\{\mathbb{E}_{\mathcal{A}}[f(A, I)]: I \in \mathcal{I}\right\}
$$

Demonstração. Seja $\left\{A_{1}, \ldots, A_{n}\right\}$ o conjunto dos elementos de $\mathcal{A}$ com probabilidade positiva e seja $\pi$ o vetor de dimensão $n$ dado por $\pi_{i}=\mathbb{P}_{\mathcal{A}}\left(A_{i}\right)$, $1 \leq i \leq n$. De modo análogo, seja $\left\{I_{1}, \ldots, I_{m}\right\}$ o conjunto dos elementos de $\mathcal{I}$ com probabilidade positiva e seja $\sigma$ o vetor de dimensão $m$ dado por $\sigma_{j}=\mathbb{P}_{\mathcal{I}}\left(I_{j}\right), 1 \leq j \leq m$. Finalmente, seja $M$ a matriz de dimensões $m \times n$ dada por $M_{i}^{j}=f\left(A_{i}, I_{j}\right)$.

Para cada $1 \leq i \leq n$ temos

$$
\mathbb{E}_{\mathcal{I}}\left[f\left(A_{i}, I\right)\right]=\sum_{j=1}^{m} f\left(A_{i}, I_{j}\right) \mathbb{P}_{\mathcal{I}}\left(I_{j}\right)=\sum_{j=1}^{m} M_{i}^{j} \sigma_{j}=(M \sigma)_{i}
$$


ou seja, para cada $1 \leq i \leq n$, a média $\mathbb{E}_{\mathcal{I}}\left[f\left(A_{i}, I\right)\right]$ é uma variável aleatória no espaço de probabilidades $\mathcal{A}$, cujo valor esperado, por sua vez é

$$
\begin{aligned}
& \mathbb{E}_{\mathcal{A}}\left[\mathbb{E}_{\mathcal{I}}\left[f\left(A_{i}, I\right)\right]\right]=\sum_{i=1}^{n} \mathbb{E}_{\mathcal{I}}\left[f\left(A_{i}, I\right)\right] \mathbb{P}_{\mathcal{A}}\left(A_{i}\right) \\
&=\sum_{i=1}^{n}(M \sigma)_{i} \pi_{i}=\pi(M \sigma)=\pi M \sigma
\end{aligned}
$$

Como o valor mínimo de uma variável aleatória não pode ser maior que sua média, temos

$$
\min \left\{\mathbb{E}_{\mathcal{I}}\left[f\left(A_{i}, I\right)\right]: 1 \leq i \leq n\right\} \leq \mathbb{E}_{\mathcal{A}}\left[\mathbb{E}_{\mathcal{I}}\left[f\left(A_{i}, I\right)\right]\right],
$$

ou, equivalentemente

$$
\min \left\{\mathbb{E}_{\mathcal{I}}\left[f\left(A_{i}, I\right)\right]: 1 \leq i \leq n\right\} \leq \pi M \sigma,
$$

e, conseqüentemente

$$
\min \left\{\mathbb{E}_{\mathcal{I}}[f(A, I)]: A \in \mathcal{A}\right\} \leq \pi M \sigma,
$$

Um argumento em tudo análogo permite concluir

$$
\pi M \sigma \leq \max \left\{\mathbb{E}_{\mathcal{A}}[f(A, I)]: I \in \mathcal{I}\right\},
$$

e daí

$$
\min \left\{\mathbb{E}_{\mathcal{I}}[f(A, I)]: A \in \mathcal{A}\right\} \leq \max \left\{\mathbb{E}_{\mathcal{A}}[f(A, I)]: I \in \mathcal{I}\right\}
$$

Considere um problema computacional $\mathcal{P}$ e seja $\mathcal{A}$ um espaço de probabilidades cujos eventos elementares são os algoritmos determinísticos para $\mathcal{P}$. Um algoritmo aleatorizado $\mathcal{R}$ para o problema $\mathcal{P}$ pode ser concebido como um algoritmo determinístico que recebe como entrada, além de uma instância $I$ do problema $\mathcal{P}$, uma seqüência finita de "bits aleatórios" $x$. Uma vez fixada a seqüência de "bits aleatórios" $x$ utilizados numa execução de $\mathcal{R}$, então $\mathcal{R}$ reduz-se a algum dos algoritmos em $\mathcal{A}$, no sentido em que para toda instância $I$ de $\mathcal{P}, \mathcal{R}(I, x)=A(I)$. O mesmo dito em outras palavras é afirmar que cada distribuição de probabilidades de suporte finito de $\mathcal{A}$ corresponde a um possível algoritmo aleatorizado para $\mathcal{P}$. Com isso, podemos especializar o Teorema 1 (página 54) nos seguintes termos. 
Teorema 2. Seja $\mathcal{P}$ um problema computacional de contra-domínio $\mathcal{D}$ e seja I um espaço de probabilidades de suporte finito cujos eventos elementares são instâncias de $\mathcal{P}$. Seja ainda $g: \mathcal{D} \rightarrow \mathbb{R}$. Então, para todo algoritmo aleatorizado $\mathcal{R}$ para $\mathcal{P}$ vale que

$\min \left\{\mathbb{E}_{\mathcal{I}}[g(\mathcal{A})]: \mathcal{A}\right.$ é um algoritmo determinístico para $\left.\mathcal{P}\right\}$

$$
\leq \max \{\mathbb{E}[g(\mathcal{R}(I))]: \text { I é uma instância de } \mathcal{P}\} .
$$

O resultado a seguir é uma aplicação pouco usual do Teorema 2, que será usada na prova do Corolário 2.18 , um resultado onde provamos a impossibilidade de se obter bom desempenho para um certo tipo de algoritmo com alta probabilidade.

Corolário 3. Seja $\mathcal{P}$ um problema computacional de contra-domínio $\mathcal{D} e$ seja I um espaço de probabilidades de suporte finito cujos eventos elementares são instâncias de $\mathcal{P}$. Seja ainda $P$ um predicado sobre $\mathcal{D}$. Então, para todo algoritmo aleatorizado $\mathcal{R}$ para $\mathcal{P}$ vale que

$$
\begin{aligned}
\min \left\{\mathbb{P}_{\mathcal{I}}(P(A(I))): \mathcal{A} \text { é um algoritmo determinístico para } \mathcal{P}\right\} \\
\quad \leq \max \{\mathbb{P}[P(\mathcal{R}(I))]: \text { I é uma instância de } \mathcal{P}\} .
\end{aligned}
$$

Demonstração. Basta tomar, no Teorema 2, $g: \mathcal{D} \rightarrow\{0,1\}$ dada por $g(A(I))=1$ se $P(A(I))$ é satisfeito e $g(A(I))=0$ caso contrário. Nesse caso temos que $\mathbb{E}_{\mathcal{I}}[g(A(I))]=\mathbb{P}_{\mathcal{I}}(P(A(I)))$ e $\mathbb{E}[g(\mathcal{R}(I))]=\mathbb{P}(P(\mathcal{R}(I)))$ e basta reescrever (1) nesses termos. 


\section{Introdução}

O projeto e implementação de um Sistema Gerenciador de Base de Dados (SGBD) é uma tarefa complexa, que envolve a abordagem de muitos problemas computacionais diferentes. Um destes problemas é conhecido na literatura especializada da área como o problema de Otimização de Consultas. Nesta parte do trabalho abordamos um problema cuja motivação original surge no contexto do problema de Otimização de Consultas de SGBDs que apresentam certas características não convencionais.

Do ponto de vista do usuário final, um SGBD é um sistema que armazena informações e responde a consultas a respeito dessas informações. De maneira esquemática o processo pode ser descrito da seguinte maneira. $\mathrm{O}$ usuário formula uma consulta em uma linguagem de alto nível e um interpretador dessa linguagem traduz a consulta feita para uma representação intermediária. O problema de Otimização de Consultas de SGBDs consiste em, a partir da expressão da consulta nessa linguagem intermediária, obter uma expressão semanticamente equivalente cuja avaliação seja a menos custosa possível. Essa versão otimizada é que então será submetida à avaliação pelo avaliador de consultas do SGBD.

No Modelo Relacional de bases de dados concebe-se cada unidade de informação como uma $k$-upla $e \in V_{1} \times V_{2} \times \ldots \times V_{k}$, onde $V_{1}, V_{2}, \ldots, V_{k}$ são conjuntos. Essas $k$-uplas são usualmente chamadas de tuplas da relação e um conjunto de tuplas tais é chamado de uma relação. Uma Base de Dados Relacional pode ser entendida, portanto, como um conjunto de relações.

Uma das operações primitivas do repertório do avaliador de consultas de um Sistema Gerenciador de Bases de Dados Relacional (SGBDR) é a operação conhecida como seleção. Uma seleção é uma operação binária cujos operandos são uma relação e um predicado. A seleção de uma relação $R$ por um predicado $P$ é usualmente denotada por $\sigma_{P}(R)$ e seu resultado consiste na relação formada pelas tuplas da relação $R$ que satisfazem o predicado $P$, 
isto é, $\sigma_{P}(R)=\{e \in R: P(e)$ é satisfeito $\}$.

Uma presunção implícita no processo de otimização de consultas de muitos SGBDRs com relação ao custo de avaliação de uma seleção $\sigma_{P}(R)$ é a de que não existe diferença significativa entre os custos de avaliação de $P(e)$ para as diferentes tuplas $e \in R$. Noutras palavras, fixado um predicado $P$ e dadas duas tuplas $e_{1}, e_{2} \in R$, assume-se que a diferença entre os custos de avaliação de $P\left(e_{1}\right)$ e $P\left(e_{2}\right)$ é desprezível. Dando tal premissa por assente, a avaliação de uma seleção $\sigma_{P}(R)$ por um SGBDR "sempre tem o mesmo custo", qualquer que seja a ordem em que o predicado $P$ seja avaliado sobre as tuplas de $R$.

Nesta parte do trabalho tratamos do problema de otimização que emerge quando a presunção descrita acima não é válida, isto é, quando a ordem de avaliação de um predicado $P$ sobre as tuplas de uma relação $R$ tem um impacto não desprezível no custo global de avaliação de uma seleção $\sigma_{P}(R)$. Este é o caso de SGBDRs cujas relações podem conter informação massiva como imagens, seqüências de texto arbitrariamente longas (por exemplo, representações de seqüencias de DNA), planilhas de cálculo, seqüências de som ou vídeo digitalizado, grandes arquivos de texto etc. Em casos assim, a avaliação de um predicado sobre uma tupla de uma relação pode envolver processamento cujo tempo varia significativamente de tupla para tupla. Conseqüentemente, a ordem de avaliação do predicado sobre as tuplas de uma relação numa operação de seleção pode fazer uma grande diferença no que diz respeito ao tempo de resposta às consultas do SGBDR.

A título de ilustração, considere o exemplo onde temos uma relação $R=\left\{e_{1}=\left(v_{1}, v_{3}\right), e_{2}=\left(v_{1}, v_{4}\right), e_{3}=\left(v_{1}, v_{5}\right), e_{4}=\left(v_{2}, v_{3}\right)\right\}$, conforme ilustrado na Figura 1, e suponha que deseja-se avaliar a seleção $\sigma_{P}(R)$ onde o predicado $P$ é dado por $P(e)=P_{1}\left(v_{i}\right) \wedge P_{2}\left(v_{j}\right)$ para cada $e=\left(v_{i}, v_{j}\right) \in R$, sendo $P_{1}$ e $P_{2}$ predicados de domínios $\left\{v_{1}, v_{2}\right\}$ e $\left\{v_{3}, v_{4}, v_{5}\right\}$, respectivamente. Assumamos que $P_{1}\left(v_{1}\right)$ seja verdadeiro e que o custo de avaliação de $P_{1}\left(v_{1}\right)$ seja 3 ; que $P_{2}(v)$ seja falso para todo $v \in\left\{v_{3}, v_{4}, v_{5}\right\}$ e que o custo de avaliação de $P_{2}(v)$ seja 2 para todo $v \in\left\{v_{3}, v_{4}, v_{5}\right\}$. Neste caso, se a avaliação de $\sigma_{P}(R)$ começa por $P_{1}\left(v_{1}\right)$, então essa avaliação terá custo pelo menos 9 , pois será inevitável avaliar $P_{2}\left(v_{3}\right), P_{2}\left(v_{4}\right)$ e $P_{2}\left(v_{5}\right)$ para concluir que $\sigma_{P}(R)=\emptyset$. Entretanto, para chegar à mesma conclusão, bastaria avaliar $P_{2}(v)$ para cada $v \in\left\{v_{3}, v_{4}, v_{5}\right\}$, o que teria custo 6 .

Este exemplo ilustra os elementos essenciais do problema que consideramos. São dados $k$ conjuntos disjuntos $V_{1}, V_{2}, \ldots, V_{k}$, uma relação $R \subseteq V_{1} \times V_{2} \times \ldots \times V_{k}$, um predicado $P=P_{1} \wedge P_{2} \wedge \ldots \wedge P_{k}$, onde $P_{i}$ é um predicado de domínio $V_{i}$ para cada $1 \leq i \leq k$. A cada $v \in V_{i}$, 


\begin{tabular}{l|c|c|}
$e_{1}:$ & $v_{1}$ & $v_{3}$ \\
\cline { 2 - 3 }$e_{2}:$ & $v_{1}$ & $v_{4}$ \\
\cline { 2 - 3 }$e_{3}:$ & $v_{1}$ & $v_{5}$ \\
\cline { 2 - 3 }$e_{4}:$ & $v_{2}$ & $v_{3}$ \\
\cline { 2 - 3 } & &
\end{tabular}

Figura 1: A relação $R=\left\{\left(v_{1}, v_{3}\right),\left(v_{1}, v_{4}\right),\left(v_{1}, v_{5}\right),\left(v_{2}, v_{3}\right)\right\}$

$1 \leq i \leq k$, está associado um custo para a avaliação de $P_{i}(v)$. Desejamos determinar um conjunto $\mathrm{A} \subseteq V_{1} \cup V_{2} \cup \ldots \cup V_{k}$ tal que a avaliação dos predicados $P_{i}(v), 1 \leq i \leq k$ para cada $v \in$ A permita avaliar $P(e)$ para todo $e \in R$ com o menor custo possível.

Estudamos duas variantes deste problema, que chamamos de seqüencial e distribuída. A variante seqüencial corresponde ao cenário em que um único processador é responsável pela avaliação dos predicados $P_{i}(v), v \in V_{i}$, $1 \leq i \leq k$. A variante distribuída corresponde ao cenário em que existem $k$ processadores disponíveis, cada um dedicado à avaliação de cada predicado $P_{i}, 1 \leq i \leq k$. Na variante distribuída, consideramos dois modelos de computação, o preemptivo e o não preemptivo, conforme cada processador possa ou não interromper (e posteriormente reempreender) a avaliação de $P_{i}(v), v \in V_{i}, 1 \leq i \leq k$, respectivamente.

\section{Formalização do Problema}

Formalizaremos o problema da seguinte maneira. A relação $R$ será representada por um hipergrafo $G$ dado por $V(G)=V_{1} \cup V_{2} \cup \ldots \cup V_{k}$ e $E(G)=\left\{\left\{v_{1}, \ldots, v_{k}\right\} \subseteq V(G):\left(v_{1}, \ldots, v_{k}\right) \in R\right\}$. O hipergrafo $G$, portanto, é $k$-partido, $k$-uniforme e não tem vértices isolados. Uma função $\gamma: V(G) \rightarrow \mathbb{Z}$ indicará para cada $v \in V_{i}(G), 1 \leq i \leq k$, o custo $\gamma(v)$ da avaliação do predicado $P_{i}(v)$. Finalmente, uma função $\delta: V(G) \rightarrow\{0,1\}$ indicará o valor verdade do predicado $P_{i}(v)$ para cada $v \in V_{i}(G), 1 \leq i \leq k$.

Dada uma tripla $(G, \delta, \gamma)$ onde $G, \delta$ e $\gamma$ são como acima, definimos a função $\bar{\delta}(e): E(G) \rightarrow\{0,1\}$ por

$$
\bar{\delta}(e)=\prod_{v \in e} \delta(v) .
$$

Assim, se cada aresta $e \in E(G)$ representa uma tupla da relação $R$, então $\bar{\delta}(e)$ vale 1 ou 0 conforme $P(e)$ seja ou não satisfeito.

Nesta parte do trabalho diremos que um conjunto $\mathrm{A} \subseteq V(G)$ é uma 
avaliação de $(G, \delta, \gamma)$ se o conhecimento do valor de $\delta(v)$ para cada vértice $v \in$ A permite determinar o valor de $\bar{\delta}(e)$ para toda aresta $e \in E(G)$. Antes de prosseguir, entretanto, é importante ressaltar que para o problema motivador original, especialmente em sua variante distribuída, uma avaliação é na verdade um objeto mais complexo do que simplesmente um conjunto de vértices, devido à informação relativa ao escalonamento (ordem de avaliação dos predicados). Para ilustrar o tipo de questões que surgem em torno desse escalonamento, considere uma instância $(G, \delta, \gamma)$ do OD2d, na qual $G$ tem uma única aresta $\left\{v_{1}, v_{2}\right\}$ com custos $\gamma\left(v_{1}\right)=1$ e $\gamma\left(v_{2}\right)=1000$.

1. no modelo não-preemptivo, se ambos processadores começam a avaliar simultaneamente, o custo seria 1000 , quaisquer que sejam os valores de $\delta\left(v_{1}\right)$ e $\delta\left(v_{2}\right)$; se o processador a cargo de $V_{2}(G)$ espera pelo resultado da avaliação de $v_{1}$ antes de processar $v_{2}$, o custo da avaliação seria 1001 , se $\delta\left(v_{1}\right)=1$, ou 1 , se $\delta\left(v_{1}\right)=0$.

2. no modelo preemptivo o custo seria 1 , se $\delta\left(v_{1}\right)=0$, ou 1000 , se $\delta\left(v_{1}\right)=1$.

Por sutis que pareçam estas questões à primeira vista, o fato é que o tipo de resultados em que estamos interessados não exige formalizar explicitamente a informação relativa ao escalonamento contida numa avaliação. A fim de não carregar desnecessariamente o texto com a distinção entre uma avaliação propriamente dita e os vértices que a compõem, dada uma avaliação A, definimos o custo $\gamma(\mathrm{A})$ da avaliação A como sendo o tempo decorrido entre o início da avaliação pelo primeiro processador e o fim da avaliação pelo último processador.

Dada uma instância $\mathcal{I}=(G, \delta, \gamma)$ do problema, seja $X \subseteq V(G)$. Definimos o custo seqüencial de $X$ e o custo seqüencial mínimo de $\mathcal{I}$ por

$$
\begin{aligned}
& \gamma_{\mathbf{s}}(X)=\sum_{v \in X} \gamma(v), \\
& \gamma_{\mathbf{s}}^{*}(\mathcal{I})=\min \left\{\gamma_{\mathbf{s}}(\mathrm{A}): \text { A é uma avaliação de } \mathcal{I}\right\} .
\end{aligned}
$$

Do mesmo modo, definimos o custo distribuído de $X$ e o custo distribuído mínimo de $\mathcal{I}$ por

$$
\begin{aligned}
\gamma_{\mathrm{d}}(X) & =\max \left\{\gamma_{\mathrm{s}}\left(X \cap V_{i}(G)\right): 1 \leq i \leq k\right\}, \\
\gamma_{\mathrm{d}}^{*}(\mathcal{I}) & =\min \left\{\gamma_{\mathrm{d}}(\mathrm{A}): \text { A é uma avaliação de } \mathcal{I}\right\} .
\end{aligned}
$$

Assim, dada uma avaliação propriamente dita A de uma instância do problema e denotando por $V(\mathrm{~A})$ os vértices que a compõem, temos que, na 
variante seqüencial,

$$
\gamma(\mathrm{A})=\gamma_{\mathrm{s}}(V(\mathrm{~A}))
$$

enquanto que na variante distribuída,

$$
\gamma(\mathrm{A}) \geq \gamma_{\mathbf{d}}(V(\mathrm{~A})) .
$$

Uma vez feito este esclarecimento, poderemos permitir-nos o abuso de, daqui por diante, tratar uma avaliação de uma instância $(G, \delta, \gamma)$ do problema como um conjunto de vértices de $G$, em favor da fluência de leitura, simplificando a notação e evitando a cada ponto ter que fazer a distinção entre uma "avaliação A propriamente dita" e "o conjunto de vértices $V(\mathrm{~A})$ que compõe a avaliação". Podemos por fim apresentar a definição do problema em termos mais formais. Para cada $k>0$ temos a seguinte definição.

\section{Problema: Ordenação Dinâmica $k$-partida (ODk)}

Entrada: Uma tripla $(G, \delta, \gamma)$ onde $G$ é um hipergrafo $k$-partido, $k$-uniforme, sem vértices isolados, $\gamma: V(G) \rightarrow \mathbb{Z}$ e $\delta: V(G) \rightarrow\{0,1\}$.

Saída: Uma avaliação custo mínimo de $(G, \delta, \gamma)$.

A variante seqüencial do problema será chamada de problema de Ordenação Dinâmica $k$-partida Seqüencial (ODks) e a variante distribuída de problema de Ordenação Dinâmica $k$-partida Distribuída (OD $k$ d). Com o risco de abusar da paciência do leitor, chamamos novamente a atenção para o fato de que o que distingüe uma variante do problema da outra é a definição do custo $\gamma(\mathrm{A})$ de uma avaliação $\mathrm{A}$, para o qual não apresentamos uma descrição analítica, somente a definição de que "representa o tempo decorrido entre o início da avaliação pelo primeiro processador e o fim da avaliação pelo último processador". Na verdade, para o ODks, a igualdade em (2) representa efetivamente o valor de $\gamma(\mathrm{A})$. No caso do OD $k$ d, entretanto, será suficiente para nossos propósitos a desigualdade (3). Como boa parte da discussão pelo resto deste capítulo aplica-se a ambas as variantes (seqüencial e distribuída) do problema, usamos OD $k$ como uma maneira conveniente de referir-nos conjuntamente a OD $k$ s e OD $k \mathrm{~d}$.

Dada uma instância $(G, \delta, \gamma)$ do $\mathrm{OD} k$, diremos que o vértice $v \in V(G)$ é verdadeiro se $\delta(v)=1$ e diremos que $v$ é falso se $\delta(v)=0$. Diremos que uma aresta $e \in E(G)$ é verdadeira se todos os seus vértices são verdadeiros, isto é, se $\bar{\delta}(e)=1$, e que é falsa se algum de seus vértices é falso, ou seja, se $\bar{\delta}(e)=0$. Feitas estas definições, pode ser esclarecedor a essa altura da discussão enunciar os seguintes resultados imediatos. 
Lema 4. Dada uma instância $\mathcal{I}=(G, \delta, \gamma)$ do $\mathrm{OD} k$, um conjunto $\mathrm{A} \subseteq V(G)$ é uma avaliação de $\mathcal{I}$ se e somente se contém todos os vértices de todas as arestas verdadeiras de $G$, e um vértice falso de cada aresta falsa de $G$.

Corolário 5. Toda avaliação de uma instância $(G, \delta, \gamma)$ do ODk é uma cobertura de $G$.

Dada uma instância $(G, \delta, \gamma)$ do $\mathrm{OD} k$, vamos denotar por $V^{0}(G)$ e por $V^{1}(G)$ os conjuntos de vértices falsos e verdadeiros de $G$, respectivamente. Do mesmo modo, vamos denotar por $E^{0}(G)$ e por $E^{1}(G)$ os conjuntos de arestas falsas e verdadeiras de $G$. Finalmente, vamos denotar por $G^{0}$ e por $G^{1}$ os subgrafos de $G$ gerados por $E^{0}(G)$ e por $E^{1}(G)$, e vamos denotar por $\Gamma^{1}(X)$ o conjunto dos vizinhos dos vértices verdadeiros de $X \subseteq V(G)$, isto é,

$$
\begin{aligned}
V^{0}(G) & =\delta^{-1}(0)=\{v \in V(G): \delta(v)=0\}, \\
V^{1}(G) & =\delta^{-1}(1)=\{v \in V(G): \delta(v)=1\}, \\
E^{0}(G) & =\bar{\delta}^{-1}(0)=\{e \in E(G): \bar{\delta}(e)=0\}, \\
E^{1}(G) & =\bar{\delta}^{-1}(1)=\{e \in E(G): \delta(e)=1\}, \\
G^{0} & =G\left[E^{0}(G)\right], \\
G^{1} & =G\left[E^{1}(G)\right], \\
\Gamma^{1}(X) & =\Gamma\left(X \cap V^{1}(G)\right) .
\end{aligned}
$$

Num esforço por tornar mais nítidos os contornos do problema, enunciamos as seguintes conseqüências imediatas do Lema 4 (página 62).

Corolário 6. Uma avaliação ótima para uma instância $(G, \delta, \gamma)$ do ODks é dada por $V\left(G^{1}\right) \cup C^{0}$, onde $C^{0}$ é uma cobertura de $G^{0}$ composta somente por vértices de $V^{0}(G)$ e de custo seqüencial mínimo.

Corolário 7. Uma avaliação ótima para uma instância $(G, \delta, \gamma)$ do ODkd é dada por $V\left(G^{1}\right) \cup C^{0}$, onde $C^{0}$ é uma cobertura de $G^{0}$ composta somente por vértices de $V^{0}(G)$ e de custo distribuído mínimo.

\section{Medida Comparativa de Desempenho}

A comparação entre diferentes algoritmos para o $\mathrm{OD} k$ não é uma questão trivial. O OD $k$ pertence à classe do que se convencionou chamar problemas de computação "on-line", ou seja, problemas cujos algoritmos não tem 
acesso ao conjunto completo de dados de entrada ao início da computação e, portanto, precisam "tomar decisões" que afetam o resultado final baseados no "conhecimento parcial" da entrada.

Uma proposta de medida comparativa para o desempenho de algoritmos "on-line" é a chamada análise competitiva de algoritmos, que será utilizada nesta parte do trabalho na análise de algoritmos para o OD $k$. Em breves palavras, podemos dizer que a análise competitiva de algoritmos consiste em medir a eficiência de um algoritmo para um problema "on-line" comparando seu resultado com o melhor resultado possível de um algoritmo para a versão "off-line" do mesmo problema, isto é, um algoritmo que tem, desde o início da computação, acesso completo à entrada. Essa medida é dada pela razão entre os resultados dos algoritmos "on-line" e "off-line" cada um deles expressos sob a forma de uma quantidade. Essa razão é chamada (razão de) competitividade do algoritmo.

É interessante notar que, por definição, a competitividade de um algoritmo "on-line" é um número maior ou igual a 1 e que, segundo essa medida, um algoritmo será considerado tão mais competititivo ("melhor") quanto menor (mais próxima de 1) seja sua competitividade. Para evitar a dificuldade de leitura ocasionada por asserções como "um algoritmo maximamente competitivo é aquele que tem competitividade mínima", ao longo do texto procuramos usar adjetivos como "ótimo", "melhor" e "pior" para referir-nos à competitividade, em vez de "máximo", "mínimo", "maior" ou "menor". O exemplo acima, por exemplo, seria fraseado como "um algoritmo otimamente competitivo é aquele que tem a melhor competitividade possível".

Os Corolários 6 e 7 (página 62) mostram que o conjunto de vértices de uma avaliação ótima para o OD $k$ é bem caracterizado e computável. Por isso, a fim de simplificar a discussão, especializamos a definição de razão de competitividade e suas correlatas nesta parte do trabalho, referindo-as diretamente à solução ótima do problema e sem referência a um hipotético algoritmo "off-line".

Dada uma instância $\mathcal{I}=(G, \delta, \gamma)$ do $\mathrm{OD} k$, vamos denotar por $\gamma^{*}(\mathcal{I})$ o custo de uma avaliação ótima de $\mathcal{I}$. Sem perda de generalidade, assumimos ao longo de todo o texto que $\gamma^{*}(\mathcal{I})>0$. Note que, de acordo com (2) e (3) temos $\gamma^{*}(\mathcal{I})=\gamma_{\mathrm{s}}^{*}(\mathcal{I})$ no OD $k$ s e $\gamma^{*}(\mathcal{I}) \geq \gamma_{\mathrm{d}}^{*}(\mathcal{I})$ no OD $k \mathrm{~d}$.

Dada uma avaliação $A$ de $\mathcal{I}$, definimos a competitividade de $\mathrm{A}$ como a razão

$$
c(\mathrm{~A}, \mathcal{I})=\frac{\gamma(\mathrm{A})}{\gamma^{*}(\mathcal{I})}
$$


Dado um algoritmo $\mathcal{A}$ para o $\mathrm{OD} k$, denotamos por $\mathcal{A}(\mathcal{I})$ a avaliação de $\mathcal{I}$ computada por $\mathcal{A}$ e definimos a competitividade de $\mathcal{A}$ como o pior valor atingido pela competitividade da avaliação $\mathcal{A}(\mathcal{I})$ consideradas todas as instâncias $\mathcal{I}$ do problema, isto é

$$
\begin{aligned}
& c(\mathcal{A})=\max \{c(\mathcal{A}(\mathcal{I}), \mathcal{I}): \mathcal{I} \text { é uma instância do OD } k\} \\
& =\max \left\{\frac{\gamma(\mathcal{A}(\mathcal{I}))}{\gamma^{*}(\mathcal{I})}: \mathcal{I} \text { é uma instância do OD } k\right\} .
\end{aligned}
$$

Quando $\mathcal{A}$ é um algoritmo aleatorizado, o custo $\gamma(\mathcal{A}(\mathcal{I}))$ da avaliação $\mathcal{A}(\mathcal{I})$ e, conseqüentemente, sua competitividade $c(\mathcal{A}(\mathcal{I}), \mathcal{I})$, são variáveis aleatórias. Nesse caso, definimos a competitividade de $\mathcal{A}$ em função do valor esperado de $c(\mathcal{A}(\mathcal{I}), \mathcal{I})$ para cada instância $\mathcal{I}$, isto é,

$$
\begin{aligned}
c(\mathcal{A})=\max \{\mathbb{E}[c(\mathcal{A}(\mathcal{I}), \mathcal{I})]: \mathcal{I} \text { é uma instância do OD } k\} \\
=\max \left\{\mathbb{E}\left[\frac{\gamma(\mathcal{A}(\mathcal{I}))}{\gamma^{*}(\mathcal{I})}\right]: \mathcal{I} \text { é uma instância do OD } k\right\} \\
=\max \left\{\frac{\mathbb{E}[\gamma(\mathcal{A}(\mathcal{I}))]}{\gamma^{*}(\mathcal{I})}: \mathcal{I} \text { é uma instância do OD } k\right\} .
\end{aligned}
$$

\section{Organização do Texto}

O foco desta parte do trabalho é a determinação de algoritmos eficientes e competitivos para o OD $k$.

No Capítulo 1 apresentamos formulações do OD $k$ como jogo de esconder e provamos alguns resultados que serão utilizados no estabelecimento de cotas mínimas para a competitividade de algoritmos para o OD $k$ nos capítulos subseqüentes.

O Capítulo 2 trata da variante seqüencial do problema. Estabelecemos cotas para a competitividade de algoritmos determinísticos e aleatorizados para o ODks e apresentamos um algoritmo polinomial determinístico otimamente competitivo para o ODks. Estudamos mais detidamente o caso particular do OD $k$ s em que $k=2$ (OD2s). Apresentamos um algoritmo aleatorizado polinomial para o OD2s de competitividade $1+\sqrt{2} / 2$ e provamos que, dado $\epsilon>0$ uma avaliação computada por um algoritmo aleatorizado para o OD2s não pode ter competitividade melhor que $1+\epsilon$ com probabilidade maior do que $(1+\epsilon) / 2$ para todas as instâncias.

O Capítulo 3 trata da variante distribuída do problema. Estabelecemos cotas para a competitividade de algoritmos determinísticos para o ODkd, 
tanto no modelo preemptivo como no não-preemptivo. Apresentamos um algoritmo polinomial otimamente competitivo para o OD $k$ d segundo o modelo preemptivo e um algoritmo otimamente competitivo para o OD $k \mathrm{~d}$ segundo o modelo não-preemptivo.

O Capítulo 4 dedica-se a provar o Teorema 4.6. Trata-se de um resultado a respeito de uma das formulações do OD $k$ como jogo de esconder apresentadas no Capítulo 1. Esta demonstração apresenta algum interesse, independente do contexto do estudo do OD $k$, na medida em que trata de um conceito correlato do conceito de "evasividade de propriedades" para um grafo bipartido.

No Capítulo 5 apresentamos uma breve resenha da literatura a respeito do $\mathrm{OD} k$ e alguns problemas correlatos, indicamos problemas em aberto, e fazemos alguns comentários adicionais.

\section{Créditos}

Este trabalho foi realizado em conjunto com Tomás Feder (Computer Science Department, Stanford University), Yoshiharu Kohayakawa (Departamento de Ciência da Computação do IME-USP), Eduardo Laber (Departamento de Informática da PUC-RJ), Rajeev Motwani (Computer Science Department, Stanford University), Liadan O'Callaghan (Computer Science Department, Stanford University), Rina Panigrahy (Cisco Systems) e Dilys Thomas (Computer Science Department, Stanford University).

Uma versão preliminar deste trabalho, elaborada em conjunto com Eduardo Laber (Departamento de Informática da PUC-RJ) e Yoshiharu Kohayakawa (Departamento de Ciência da Computação do IME-USP) foi publicada como "Resumo Estendido" na edição de 2004 do Latin American Theoretical INformatics (LATIN 2002) (Laber, Carmo, and Kohayakawa, 2004). A partir desta publicação entraram em contato conosco os demais autores acima citados que trabalhavam independentemente no problema. O resultado dessa interação, que reúne o trabalho de ambos os grupos, foi submetido para publicação no ACM Transactions on Algorithms, onde encontra-se em fase de preparação do manuscrito final.

O conteúdo do Capítulo 4 foi publicado em Carmo, Feder, Kohayakawa, Laber, Motwani, O'Callaghan, Panigrahy, and Thomas (2004b) e alguns dos resultados dos demais capítulos foram publicados em Laber, Carmo, and Kohayakawa (2003). 
Durante a elaboração deste trabalho, entramos em contato com o Grupo de Biologia Computacional do IME-USP a fim de estudar a possibilidade de implementação de protótipos dos algoritmos aqui apresentados às suas bases de dados. O autor agradece a disponibilidade e acolhimento por parte dos membros deste grupo, e muito em especial, do Prof. João Eduardo Ferreira e de Gustavo Tadao Okida. 


\section{Capítulo 1}

\section{Ordenação Dinâmica $k$-partida como Jogo de Esconder}

Neste capítulo apresentamos duas formulações do OD $k$ como jogo de esconder. A primeira delas, o jogo $\mathcal{J}$, é uma versão direta do $O D k$ em jogo de esconder. A outra, o jogo $\mathcal{B}$, é uma versão especializada para as instâncias $(G, \delta, \gamma)$ do OD $k$ onde $G$ é um emparelhamento. Os resultados deste capítulo serão utilizados no estabelecimento de cotas para a competitividade de algoritmos para o OD $k$ apresentadas nos capítulos subseqüentes.

Iniciamos por definir o jogo $\mathcal{J}$.

Jogo: Ordenação Dinâmica $k$-partida $(\mathcal{J}(G, \gamma))$

Início: os jogadores recebem um hipergrafo $k$-partido, $k$-regular e sem vértices isolados $G$ e uma função $\gamma: V(G) \rightarrow \mathbb{Z}$; a ocultadora escolhe uma função $\delta: V(G) \rightarrow\{0,1\}$.

Lance: cada pergunta é um vértice $v \in V(G)$; a resposta é o valor de $\delta(v)$.

Fim: o procurador encontra-se em condições de determinar o valor de $\bar{\delta}(e)$ para toda aresta $e \in E(G)$.

Dada uma realização $\mathcal{R}=\left(\left(v_{1}, a_{1}\right), \ldots,\left(v_{n}, a_{n}\right)\right)$ do jogo $\mathcal{J}(G, \gamma)$, definimos o custo de $\mathcal{R}$ como $\gamma(\mathcal{R})=\gamma\left(\left\{v_{1}, v_{2}, \ldots, v_{n}\right\}\right)$. O OD $k$ é, portanto, um problema de busca ótima nos moldes da discussão da Seção 2. A diferença 
é que aqui a duração de uma realização do jogo corresponde não ao número de jogadas, mas ao custo do conjunto de jogadas que, tanto no ODks como no OD $k$ d, é uma função crescente de $2^{V(G)}$.

Considere a estratégia de evasão $\mathcal{E}$ para o jogo $\mathcal{J}(G, \gamma)$ que consiste em responder 1 a toda pergunta $v$, exceto quando $v$ é o último vértice não avaliado de alguma aresta de $G$.

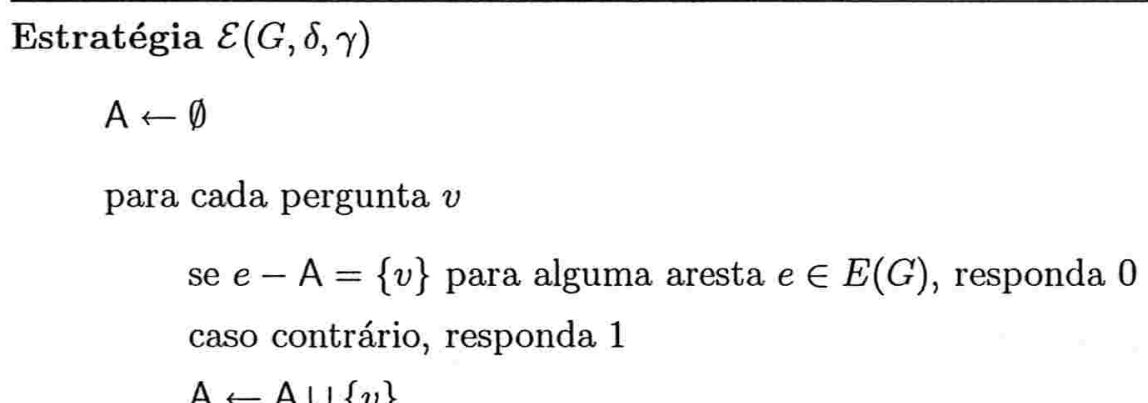

$\mathrm{A} \leftarrow \mathrm{A} \cup\{v\}$

A estratégia de evasão $\mathcal{E}$ permite enunciar o seguinte resultado.

Lema 1.1. Todo algoritmo deterministico $\mathcal{A}$ para o $\mathrm{OD} k$ admite uma instância $\mathcal{I}_{\mathcal{A}}=\left(G_{\mathcal{A}}, \delta_{\mathcal{A}}, \gamma_{\mathcal{A}}\right)$ tal que $\mathcal{A}\left(\mathcal{I}_{\mathcal{A}}\right)=V\left(G_{\mathcal{A}}\right)$.

Demonstração. Seja $\mathcal{A}$ um algoritmo determinístico para o ODk, e seja $\mathcal{E}_{\mathcal{A}}$ a estratégia de busca para o jogo $\mathcal{J}$ correspondente ao Algoritmo $\mathcal{A}$.

Tome um emparelhamento para $G_{\mathcal{A}}$ e seja $\gamma: V\left(G_{\mathcal{A}}\right) \rightarrow \mathbb{Z}$ dada. Considere a realização $\mathcal{R}_{\mathcal{A}}$ do jogo $\mathcal{J}\left(G_{\mathcal{A}}, \gamma_{\mathcal{A}}\right)$ em que confrontam-se a estratégia de busca $\mathcal{E}_{\mathcal{A}}$ e a estratégia de evasão $\mathcal{E}$.

É imediato da definição da estratégia de evasão $\mathcal{E}$ e de $G_{\mathcal{A}}$ ser um emparelhamento que, para todo $v \in V\left(G_{\mathcal{A}}\right)$, o lance $\left(v, r_{\mathcal{A}, v}\right)$ é um lance de $\mathcal{R}_{\mathcal{A}}$. Seja $\delta_{\mathcal{A}}: V\left(G_{\mathcal{A}}\right) \rightarrow\{0,1\}$ a função dada por $\delta_{\mathcal{A}}(v)=r_{\mathcal{A}, v}$, onde $r_{\mathcal{A}, v}$ é a resposta da pergunta $v$ em $\mathcal{R}_{\mathcal{A}}$. Isso é o mesmo que dizer que $\mathcal{A}\left(G_{\mathcal{A}}, \delta_{\mathcal{A}}, \gamma\right)=V\left(G_{\mathcal{A}}\right)$.

A fim de poder provar um resultado similar ao Lema 1.1, vamos definir um outro jogo, que denotaremos por $\mathcal{F}$, e que modela instâncias $(G, \delta, \gamma)$ do $\mathrm{OD} k$ em que $G$ é um emparelhamento.

Principiamos por notar que dado um emparelhamento $k$-partido, $k$-uniforme $G$, cada vértice $v \in V(G)$ está completamente determinado por um par $(i, e)$ indicando a intersecção $V_{i}(G) \cap e$ para cada $1 \leq i \leq k$ e cada $e \in E(G)$. 
A partir desta observação, dado um emparelhamento $G$ como acima, podemos definir o grafo bipartido $B(G)$ dado por $V_{1}(B(G))=\{1, \ldots, k\}$, $V_{2}(B(G))=E(G)$ e $E(B(G))=\left\{\{i, e\}: V_{i}(G) \cap e \neq \emptyset\right\}$. Deste modo fica estabelecida uma bijeção natural entre $V(G)$ e $E(B(G))$ e, conseqüentemente, entre os subconjuntos de $V(G)$ e os subconjuntos de $E(B(G)$ ).

Uma conseqüência imediata do Lema 4 (página 62) é que, dada uma instância $\mathcal{I}=(G, \delta, \gamma)$ do $\mathrm{OD} k$, uma avaliação A de $\mathcal{I}$ está completamente descrita pelo conjunto $A \cap V^{0}(G)$, uma vez que

$$
\mathrm{A}=\left(\mathrm{A} \cap V^{0}(G)\right) \cup V\left[G-\left(\mathrm{A} \cap V^{0}(G)\right)\right] .
$$

No que segue, vamos definir um jogo de esconder sobre grafos bipartidos no qual o objetivo do procurador é determinar um conjunto de arestas com certas propriedades. A idéia é que, dada uma instância $\mathcal{I}=(G, \delta, \gamma)$ do $\mathrm{OD} k$ onde $G$ é um emparelhamento e $\gamma(v)=1$ para todo $v \in V(G)$, consideramos uma realização do jogo $\mathcal{F}(B(G))$. O conjunto de arestas a que chega o procurador ao final de uma realização do jogo $\mathcal{F}$, por sua vez, é interpretado como uma avaliação de $\mathcal{I}$, de acordo com a interpretação exposta acima. Passamos agora à definição formal do jogo $\mathcal{F}$.

Dados um grafo $G$ e um conjunto $F \subseteq E(G)$, vamos denotar por $G\{F\}=(V(G), F)$ o subgrafo de $G$ induzido por $F$, dado por $E(G\{F\})=F$ e $V(G\{F\})=\{v \in E(G): e \in F\}$.

Dados um grafo bipartido $B$ e uma função $f: V_{1}(B) \rightarrow \mathbb{N}$, dizemos que um conjunto $F \subseteq E(B)$ é um $f$-subfator esquerdo de $B$ se, para todo $v_{1} \in V_{1}(B)$ e todo $v_{2} \in V_{2}(B)$,

$$
\begin{aligned}
\operatorname{deg}_{B\{F\}}\left(v_{1}\right) & \leq f\left(v_{1}\right) \mathrm{e} \\
\operatorname{deg}_{B\{F\}}\left(v_{2}\right) & \leq 1 .
\end{aligned}
$$

Se vale a igualdade para cada $v \in V_{1}(B)$, dizemos que $F$ é um $f$-fator esquerdo de $B$.

Jogo: Subfator Esquerdo de Grafo Bipartido $(\mathcal{F}(B, f)$ )

Início: os jogadores recebem um grafo bipartido $B$ e uma função $f: V_{1}(B) \rightarrow \mathbb{N}$; a ocultadora escolhe um $f$-subfator esquerdo $F$ de $B$.

Lance: cada pergunta é uma aresta $e \in E(B)$; a resposta é sim ou não conforme $e \in F$ ou não.

Fim: o procurador encontra-se em condições de determinar $F$. 
O jogo $\mathcal{F}$ é o assunto do Capítulo 4 desta parte do trabalho. Ali, definimos uma certa estratégia de evasão $\mathcal{E}$, e a seu respeito provamos o Teorema 4.6 (página 97), cujo enunciado reproduzimos a seguir.

Em toda realização do jogo $\mathcal{F}(B, f)$ com a estratégia de evasão $\mathcal{E}$ em que $B$ tem um $f$-fator esquerdo, o procurador pergunta todas as arestas em $\partial(v)$ para algum vértice $v \in V_{1}(B)$ e determina um $f$-fator esquerdo de $B$.

A partir do Teorema 4.6 podemos provar o seguinte resultado.

Corolário 1.2. Todo algoritmo determinístico $\mathcal{A}$ para o $\mathrm{OD} k \mathrm{~d}$ admite uma instância $\mathcal{I}_{\mathcal{A}}=\left(G_{\mathcal{A}}, \delta_{\mathcal{A}}, \gamma_{\mathcal{A}}\right)$ tal que $\mathcal{A}\left(\mathcal{I}_{\mathcal{A}}\right)=V_{i}\left(G_{\mathcal{A}}\right)$ para algum $i \in\{1, \ldots, k\}$.

Demonstração. Seja $\mathcal{A}$ um algoritmo determinístico para o ODkd. Para um dado $t>0$, seja $G_{\mathcal{A}}$ um emparelhamento de $t k$ arestas e seja $f:\{1, \ldots, k\} \rightarrow \mathbb{N}$ a função dada por $f(i)=t$ para todo $i \in\{1, \ldots, k\}$.

Seja $\mathcal{E}_{\mathcal{A}}$ a estratégia de busca para o jogo $\mathcal{F}$ correspondente ao Algoritmo $\mathcal{A}$ e considere a realização $\mathcal{R}_{\mathcal{A}}$ do jogo $\mathcal{F}\left(B\left(G_{\mathcal{A}}\right), f\right)$ onde confrontamse a estratégia de busca $\mathcal{E}_{\mathcal{A}}$ e a estratégia de evasão $\mathcal{E}$.

Como $B\left(G_{\mathcal{A}}\right)$ tem um $f$-fator esquerdo, pelo Teorema 4.6 concluimos que para algum $i \in\{1, \ldots, k\}$ é verdade que $\left(v, r_{\mathcal{A}, v}\right)$ é um lance de $\mathcal{R}_{\mathcal{A}}$ para todo $v \in V_{i}(G)$.

Fazendo $\gamma_{\mathcal{A}}(v)=1$ para todo $v \in V\left(G_{\mathcal{A}}\right)$ e $\delta_{\mathcal{A}}(v)=r_{\mathcal{A}, v}$, onde $r_{\mathcal{A}, v}$ é a resposta da pergunta $v$ em $\mathcal{R}_{\mathcal{A}}$ e $\delta_{\mathcal{A}}(v)=1$ se $v$ não é perguntado em $\mathcal{R}_{\mathcal{A}}$, temos que $\mathcal{A}\left(G_{\mathcal{A}}, \delta_{\mathcal{A}}, \gamma_{\mathcal{A}}\right)=V_{i}\left(G_{\mathcal{A}}\right)$. 


\section{Capítulo 2}

\section{Ordenação Dinâmica $k$-partida Seqüencial}

Neste capítulo estudamos a variante seqüencial do ODk.

Na Seção 2.1 apresentamos cotas para a competitividade de algoritmos determinísticos e aleatorizados para o ODks. Mostramos que nenhum algoritmo determinístico para o ODks pode ter competitividade melhor que $k$, e que nenhum algoritmo aleatorizado para o OD $k$ s pode ter competitividade melhor que $(k+1) / 2$. Estas cotas aplicam-se qualquer que seja a complexidade do algoritmo em questão.

Na Seção 2.2 apresentamos um algoritmo determinístico polinomial otimamente competitivo para o ODks. Este algoritmo não precisa conhecer todo o hipergrafo de antemão a fim de resolver o problema, uma vez que avalia cada aresta à medida que é lida. Esta é uma qualidade conveniente para a aplicação em bases de dados que motiva este estudo.

O caso particular do OD $k$ onde $k=2$ é digno de interesse por si próprio e, como tal, recebeu alguma atenção na literatura. Na Seção 2.3 focamos nossa atenção sobre o OD2s. Apresentamos um algoritmo aleatorizado polinomial para o OD2s cuja competitividade é limitada por $2-\epsilon$ para todo $0 \leq \epsilon \leq 1-\sqrt{2} / 2$. A melhor competitividade deste algoritmo é $c=1+\sqrt{2} / 2$, atingida quando $\epsilon=1-\sqrt{2} / 2$. Entretanto, quanto maior o valor de $\epsilon$, menor é a probabilidade de uma particular execução deste algoritmo apresentar avaliação de competitividade $2-\epsilon$. Mostramos que a competitividade da avaliação computada por este algoritmo é limitada por $1+1 /(1-\epsilon)$. 
A competitividade deste algoritmo aleatorizado não tem garantia de concentrar-se em torno de seu valor esperado. Mostramos que esta limitação é inerente ao problema, e não uma desvantagem do enfoque apresentado. Mais precisamente, mostramos que para qualquer $0 \leq \epsilon \leq 1$, a avaliação computada por um algoritmo aleatorizado para o OD2s não pode ter competitividade melhor que $1+\epsilon$ com probabilidade maior do que $(1+\epsilon) / 2$.

\subsection{Cotas para Algoritmos Determinísticos e Ale- atorizados}

Nesta seção apresentamos cotas para a competitividade de algoritmos determinísticos e aleatorizados para o ODks, válidas qualquer que seja a complexidade do algoritmo em questão.

Corolário 2.1. Nenhum algoritmo determinístico para o ODks tem competitividade melhor que $k$.

Demonstração. Seja $(G, \delta, \gamma)$ uma instância do ODk onde $G$ é um emparelhamento e $\gamma(v)=1$ para todo $v$ in $V(G)$. Seja ainda $\mathcal{A}$ um algoritmo determinístico para o OD $k$ e considere a execução de $\mathcal{A}$ sobre a instância $\mathcal{I}_{\mathcal{A}}=\left(G, \delta_{\mathcal{A}}, \gamma\right)$.

Por definição, a competitividade de $\mathcal{A}$ é

$$
c(\mathcal{A}) \geq c\left(\mathcal{A}, \mathcal{I}_{\mathcal{A}}\right)=\frac{\gamma_{\mathrm{s}}\left(\mathcal{A}\left(\mathcal{I}_{\mathcal{A}}\right)\right)}{\gamma_{\mathrm{s}}^{*}\left(\mathcal{I}_{\mathcal{A}}\right)} .
$$

Pelo Lema 1.1 temos $\gamma_{\mathbf{s}}\left(\mathcal{A}\left(\mathcal{I}_{\mathcal{A}}\right)\right)=\gamma_{\mathbf{s}}(V(G))=|V(G)|$. Por outro lado, $V^{0}(G)$ é uma avaliação ótima para $\mathcal{I}_{\mathcal{A}}$ e, portanto,

$$
\gamma_{\mathbf{s}}^{*}\left(\mathcal{I}_{\mathcal{A}}\right)=\gamma\left(V^{0}(G)\right)=\left|V^{0}(G)\right|=|E(G)|
$$

Como $G$ é um emparelhamento, temos $|V(G)|=k|E(G)|$. Voltando a (2.1) temos

$$
c(\mathcal{A}) \geq \frac{\gamma_{\mathbf{s}}\left(\mathcal{A}\left(\mathcal{I}_{\mathcal{A}}\right)\right)}{\gamma_{\mathbf{s}}^{*}\left(\mathcal{I}_{\mathcal{A}}\right)}=\frac{|V(G)|}{|E(G)|}=\frac{k|E(G)|}{|E(G)|}=k
$$

Teorema 2.2. Nenhum algoritmo aleatorizado para o ODks tem competitividade melhor que $(k+1) / 2$. 
Demonstração. Considere o conjunto $\left\{I_{i}: 1 \leq i \leq k\right\}$ das instâncias $\left(G, \delta_{i}, \gamma\right)$, $1 \leq i \leq k$ do OD $k$ s nas quais $\gamma(v)=1$ para todo $v \in V(G), G$ tem um única aresta e, nessa aresta, um único vértice falso, que é o vértice $v \in e \cap V_{i}(G)$.

Seja $\mathcal{I}$ o espaço de probabilidades sobre o conjunto das instâncias do ODks onde as únicas instâncias com probabilidade positiva são as instâncias $I_{i}, 1 \leq i \leq k$, cada uma delas com a mesma probabilidade, a saber, $1 / k$. Aplicando o Teorema 2 (página 56) $\operatorname{com} \gamma_{\mathbf{s}}$ no papel da função $g$, temos

$$
\begin{aligned}
& \min \left\{\mathbb{E}_{\mathcal{I}}\left[\gamma_{\mathrm{s}}(\mathcal{A})\right]: \mathcal{A} \text { é um algoritmo determinístico para o ODks }\right\} \\
& \leq \max \left\{\mathbb{E}\left[\gamma_{\mathrm{s}}(\mathcal{R}(I))\right]: I \text { é uma instância do ODks }\right\}
\end{aligned}
$$

Seja $\mathcal{A}^{*}$ um algoritmo determinístico para o OD $k$ s satisfazendo

$$
\mathbb{E}_{\mathcal{I}}\left[\gamma_{\mathbf{s}}\left(\mathcal{A}^{*}\right)\right]=\min \left\{\mathbb{E}_{\mathcal{I}}\left[\gamma_{\mathbf{s}}(\mathcal{A})\right]: \mathcal{A} \text { é alg. determ. para ODks }\right\},
$$

e seja $\mathcal{E}_{\mathcal{A}^{*}}$ a estratégia de busca para o jogo $\mathcal{J}$ correspondente a $\mathcal{A}^{*}$. Para cada $1 \leq i \leq k$, a realização $R_{i}$ do jogo $\mathcal{J}(G, \gamma)$ com estratégia de busca $\mathcal{E}_{\mathcal{A}^{*}}$ é uma sequiência de perguntas que termina quando o vértice perguntado é o vértice falso.

Como $\mathcal{A}^{*}$ é um algoritmo determinístico, essa seqüência é sempre a mesma até o ponto em que termina. Podemos assumir sem perda de generalidade que $R_{i}=\left(\left(v_{1}, a_{1}\right), \ldots,\left(v_{i}, a_{i}\right)\right)$. Isto significa que $\gamma_{\mathrm{s}}\left(\mathcal{A}^{*}\left(I_{i}\right)\right)=i$ para cada $1 \leq i \leq k$. A partir de (2.3) temos

$\min \left\{\mathbb{E}_{\mathcal{I}}\left[\gamma_{\mathrm{s}}(\mathcal{A})\right]: \mathcal{A}\right.$ é um algoritmo determinístico para o ODks $\}$

$$
=\mathbb{E}_{\mathcal{I}}\left[\gamma_{\mathrm{s}}\left(\mathcal{A}^{*}\right)\right]=\sum_{i=1}^{k} \mathbb{P}_{\mathcal{I}}\left(I_{i}\right) \gamma_{\mathrm{s}}\left(\mathcal{A}^{*}\left(I_{i}\right)\right)=\sum_{i=1}^{k} \frac{1}{k} i=\frac{k+1}{2} .
$$

Seja $\mathcal{R}$ um algoritmo aleatorizado para o ODks. Combinando (2.2) e (2.4) temos

$\max \left\{\mathbb{E}\left[\gamma_{s}(\mathcal{R}(I))\right]: I\right.$ é uma instância do OD $\left.k \mathrm{~s}\right\}$

$\geq \min \left\{\mathbb{E}_{\mathcal{I}}\left[\gamma_{\mathrm{s}}(\mathcal{A})\right]: \mathcal{A}\right.$ é um algoritmo determinístico para o $\left.\mathrm{OD} k \mathrm{~s}\right\}$

$$
=\frac{k+1}{2} \text {. }
$$


Combinando a definição de competitividade de (6) com (2.5) temos

$$
\begin{aligned}
c(\mathcal{R})=\max \left\{\frac{\mathbb{E}\left[\gamma_{\mathrm{s}}(\mathcal{R}(I))\right]}{\gamma_{\mathbf{s}}^{*}(I)}: I \text { é uma instância do OD } k \mathrm{~s}\right\} \\
\geq \max \left\{\frac{\mathbb{E}\left[\gamma_{\mathbf{s}}\left(\mathcal{R}\left(I_{i}\right)\right)\right]}{\gamma_{\mathbf{s}}^{*}\left(I_{i}\right)}: 1 \leq i \leq k\right\} \\
\quad=\max \left\{\frac{\mathbb{E}\left[\gamma_{\mathbf{s}}\left(\mathcal{R}\left(I_{i}\right)\right)\right]}{1}: 1 \leq i \leq k\right\}=\frac{k+1}{2} .
\end{aligned}
$$

\subsection{Um Algoritmo Determinístico Otimamente Com- petitivo}

Nesta seção apresentamos $\mathcal{P}$, um algoritmo determinístico polinomial otimamente competitivo para o ODks, baseado no método de aproximação primal-dual (veja, por exemplo, Vazirani (2001)).

Dada uma instância $(G, \delta, \gamma)$ para o ODk, o Algoritmo $\mathcal{P}$ mantém uma 'variável dual' $y_{e}$, para cada $e \in E(G)$. Para cada vértice $v \in V(G)$, definimos a folga $f_{v}$ de $v$ como sendo a diferença entre o custo de $v$ e a soma dos valores das 'variáveis duais' $y_{e}$ das arestas $e \in E(G)$ que contém $v$, isto é

$$
f_{v}=\gamma(v)-\sum_{v \in e} y_{e}
$$

Após inicializar o valor de cada variável $y_{e}, e \in E(G)$ com 0 , e de cada $f_{v}, v \in V(G) \operatorname{com} \gamma(v)$, o Algoritmo $\mathcal{P}$ lê as arestas de $G$ uma a uma. Para cada aresta $e$ examinada, se o valor de $\bar{\delta}(e)$ ainda não pode ser determinado, o algoritmo avalia esta aresta através do Algoritmo $\mathcal{V}$.

Dada uma aresta $e \in E(G)$, o Algoritmo $\mathcal{V}(e)$ avalia os vértices de $e$ ainda não avaliados seguindo a ordem não decrescente de suas folgas até que um vértice falso seja avaliado ou até que o último vértice de $e$ seja avaliado. 


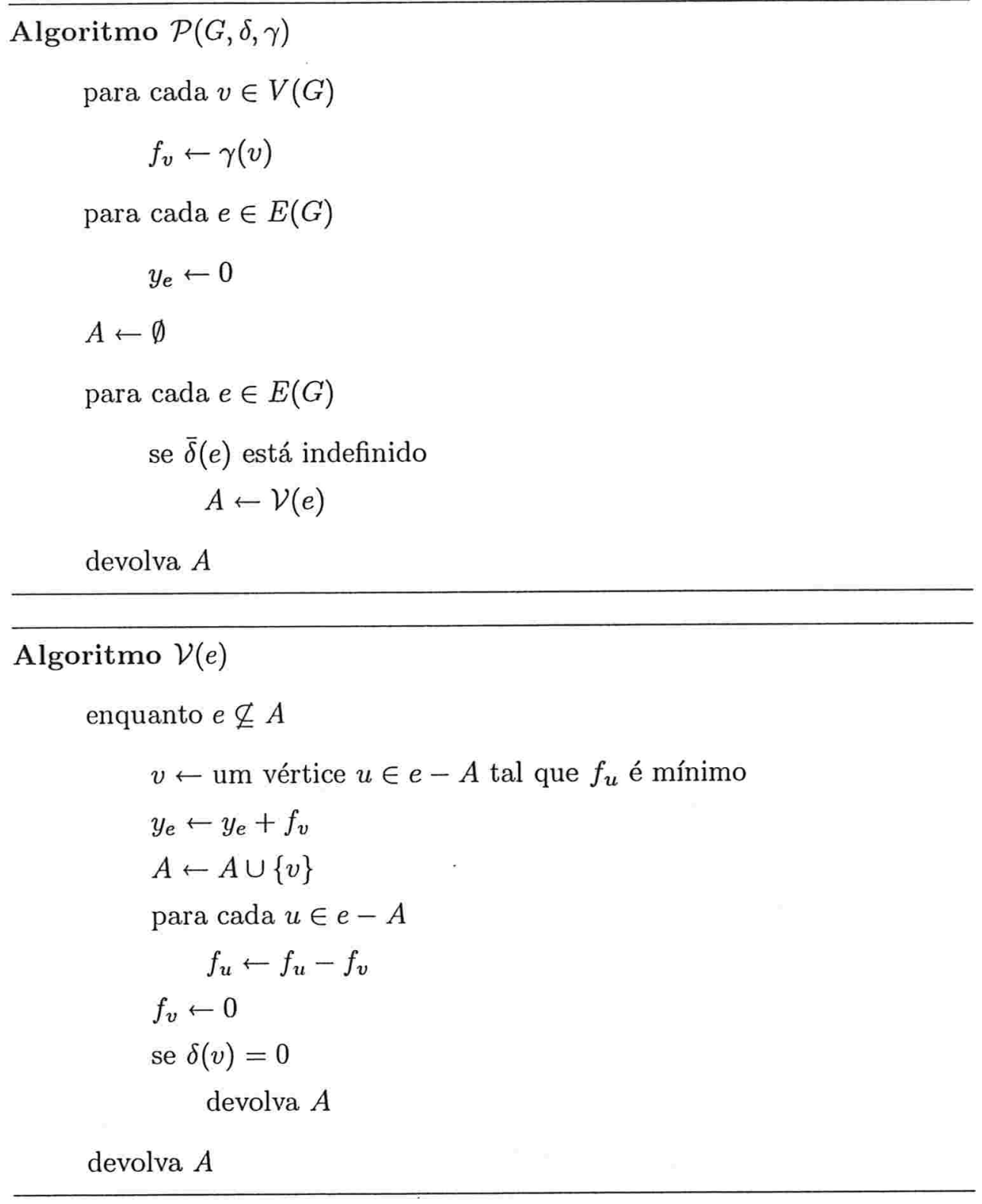

Teorema 2.3. O Algoritmo $\mathcal{P}$ é um algoritmo otimamente competitivo para $o$ OD $k$ s com complexidade $O(k|E(G)| \log |V(G)|)$.

Demonstração. Seja $\mathcal{I}=(G, \delta, \gamma)$ uma instância para o ODks, seja $\mathrm{A}^{*}$ uma 
avaliação ótima para $\mathcal{I}$ e seja

$$
J=\{e \in E(G): \mathcal{V}(e) \text { é executado }\} .
$$

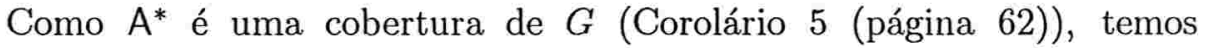
A $^{*} \cap e \neq \emptyset$ para todo $e \in J$. Para cada $e \in J$, seja $v^{*}$ o vértice de $\mathrm{A}^{*} \cap e$ com folga máxima imediatamente antes da execução de $\mathcal{V}(e)$. Temos que ou $v^{*}$ é o último vértice avaliado no laço de $\mathcal{V}$, ou então que $v^{*}$ não é avaliado. Em qualquer caso, temos $f_{v^{*}}=\max \left\{f_{u}: u \in e\right\}$ imediatamente após a execução de $\mathcal{V}(e)$ e podemos concluir que para todo $e \in J$,

$$
\sum_{v \in \mathrm{A}^{*} \cap e} f_{v} \geq \max \left\{f_{v}: v \in e\right\}
$$

Ao fim da execução de $\mathcal{P}(\mathcal{I})$, temos $\gamma(v) \geq \sum_{e \in J} y_{e}$, e então,

$$
\gamma\left(\mathrm{A}^{*}\right)=\sum_{v \in \mathrm{A}^{*}} \gamma(v) \geq \sum_{e \in J} \sum_{v \in \mathrm{A}^{*} \cap e} f_{v} \geq \sum_{e \in J} \max \left\{f_{v}: v \in e\right\}
$$

Por outro lado, se $v$ é avaliado então $\gamma(v)=\sum_{e \in J} y_{e}$, e segue que

$$
\gamma(\mathcal{P}(\mathcal{I}))=\sum_{v \in \mathcal{P}(\mathcal{I})} \sum_{e \in J} y_{e}=\sum_{e \in J} \sum_{v \in \mathcal{P}(\mathcal{I})} f_{v} \leq \sum_{e \in J}|e| \max \left\{f_{v}: v \in e\right\} .
$$

Da definição de competitividade em (5) combinada com (2.6) e (2.7), segue

$$
c(\mathcal{P}(\mathcal{I}), \mathcal{I})=\frac{\gamma(\mathcal{P}(\mathcal{I}))}{\gamma\left(\mathrm{A}^{*}\right)} \leq|e|=k,
$$

e daí mais do Corolário 2.1 segue que $c(\mathcal{P})=k$.

A etapa de inicialização do Algoritmo $\mathcal{P}$ toma tempo $O(|V(G)|+|E(G)|)$. A seguir, o Algoritmo $\mathcal{V}$ é executado no máximo $|E(G)|$ vezes. Para cada aresta $e, \mathcal{V}(e)$ monta uma fila de prioridades para os vértices ainda não avaliados da aresta $e-A$ e a seguir os extrai desta fila um por um, o que pode ser feito em tempo $O(k \log k)$ no pior caso. Por fim, o laço interno do Algoritmo $\mathcal{V}$ pode ser executado em tempo $O(k \log |V(G)|)$, mantendo-se uma árvore de busca equilibrada para $V(G)$ ao longo de toda a execução. A complexidade do Algoritmo $\mathcal{P}$ é, portanto, $O(k|E(G)| \log |V(G)|)$. 


\subsection{Ordenação Dinâmica 2-partida Seqüencial}

Nesta seção focamos nossa atenção sobre o caso particular do ODks em que $k=2$. Apresentamos um algoritmo aleatorizado polinomial para o OD2s cuja competitividade é limitada por $2-\epsilon$ para todo $0 \leq \epsilon \leq 1-\sqrt{2} / 2$. A melhor competitividade deste algoritmo é $c=1+\sqrt{2} / 2$, atingida quando $\epsilon=1-\sqrt{2} / 2$. Entretanto, quanto maior o valor de $\epsilon$, menor é a probabilidade de uma particular execução deste algoritmo apresentar avaliação de competitividade $2-\epsilon$. Mostramos que a competitividade de uma avaliação computada por este algoritmo é limitada por $1+1 /(1-\epsilon)$.

A competitividade deste algoritmo aleatorizado não tem garantia de concentrar-se em torno de seu valor esperado. Na Seção 2.3.1 mostramos que esta limitação é inerente ao problema, e não uma desvantagem do enfoque apresentado, provando que para qualquer $0 \leq \epsilon \leq 1$, a avaliação computada por um algoritmo aleatorizado para o OD2s não pode ter competitividade melhor que $1+\epsilon$ com probabilidade maior do que $(1+\epsilon) / 2$ para todas as instâncias do OD2s.

Lema 2.4. Dados uma instância $\mathcal{I}=(G, \delta, \gamma)$ do OD2s e uma cobertura $C$ de $G$, então uma $\mathrm{A}(C)=C \cup \Gamma^{1}(C)$ é uma avaliação de $\mathcal{I}$.

Demonstração. É imediato que $\mathrm{A}(C) \cap V^{1}(G) \supseteq V\left(G^{1}\right)$ e que $C^{0}=\mathrm{A}(C) \cap V^{0}(G)$ é uma cobertura de $E\left(G^{0}\right)$ restrita a $V^{0}(G)$. De acordo com o Lema 4 (página 62 ), $\mathrm{A}(C)$ é uma avaliação de $\mathcal{I}$.

Dada uma instância $\mathcal{I}=(G, \delta, \gamma)$ do OD2s, e uma cobertura $C$ de $G$, definimos uma $C$-avaliação de $\mathcal{I}$ como o conjunto $\mathrm{A}(C)=C \cup \Gamma^{1}(C)$.

Corolário 2.5. Seja $\mathcal{I}=(G, \delta, \gamma)$ uma instância do OD2s. Uma $C$-avaliação de $\mathcal{I}$ é uma avaliação otimamente competitiva de $\mathcal{I}$, se $C$ é uma cobertura minima de $\left(G, \gamma_{\mathrm{s}}\right)$.

O Corolário 2.5 sugere imediatamente o seguinte algoritmo para o OD2s.

$$
\begin{aligned}
& \text { Estratégia } \mathcal{C}(G, \delta, \gamma) \\
& \qquad \leftarrow \text { uma cobertura mínima de }(G, \gamma) \\
& \text { para cada } v \in C, \text { avalie } v \\
& \text { para cada } v \in \Gamma^{1}(C), \text { avalie } v
\end{aligned}
$$


Teorema 2.6. $O$ Algoritmo $\mathcal{C}$ é um algoritmo otimamente competitivo para o OD2s de complexidade $O\left(|V(G)|^{3}\right)$.

Demonstração. Pelo Corolário 2.5, o Algoritmo $\mathcal{C}$ é otimamente competitivo.

Dada uma instância $(G, \delta, \gamma)$ do OD2s, o problema de determinar uma cobertura mínima $C$ de $(G, \gamma)$ pode ser reduzido ao problema de determinar um corte mínimo num grafo dirigido de $|V(G)|+2$ vértices e $|E(G)|+|V(G)|$ arcos. Portanto, a cobertura $C$ pode ser computada em tempo $O\left(|V(G)|^{3}\right)$ (veja, por exemplo, Cook, Cunningham, Pulleyblank, and Schrijver (1997)).

O propósito desta seção é apresentar um algoritmo aleatorizado para o OD2s mais competitivo que o Algoritmo $\mathcal{C}$. Um exame da demonstração do Corolário 2.1 revela que uma classe de instâncias para as quais o Algoritmo $\mathcal{C}$ apresenta pior competitividade são instâncias onde a cobertura $C$ escolhida contém "muitos" vértices verdadeiros com vizinhos falsos. A idéia do algoritmo que passamos a apresentar consiste em verificar a possibilidade de ocorrência dessa configuração e, em caso positivo, escolher convenientemente outra cobertura em substituição de $C$.

De maneira um pouco mais precisa, seja $0 \leq \epsilon \leq 1$ dado e considere uma instância $\mathcal{I}$ do OD2s tal que $c(\mathcal{C}, \mathcal{I}) \geq 2-\epsilon$. Seja $C$ a cobertura mínima utilizada pelo Algoritmo $\mathcal{C}$, isto é $\mathcal{C}(\mathcal{I})=\mathrm{A}(C)$. A idéia é determinar uma outra cobertura $C_{\epsilon}$ de $(G, \gamma)$ cuja intersecção com $C$ é "pequena" e cujo custo "não é muito maior" que $\gamma(C)$.

Corolário 2.7. Toda avaliação de uma instância $(G, \delta, \gamma)$ do OD2s contém $\Gamma^{1}(V(G))$.

Demonstração. Por decorrência imediata do Lema 4 (página 62), e do fato de que $G$ é bipartido.

Lema 2.8. Seja $\mathcal{I}=(G, \delta, \gamma)$ uma instância do OD2s, seja $T \subseteq \Gamma^{1}(V(G))$, e seja $C_{T}$ uma cobertura mínima de $(G-T, \gamma)$. Então $\gamma\left(C_{T}\right)+\gamma(T) \leq \gamma^{*}(\mathcal{I})$.

Demonstração. Seja $A^{*}$ uma avaliação ótima para $\mathcal{I}$. De $T \subseteq \Gamma^{1}(V(G))$ segue pelo Corolário 2.7 que $T \subset A^{*}$. Além disso, $A^{*}-T$ tem que ser uma cobertura de $G-T$, pois senão $\bar{\delta}(u v)$ não poderia ser determinada para algum $u v \in E(G-T)$. Como $C_{T}$ é por escolha uma cobertura mínima de $G-T$, segue que $\gamma\left(C_{T}\right) \leq \gamma\left(A^{*}-T\right)$. Daí,

$$
\gamma\left(C_{T}\right)+\gamma(T) \leq \gamma\left(\mathrm{A}^{*}-T\right)+\gamma(T)=\gamma\left(\mathrm{A}^{*}\right)=\gamma^{*}(\mathcal{I}) .
$$


Lema 2.9. Seja $\mathcal{I}=(G, \delta, \gamma)$ uma instância do OD2s, seja $C$ uma cobertura minima de $(G, \gamma)$ e seja $0<\epsilon<1$. Se

$$
c(\mathrm{~A}(C), \mathcal{I}) \geq 2-\epsilon,
$$

então existe uma cobertura $C_{\epsilon}$ para $G$ tal que

$$
\gamma\left(C_{\epsilon}\right) \leq \frac{\gamma\left(C-C_{\epsilon}\right)}{1-\epsilon} .
$$

Demonstração. Seja $T=\Gamma^{1}(C)-C$. De (2.8) segue que

$$
2-\epsilon \leq \frac{\gamma(C)+\gamma(T)}{\gamma^{*}(\mathcal{I})} \text {. }
$$

Seja $C_{T}$ uma cobertura mínima de $G-T$. Da definição de $T$ segue que $T \subseteq \Gamma^{1}(V(G))$ e daí, pelo Lema 2.8 segue que $\gamma\left(C_{T}\right)+\gamma(T) \leq \gamma^{*}(\mathcal{I})$.

Um cálculo simples nos leva a

$$
\gamma(T) \leq \gamma(C)-(2-\epsilon) \frac{\gamma\left(C_{T}\right)}{1-\epsilon} .
$$

Tome $C_{\epsilon}=C_{T} \cup T$ e note que $C_{\epsilon}$ é uma cobertura de $G$ com $\left(C_{\epsilon} \cap C\right) \subset C_{T}$. Além disso,

$\gamma\left(C_{\epsilon}\right) \leq \gamma\left(C_{T}\right)+\gamma(T) \leq \frac{\gamma(C)-\gamma\left(C_{T}\right)}{1-\epsilon} \leq \frac{\gamma(C)-\gamma\left(C_{\epsilon} \cap C\right)}{1-\epsilon}=\frac{\gamma\left(C-C_{\epsilon}\right)}{1-\epsilon}$.

Seja $\mathcal{I}=(G, \delta, \gamma)$ e sejam $C$ e $\epsilon$ como no enunciado do Lema 2.9. Seja $C^{\prime}$ uma cobertura mínima de $\left(G, \gamma_{C, \epsilon}\right)$, onde $\gamma_{C, \epsilon}$ é dado por

$$
\gamma_{C, \epsilon}(v)= \begin{cases}(1-\epsilon) \gamma(v), & \text { se } v \notin C ; \\ (2-\epsilon) \gamma(v), & \text { caso contrário. }\end{cases}
$$

Formulando o problema de achar a cobertura $C_{\epsilon}$ que satisfaz (2.9) como um sistema linear ficamos com

$$
\begin{aligned}
\sum_{v \in V(G)} \gamma(v) x_{v} & \leq \frac{\sum_{v \in C} \gamma(v)\left(1-x_{v}\right)}{1-\epsilon} \\
x_{u}+x_{v} & \geq 1, \text { para todo } u v \in E(G),
\end{aligned}
$$


que tem solução em $\{0,1\}^{|V(G)|}$ se e somente se uma tal cobertura $C_{\epsilon}$ existe. Entretanto, através de uma simples manipulação algébrica percebe-se que sistema acima é equivalente a

$$
\begin{aligned}
\sum_{v \in C}(2-\epsilon) x_{v}+\sum_{v \in V(G)-C}(1-\epsilon) \gamma(v) x_{v} & \leq \gamma(C) \\
x_{u}+x_{v} & \geq 1, \text { para todo } u v \in E(G),
\end{aligned}
$$

e este sistema linear tem solução em $\{0,1\}^{|V(G)|}$ se e somente se $\gamma_{C, \epsilon}\left(C^{\prime}\right) \leq \gamma(C)$. Além disso se $\gamma_{C, \epsilon}\left(C^{\prime}\right) \leq \gamma(C)$ então

$$
\gamma\left(C^{\prime}\right) \leq \frac{\gamma\left(C-C^{\prime}\right)}{1-\epsilon}
$$

Este último comentário, junto com o Lema 2.9, nos dá uma maneira eficiente de verificar se $\mathrm{A}(C)$ é uma avaliação competitiva para $(G, \delta, \gamma)$ ou não, dada uma cobertura mínima $C$ de $(G, \gamma)$.

Como uma cobertura $C^{\prime}$ como acima pode ser computada em tempo polinomial, podemos escrever um algoritmo de tempo polinomial aleatorizado para OD2s que determina uma cobertura mínima $C$ de $(G, \gamma)$ e uma cobertura mínima $C^{\prime}$ de $\left(G, \gamma_{C, \epsilon}\right)$. Se $\gamma_{C, \epsilon}\left(C^{\prime}\right)>\gamma(C)$, então o Lema 2.9 assegura que $\mathrm{A}(C)$ será uma avaliação de competitividade melhor que $2-\epsilon$ para $(G, \delta, \gamma)$ e o algoritmo devolve $\mathrm{A}(C)$. Caso Contrário, o algoritmo devolve $\mathrm{A}(C)$ com probabilidade $p=p(\epsilon)$ ou $\mathrm{A}\left(C^{\prime}\right)$ com probabilidade $1-p$ como solução.

$$
\begin{aligned}
& \text { Algoritmo } \mathcal{R}_{\epsilon}(G, \delta, \gamma) \\
& C \leftarrow \text { uma cobertura mínima de }(G, \gamma) \\
& C^{\prime} \leftarrow \text { uma cobertura mínima de }\left(G, \gamma_{C, \epsilon}\right) \\
& \text { se } \gamma_{C, \epsilon}\left(C^{\prime}\right)>\gamma(C) \text {, devolva } \mathrm{A}(C) \\
& p \leftarrow \frac{1-3 \epsilon+\epsilon^{2}}{1-2 \epsilon} \\
& x \leftarrow \text { um número aleatório uniformemente escolhido em }[0,1] \\
& \text { se } x<p \text {, devolva } \mathrm{A}(C) \text {, caso contrário devolva } \mathrm{A}\left(C^{\prime}\right)
\end{aligned}
$$

Como $C$ é uma cobertura mínima de $(G, \gamma)$, temos que $\gamma(C) \leq \gamma\left(C^{\prime}\right)$. Soa razoável escolher $C$ com probabilidade maior do que $1 / 2$, isto é $p \geq 0.5$. Impondo esta condição sobre o valor de $p$ chegamos a $0 \leq \epsilon \leq 1-\sqrt{2} / 2$. 
A fim de estabelecer a competitividade do algoritmo $\mathcal{R}_{\epsilon}$, enunciamos o seguinte resultado auxiliar.

Lema 2.10. Sejam $C$ e $C^{\prime}$ duas coberturas de um grafo $G$. Toda aresta de $G$ com uma ponta fora de $C \cup C^{\prime}$ tem de ter sua outra ponta em $C \cap C^{\prime}$.

Demonstração. Sejam $v \in V(G)-\left(C \cup C^{\prime}\right)$ e $u \in \Gamma(v)$. Temos de ter $u \in C$, caso contrário $C$ não cobriria $u v$. Pelo mesmo argumento concluímos que $u \in C^{\prime}$ e, portanto, $u \in C \cap C^{\prime}$.

Teorema 2.11. Para todo $0 \leq \epsilon \leq 1-\sqrt{2} / 2$, o Algoritmo $\mathcal{R}_{\epsilon}$ é um algoritmo de competitividade $2-\epsilon$ e complexidade $O\left(|V(G)|^{3}\right)$ para o ODks.

Demonstração. Sejam $\mathcal{I}=(G, \delta, \gamma), C, C^{\prime}, \epsilon$ e $p$ como no enunciado do Algoritmo $\mathcal{R}_{\epsilon}$.

A correção do algoritmo $\mathcal{R}_{\epsilon}$ segue pelo Lema 2.4 do fato de que $C$ e $C^{\prime}$ são coberturas de $G$. Também é imediato que sua complexidade é dominada pela complexidade do cálculo das coberturas de custo mínimo do grafo $G$, e, portanto, é $O\left(|V(G)|^{3}\right.$ ) (veja a prova do Teorema 2.6).

Para calcular a competitividade do Algoritmo $\mathcal{R}_{\epsilon}$, começamos por definir os seguintes conjuntos (veja Figura 2.1 para uma representação esquemática).

$$
\begin{aligned}
H & =\Gamma^{1}\left(C \cap C^{\prime}\right)-\left(C \cup C^{\prime}\right), \\
H_{C} & =\Gamma^{1}\left(C^{\prime}\right) \cap\left(C-C^{\prime}\right), \\
H_{C^{\prime}} & =\Gamma^{1}(C) \cap\left(C^{\prime}-C\right) .
\end{aligned}
$$

Do Lema 2.10 segue

$$
\begin{aligned}
H \cup H_{C^{\prime}} & =\Gamma^{1}(C)-C=\Gamma^{1}(C)-\left(C \cap \Gamma^{1}(C)\right), \\
H \cup H_{C} & =\Gamma^{1}\left(C^{\prime}\right)-C^{\prime}=\Gamma^{1}\left(C^{\prime}\right)-\left(C^{\prime} \cap \Gamma^{1}\left(C^{\prime}\right)\right),
\end{aligned}
$$

e, como $H, H_{C}, H_{C^{\prime}} \subseteq \Gamma^{1}(V(G))$ são conjuntos disjuntos, temos

$$
\begin{aligned}
\gamma(\mathrm{A}(C)) & =\gamma(C)+\gamma\left(H_{C^{\prime}}\right)+\gamma(H), \\
\gamma\left(\mathrm{A}\left(C^{\prime}\right)\right) & =\gamma\left(C^{\prime}\right)+\gamma\left(H_{C}\right)+\gamma(H), \\
\gamma^{*}(\mathcal{I}) & \geq \gamma(H)+\gamma\left(H_{C}\right)+\gamma\left(H_{C^{\prime}}\right) .
\end{aligned}
$$

Queremos mostrar que $c\left(\mathcal{R}_{\epsilon}(\mathcal{I}), \mathcal{I}\right) \leq 2-\epsilon$. Partindo da definição (6) temos

$$
c\left(\mathcal{R}_{\epsilon}(\mathcal{I}), \mathcal{I}\right)=\frac{\mathbb{E}\left[\gamma\left(\mathcal{R}_{\epsilon}(\mathcal{I})\right)\right]}{\gamma^{*}(\mathcal{I})} .
$$




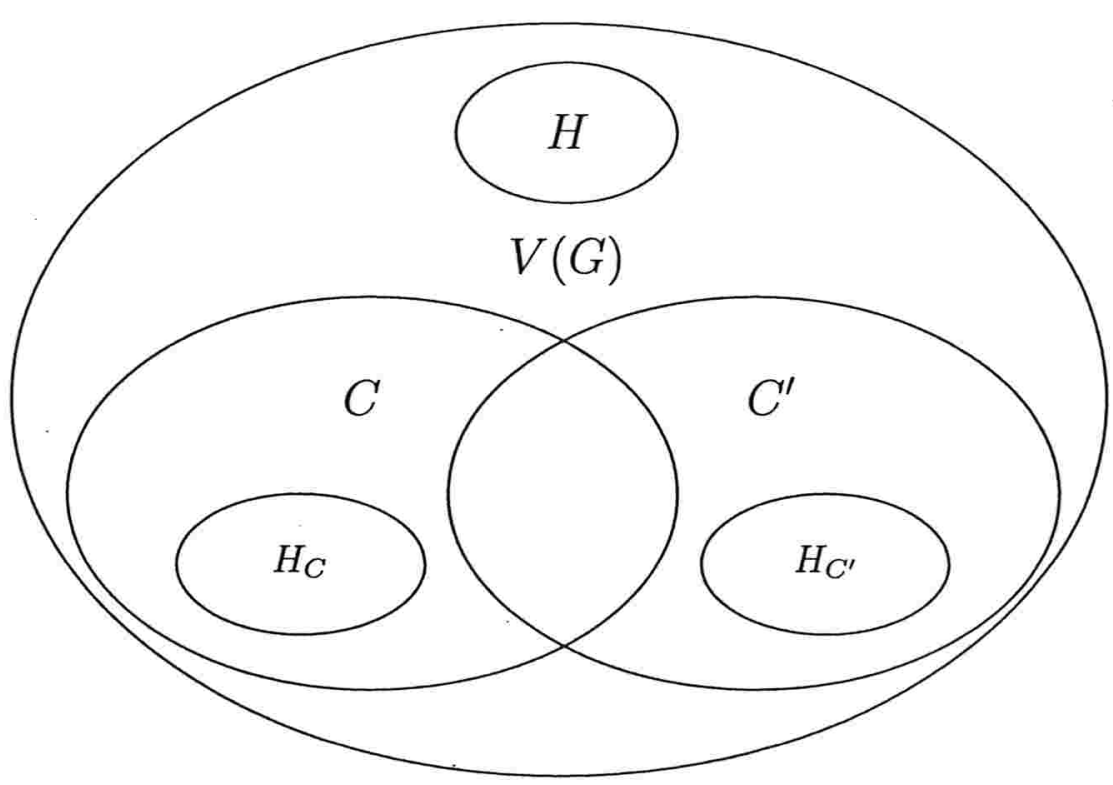

Figura 2.1: Os conjuntos $C, C^{\prime}, H_{C}, H_{C^{\prime}}$ e $H$

Do enunciado do Algoritmo $\mathcal{R}_{\epsilon}$ combinado a (2.11) e (2.12) temos

$$
\begin{aligned}
& \mathbb{E}\left[\gamma\left(\mathcal{R}_{\epsilon}(\mathcal{I})\right)\right]=p \gamma(\mathrm{A}(C))+(1-p) \gamma\left(\mathrm{A}\left(C^{\prime}\right)\right) \\
& \quad=p \gamma(C)+(1-p) \gamma\left(C^{\prime}\right)+(1-p) \gamma\left(H_{C}\right)+p \gamma\left(H_{C^{\prime}}\right)+\gamma(H)
\end{aligned}
$$

Seja $C_{H}$ uma cobertura mínima de $(G-H, \gamma)$. Como cada aresta de $G-H$ é coberta por $C_{H}$ e toda aresta incidente a $H$ é coberta por um vértice de $C \cap C^{\prime}$, podemos aplicar o Lema 2.10 a $G[H]$ e concluir que $C_{H} \cup\left(C \cap C^{\prime}\right)$ é uma cobertura de $G$. Como $C$ é uma cobertura mínima de $G$, temos que $\gamma(C) \leq \gamma\left(C_{H} \cup\left(C \cap C^{\prime}\right)\right) \leq \gamma\left(C_{H}\right)+\gamma\left(C \cap C^{\prime}\right)$, ou, equivalentemente, $\gamma\left(C_{H}\right) \geq \gamma(C)-\gamma\left(C \cap C^{\prime}\right) \geq \gamma\left(C-C^{\prime}\right)$.

Como $H \subseteq \Gamma^{1}(V(G))$, segue do Lema 2.8 que

$$
\gamma^{*}(\mathcal{I}) \geq \gamma(H)+\gamma\left(C-C^{\prime}\right)
$$

de maneira que, do fato de que $C$ é uma cobertura mínima e das desigualdades (2.13) e (2.16), temos,

$$
\gamma^{*}(\mathcal{I}) \geq \max \left\{\gamma(C), \gamma(H)+\gamma\left(H_{C}\right)+\gamma\left(H_{C^{\prime}}\right), \gamma(H)+\gamma\left(C-C^{\prime}\right)\right\}
$$


Como a escolha de $\epsilon$ implica $p \geq 0.5$, segue de (2.15) e (2.17) que

$$
\mathbb{E}\left[\gamma_{\mathbf{s}}\left(\mathcal{R}_{\epsilon}(\mathcal{I}), \mathcal{I}\right)\right] \leq 2 p+(1-p) \frac{\gamma\left(C^{\prime}\right)+\gamma(H)}{\gamma(H)+\gamma\left(C-C^{\prime}\right)} .
$$

Para $p=\left(1-3 \epsilon+\epsilon^{2}\right) /(1-2 \epsilon)$ e lembrando a desigualdade (2.10) temos $c\left(\mathcal{R}_{\epsilon}\right) \leq 2-\epsilon$.

Teorema 2.12. Para toda instância $\mathcal{I}$ do OD2s temos que

$$
c\left(\mathcal{R}_{\epsilon}(\mathcal{I}), \mathcal{I}\right) \leq 1+\frac{1}{1-\epsilon} \text {. }
$$

Demonstração. Primeiro, note que se $\mathcal{R}_{\epsilon}(\mathcal{I})=\mathrm{A}(C)$, então $c\left(\mathcal{R}_{\epsilon}(\mathcal{I}), \mathcal{I}\right) \leq 2$, pois $C$ é uma cobertura mínima. Por outro lado, se $\mathcal{R}_{\epsilon}(\mathcal{I})=\mathrm{A}\left(C^{\prime}\right)$, então

$$
c\left(\mathcal{R}_{\epsilon}(\mathcal{I}), \mathcal{I}\right) \leq \frac{\gamma\left(C^{\prime}\right)+\gamma\left(H_{C}\right)+\gamma(H)}{\max \left\{\gamma(C), \gamma\left(H_{C}\right)+\gamma(H)\right\}},
$$

e então, lembrando a desigualdade (2.10) temos

$$
c\left(\mathcal{R}_{\epsilon}(\mathcal{I}), \mathcal{I}\right) \leq \frac{2-\epsilon}{1-\epsilon}=\frac{1}{1-\epsilon} .
$$

O seguinte resultado mostra que a cota de $2-\epsilon$ para a competitividade de $\mathcal{R}_{\epsilon}$ é a melhor possível.

Teorema 2.13. Seja $\epsilon=1-\sqrt{2} / 2$. Existe uma familia de instâncias $\mathcal{I}_{\alpha}$ do ODks satisfazendo

$$
\lim _{\alpha \rightarrow \sqrt{2}} c\left(\mathcal{R}_{\epsilon}\left(\mathcal{I}_{\alpha}\right), \mathcal{I}_{\alpha}\right)=2-\epsilon
$$

Demonstração. Dado $\alpha>1$, considere as instâncias $\mathcal{I}_{\alpha}=(G, \delta, \gamma)$, em que $G$ é um grafo bipartido completo com $\left|V_{2}(G)\right|=\alpha\left|V_{1}(G)\right|, \delta(v)=0$ para todo $v \in V_{1}(G), \delta(v)=1$ para todo $v \in V_{2}(G)$, e $\gamma(v)=1$ para todo $v \in V(G)$.

Claramente, $V_{1}(G)$ é uma cobertura mínima de $(G, \gamma)$ e $V_{2}(G)$ é uma cobertura mínima de $\left(G, \gamma_{C, \epsilon}\right)$ com $\gamma_{C, \epsilon}\left(V_{2}(G)\right) \leq \gamma\left(V_{1}(G)\right)$, de forma que $\mathcal{R}_{\epsilon}\left(\mathcal{I}_{\alpha}\right)$ avalia $\mathrm{A}\left(V_{1}(G)\right)=V_{1}(G)$ com probabilidade $1 / 2$ e avalia $\mathrm{A}\left(V_{2}(G)\right)=V(G)$ também com probabilidade $1 / 2$. Temos então que $d\left(\mathcal{R}_{\epsilon}\left(\mathcal{I}_{\alpha}\right), \mathcal{I}_{\alpha}\right)=1+\alpha / 2$, e daí

$$
\lim _{\alpha \rightarrow \sqrt{2}} c\left(\mathcal{R}_{\epsilon}\left(\mathcal{I}_{\alpha}\right), \mathcal{I}_{\alpha}\right)=\lim _{\alpha \rightarrow \sqrt{2}} 1+\frac{\alpha}{2}=1+\frac{\sqrt{2}}{2}=2-\epsilon .
$$




\subsubsection{Cota para a Probabilidade da Competitividade}

Se por um lado, o Teorema 2.11 mostra que $2-\epsilon$ é uma cota para a competitividade do Algoritmo $\mathcal{R}_{\epsilon}$, por outro lado a demonstração do Teorema 2.13 evidencia que a probabilidade de a avaliação $\mathcal{R}_{\epsilon}(\mathcal{I})$ atingir tal competitividade não é alta, isto é, não se concentra em torno do valor de $c\left(\mathcal{R}_{\epsilon}(\mathcal{I}), \mathcal{I}\right)$.

Nesta seção mostramos que essa não é uma peculiaridade do Algoritmo $\mathcal{R}_{\epsilon}$, mas uma característica presente em qualquer algoritmo aleatorizado para o OD2s. Mais precisamente, mostramos no Corolário 2.18 (página 86) que nenhum algoritmo aleatorizado para o OD2s pode calcular para todas as instâncias do OD2s uma avaliação de competitividade melhor que $\mu$ com probabilidade maior que $\mu / 2$, qualquer que seja o valor de $1 \leq \mu \leq 2$.

Corolário 2.14. Seja $l \geq 0$, seja $\mathcal{I}$ um espaço de probabilidades de suporte finito cujos eventos elementares são as instâncias do OD2s. Para todo algoritmo aleatorizado $\mathcal{R}$ para o OD2s vale que

$$
\begin{gathered}
\min \left\{\mathbb{P}_{\mathcal{I}}\left(\gamma_{\mathrm{s}}(\mathcal{A}(\mathcal{I})) \geq l\right): \mathcal{A} \text { é um algoritmo determinístico para o OD2s }\right\} \\
\leq \max \left\{\mathbb{P}\left(\gamma_{\mathrm{s}}(\mathcal{R}(\mathcal{I})) \geq l\right): \mathcal{I} \text { é uma instância do OD2s }\right\} .
\end{gathered}
$$

Demonstração. Por aplicação direta do Corolário 3 (página 56) tomando para $P$ o predicado $P(A)=\mathbb{P}(\mathrm{A} \geq l)$.

A seguir, vamos definir uma distribuição de probabilidades conveniente para as instâncias do OD2s e aplicar o Corolário 2.14 para provar nosso resultado.

Para cada $n>0$ par e cada $1 \leq i \leq n$, seja $I_{i, n}=\left(G_{n}, \delta_{i, n}, \gamma_{n}\right)$ a instância do OD2s em que $G_{n}$ é um grafo bipartido completo com $V_{1}(G)=\{1, \ldots, n / 2\}$ e $V_{2}(G)=\{n / 2+1, \ldots, n\}, \gamma_{n}(v)=1$ para todo $v \in V\left(G_{n}\right)$ e $\delta_{i, n}$ dada por

$$
\delta_{i, n}(v)= \begin{cases}1, & \text { se } i=v \\ 0, & \text { caso contrário. }\end{cases}
$$

Lema 2.15. O custo de uma avaliação ótima da instância $I_{i, n}$ é

$$
\gamma_{\mathbf{s}}^{*}\left(I_{i, n}\right)=n / 2
$$

para todo $n>0$ e todo $1 \leq i \leq n$. 
Demonstração. De acordo com o Corolário 2.7, toda avaliação de $I_{i, n}$ tem que conter $\Gamma^{1}\left(V\left(G_{n}\right)\right)$. Da definição de $I_{i, n}$ temos que

$$
\Gamma^{1}\left(V\left(G_{n}\right)\right) \in\left\{V_{1}(G), V_{2}(G)\right\} .
$$

Logo, toda avaliação $\mathrm{A}$ de $\mathcal{I}_{i, n}$ tem custo pelo menos

$$
\gamma_{\mathrm{s}}(\mathrm{A}) \geq \min \left\{\left|V_{1}(G)\right|,\left|V_{2}(G)\right|\right\}=n / 2 .
$$

Por outro lado, temos que $V_{2}(G)$ é uma avaliação de custo $n / 2$ de $I_{i, n}$ para todo $1 \leq i \leq n / 2$ e que $V_{1}(G)$ é uma avaliação de custo $n / 2$ de $I_{i, n}$ para todo $n / 2+1 \leq i \leq n$. Assim, $I_{i, n}$ tem uma avaliação de custo $n / 2$ para todo $1 \leq i \leq n$.

Lema 2.16. Dados $n>0$ par e $0 \leq t \leq 1 / 2$, todo algoritmo determinístico para o OD2s computa uma avaliaçao de custo pelo menos $n / 2+$ tn para pelo menos $n / 2-t n+1$ das instâncias $I_{i, n}, 1 \leq i \leq n$.

Demonstração. Seja $\mathcal{A}$ um algoritmo determinístico para o OD2s e considere a estratégia de busca $\mathcal{E}_{\mathcal{A}}$ correspondente a $\mathcal{A}$. Seja $R=\left(\left(v_{1}, 0\right), \ldots,\left(v_{k}, 0\right)\right)$ a realização do jogo $\mathcal{J}\left(G_{n}, \gamma_{n}\right)$ obtida ao confrontar a Estratégia $\mathcal{E}_{\mathcal{A}}$ com a estratégia de evasão que responde 0 em todos os lances do jogo. Nesse caso, $k \geq n / 2$ e $v_{k}$ é o "último vértice" de uma das classes de vértices de $G_{n}$, isto é, $\left\{v_{1}, \ldots, v_{k-1}\right\} \notin\left\{V_{1}\left(G_{n}\right), V_{2}\left(G_{n}\right)\right\}$ e $\left\{v_{1}, \ldots, v_{k}\right\} \in\left\{V_{1}\left(G_{n}\right), V_{2}\left(G_{n}\right)\right\}$. Sem perda de generalidade, assumamos que $V_{1}\left(G_{n}\right) \subseteq\left\{v_{1}, \ldots, v_{k}\right\}$.

Daí é imediato que a execução de $\mathcal{A}\left(I_{v_{k}, n}\right)$ avalia todos os vértices de $V\left(G_{n}\right)$, isto é, $\mathcal{A}\left(I_{v_{k}, n}\right)=V\left(G_{n}\right)$. Em geral, para cada $v \in\left\{v_{1}, \ldots, v_{k}\right\}$ vale que $\mathcal{A}\left(I_{v, n}\right)=V_{2}\left(G_{n}\right) \cup\left(\left\{v_{1}, \ldots, v_{j}=v\right\} \cap V_{1}\left(G_{n}\right)\right)$. Assim, para pelo menos $n / 2-$ tn instâncias $I_{i, n}, 1 \leq i \leq n$ temos $\gamma_{\mathbf{s}}\left(\mathcal{A}\left(I_{i, n}\right)\right) \geq n / 2+t n$.

Para cada $n>0$ par, seja $\mathcal{I}_{n}$ a distribuição de probabilidades sobre as instâncias do ODks onde as únicas instâncias com probabilidade positiva são as $n$ instâncias $I_{i, n}, 1 \leq i \leq n$, todas elas equiprováveis.

Lema 2.17. Para todo $n>0$ par e todo $1 / 2 \leq \lambda \leq 1$,

$$
\min \left\{\mathbb{P}_{\mathcal{I}_{n}}\left(\gamma_{\mathrm{s}}(\mathcal{A}(I)) \geq \lambda n\right): \mathcal{A} \text { é alg. determin. para o ODks }\right\}>1-\lambda .
$$

Demonstração. Seja $\mathcal{A}$ um algoritmo determinístico para o OD2s. Fazendo $t=\lambda-1 / 2$, segue do Lema 2.16 que

$$
\left|\left\{\mathcal{I}_{i, n}: 1 \leq i \leq n, \gamma_{\mathbf{s}}\left(\mathcal{A}\left(\mathcal{I}_{i, n}\right)\right) \geq n / 2+t n\right\}\right| \geq n / 2-t n+1,
$$


e, portanto,

$$
\begin{aligned}
& \mathbb{P}_{\mathcal{I}_{n}}\left(\gamma_{\mathrm{s}}(\mathcal{A}(\mathcal{I}))\right.\geq \lambda n) \\
&=\frac{1}{n} \mid\left\{\mathcal{I}_{i, n}: 1 \leq i \leq n, \gamma_{\mathrm{s}}\left(\mathcal{A}\left(\mathcal{I}_{i, n}\right)\right)\right.\geq(t+1 / 2) n\} \mid \\
& \geq \frac{1}{n}\left(\frac{n}{2}-t n+1\right)=\frac{1}{2}-t+\frac{1}{n}=\frac{1}{2}-\left(\lambda-\frac{1}{2}\right)+\frac{1}{n}=1-\lambda+\frac{1}{n} \\
&>1-\lambda .
\end{aligned}
$$

Corolário 2.18. Nenhum algoritmo aleatorizado para o OD2s computa uma avaliação de competitividade melhor que $\mu$ com probabilidade maior que $\mu / 2$ para todas as instâncias do OD2s.

Demonstração. Seja $\mathcal{R}$ um algoritmo aleatorizado para o OD2s. Combinando (2.19) e (2.21) temos

$$
\max \left\{\mathbb{P}\left(\gamma_{\mathbf{s}}(\mathcal{R}(I)) \geq \lambda n\right): I \text { é uma instância do OD2s }\right\}>1-\lambda .
$$

Em particular, para todo $n>0$, par, temos

$$
\mathbb{P}\left(\gamma_{s}\left(\mathcal{R}\left(I_{i, n}\right)\right) \geq \lambda n\right)>1-\lambda .
$$

para algum $1 \leq i \leq n$. Combinando (2.24), com (2.20) e a definição de competitividade em (4) temos

$$
\begin{aligned}
1-\lambda< & \mathbb{P}\left(\gamma_{\mathbf{s}}\left(\mathcal{R}\left(I_{i, n}\right)\right) \geq \lambda n\right)=\mathbb{P}\left(\frac{\gamma_{\mathrm{s}}\left(\mathcal{R}\left(I_{i, n}\right)\right)}{\gamma_{\mathbf{s}}^{*}\left(\mathcal{I}_{i, n}\right)} \geq \frac{\lambda n}{\gamma_{\mathbf{s}}^{*}\left(I_{i, n}\right)}\right) \\
= & \mathbb{P}\left(c\left(\mathcal{R}\left(I_{i, n}\right), I_{i, n}\right) \geq \frac{\lambda n}{n / 2}\right)=\mathbb{P}\left(c\left(\mathcal{R}\left(I_{i, n}\right), I_{i, n}\right) \geq 2 \lambda\right) .
\end{aligned}
$$

Fazendo $\mu=2 \lambda$ em (2.25) concluimos que

$$
\mathbb{P}\left(c\left(\mathcal{R}\left(\mathcal{I}_{i, n}\right), \mathcal{I}_{i, n}\right)<\mu\right) \leq \frac{\mu}{2} .
$$




\section{Capítulo 3}

\section{Ordenação Dinâmica $k$-partida Distribuída}

Neste capítulo estudamos a variante distribuída do OD $k$. Na Seção 3.1 apresentamos cotas para a competitividade de algoritmos determinísticos para o ODkd. e na Seção 3.2 apresentamos algoritmos determinísticos para o ODkd. Apresentamos um algoritmo polinomial otimamente competitivo para o OD $k$ d segundo o modelo preemptivo e um algoritmo exponencial otimamente competitivo para o ODkd segundo o modelo não-preemptivo.

\subsection{Cotas para a Competitividade de Algoritmos Determinísticos}

Nesta seção apresentamos cotas para a competitividade de algoritmos determinísticos para o ODkd. Mostramos que nenhum algoritmo determinístico para o OD $k$ d pode ter competitividade melhor que $k$ qualquer que seja a complexidade do algoritmo em questão.

O seguinte resultado estabelece formalmente uma cota inferior natural para o custo da solução ótima de uma instância do ODkd em termos do custo da solução ótima da mesma instância para o ODks.

Lema 3.1. Para toda instância I do ODkd temos

$$
\gamma_{\mathrm{d}}^{*}(\mathcal{I}) \geq \frac{\gamma_{\mathrm{s}}^{*}(\mathcal{I})}{k} .
$$

Demonstração. Seja $\mathcal{I}=(G, \delta, \gamma)$ e seja $\mathrm{A}^{*}$ uma avaliação ótima para $\mathcal{I}$, 
como instância do OD $k$ d. Claramente temos $\sum_{i=1}^{k} \gamma_{\mathrm{s}}\left(\mathrm{A}^{*} \cap V_{i}(G)\right) \geq \gamma_{\mathrm{s}}^{*}(\mathcal{I})$, e daí

$$
\gamma_{\mathbf{d}}^{*}(\mathcal{I}) \geq \max \left\{\gamma_{\mathbf{s}}\left(\mathrm{A}^{*} \cap V_{i}(G)\right): 1 \leq i \leq k\right\} \geq \frac{1}{k} \sum_{i=1}^{k} \gamma_{i}\left(\mathrm{~A}^{*}\right) \geq \frac{\gamma_{\mathrm{s}}^{*}(\mathcal{I})}{k}
$$

Corolário 3.2. Nenhum algoritmo determinístico para o $\mathrm{ODk \textrm {d }}$, quer no modelo preemptivo quer no modelo não-preemptivo, tem competitividade melhor que $k$.

Demonstração. Seja $\mathcal{A}$ um algoritmo determinístico para o ODkd e sejam $t$, $f$ e $\mathcal{I}_{\mathcal{A}}=\left(G_{\mathcal{A}}, \delta_{\mathcal{A}}, \gamma_{\mathcal{A}}\right)$ como na demonstração do Corolário 1.2.

Da construção de $G_{\mathcal{A}}$ e $\delta_{\mathcal{A}}$ temos que $G_{\mathcal{A}}$ tem $t k^{2}$ vértices, sendo $t k$ deles falsos, $t$ deles em cada classe de $V\left(G_{\mathcal{A}}\right)$. Daí temos que $V^{0}(G)$ é uma avaliação ótima de $\mathcal{I}_{\mathcal{A}}$ com custo $\gamma_{\mathrm{d}}\left(\mathcal{I}_{\mathcal{A}}\right)=\gamma_{\mathrm{d}}\left(V^{0}(G)\right)=t$, pois os $t$ vértices falsos de cada classe podem ser todos avaliados em paralelo.

De acordo com o Corolário 1.2 , temos $V_{i}(G) \subseteq \mathcal{A}\left(\mathcal{I}_{\mathcal{A}}\right)$ para algum $i \in\{1, \ldots, k\}$ e daí temos

$$
c(\mathcal{A}) \geq c\left(\mathcal{A}\left(\mathcal{I}_{\mathcal{A}}\right), \mathcal{I}_{\mathcal{A}}\right) \geq \frac{\gamma_{\mathrm{d}}\left(\mathcal{A}\left(\mathcal{I}_{\mathcal{A}}\right)\right)}{\gamma_{\mathrm{d}}^{*}\left(\mathcal{I}_{\mathcal{A}}\right)} \geq \frac{\left|V_{i}\left(G_{\mathcal{A}}\right)\right|}{t}=\frac{t k}{t}=k .
$$

\subsection{Algoritmos Determinísticos}

Nesta seção apresentamos algoritmos determinísticos para o ODkd. $\mathrm{Na}$ Seção 3.2.1 apresentamos uma versão modificada do Algoritmo $\mathcal{P}$ (da Seção 2.2) que resulta num algoritmo determinístico polinomial otimamente competitivo para o ODkd, no modelo preemptivo. Na Seção 3.2.2, apresentamos um algoritmo exponencial determinístico otimamente competitivo para o modelo não-preemptivo do ODkd.

\subsubsection{Modelo Preemptivo}

Seja $\mathcal{V}_{\mathbf{d}}$ a seguinte variante do Algoritmo $\mathcal{V}$, da Seção 2.2 . 


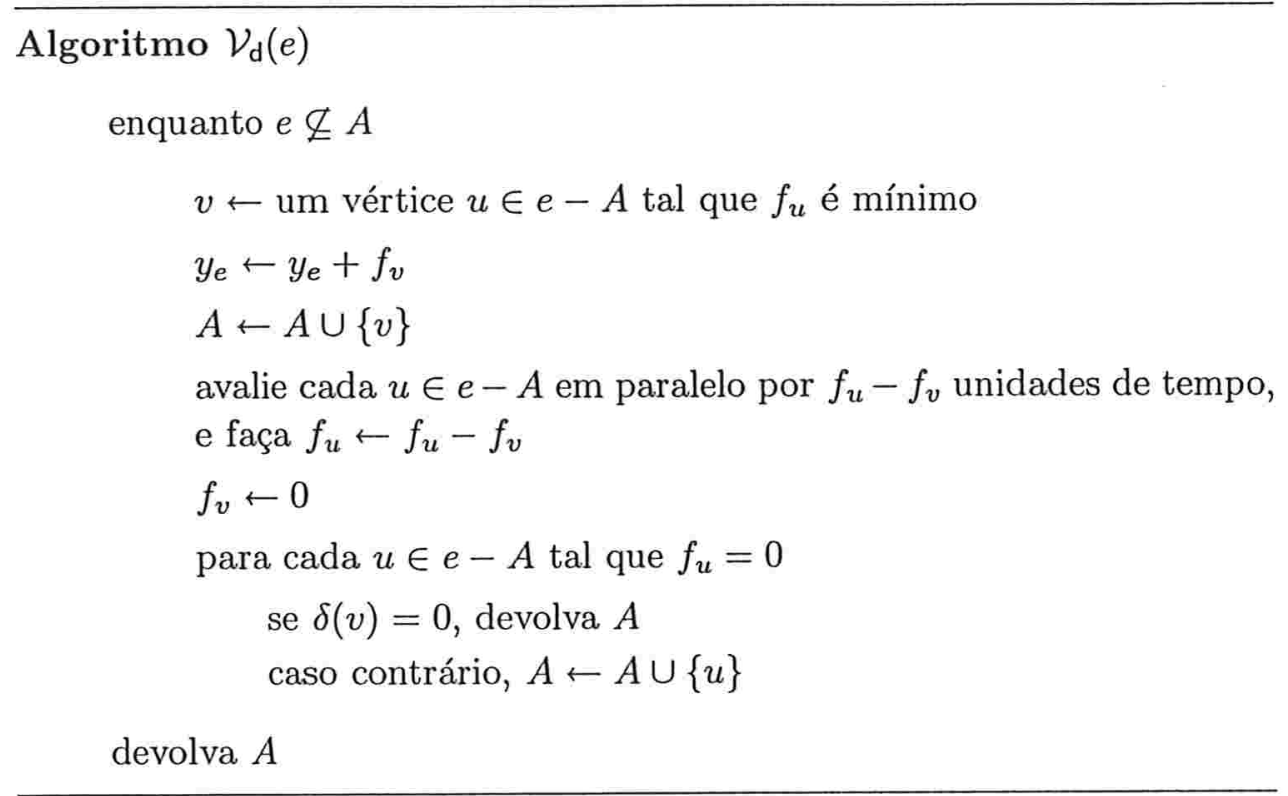

Seja agora $\mathcal{P}_{\mathrm{d}}$ o seguinte algoritmo para o OD $k$ d, resultante de trocar a execução do Algoritmo $\mathcal{V}$ pela execução do Algoritmo $\mathcal{V}_{\mathrm{d}}$.

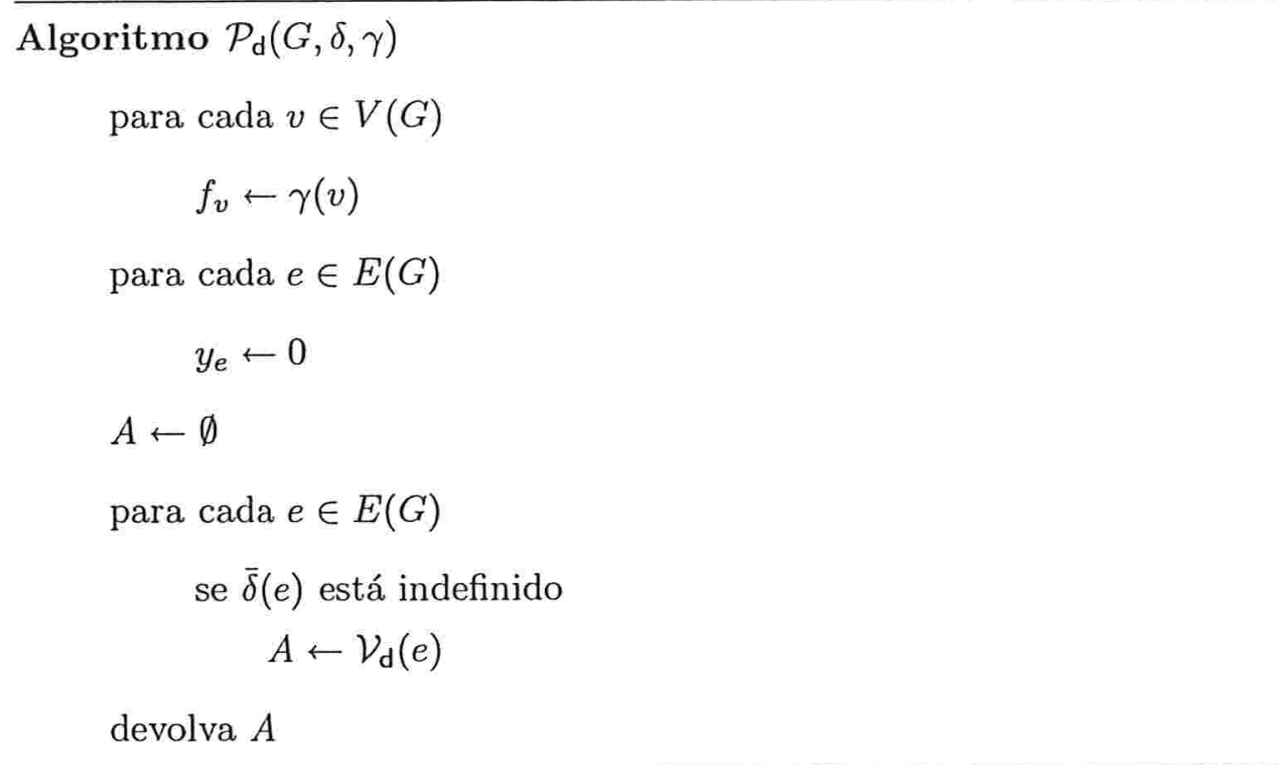

Teorema 3.3. Algoritmo $\mathcal{P}_{\mathrm{d}}$ é um algoritmo otimamente competitivo para $o \mathrm{ODkd}$ no modelo preemptivo. 
Demonstração. Seja $\mathcal{I}=(G, \delta, \gamma)$ uma instância do ODkd e, para cada $1 \leq i \leq k$, seja $\gamma_{i}\left(\mathcal{P}_{\mathrm{d}}(\mathcal{I})\right)$ o custo incorrido pelo processador a cargo de $V_{i}(G)$ na avaliação $\mathcal{P}_{\mathrm{d}}(\mathcal{I})$. De (2.6) temos

$$
\gamma_{\mathrm{d}}\left(\mathcal{P}_{\mathrm{d}}(\mathcal{I})\right) \geq \max \left\{\gamma_{i}\left(\mathcal{P}_{\mathrm{d}}(\mathcal{I})\right): 1 \leq i \leq k\right\} \leq \sum_{e \in J} \max \left\{f_{v}: v \in e\right\} \leq \gamma_{\mathrm{s}}^{*}(\mathcal{I})
$$

onde $J=\left\{e \in E(G): \mathcal{V}_{\mathrm{d}}(e)\right.$ é executado $\}$. Daí combinando com (3.1) temos

$$
c\left(\mathcal{P}_{\mathrm{d}}(\mathcal{I}), \mathcal{I}\right)=\frac{\gamma_{\mathrm{d}}\left(\mathcal{P}_{\mathrm{d}}(\mathcal{I})\right)}{\gamma_{\mathrm{d}}^{*}(\mathcal{I})} \leq \frac{\gamma_{\mathrm{s}}^{*}(\mathcal{I})}{\gamma_{\mathrm{s}}^{*}(\mathcal{I}) / k}=k
$$

\subsubsection{Modelo Não-Preemptivo}

Seja $\mathcal{I}=(G, \delta, \gamma)$ uma instância do ODkd, e seja $X \subseteq V(G)$. Definimos o custo distribuído de $X$ por

$$
\gamma_{\max }(X)=\max \left\{\gamma_{\mathrm{s}}\left(V_{i}(G) \cap X\right): 1 \leq i \leq k\right\} .
$$

Considere o seguinte algoritmo.

$$
\begin{aligned}
& \text { Algoritmo } \mathcal{D}(G, \delta, \gamma) \\
& \text { se } r(G)=1 \\
& \quad \text { avalie todos } v \in V(G) \text { em paralelo } \\
& C \leftarrow \text { uma cobertura de custo distribuído mínimo de }(G, \gamma) \\
& \text { avalie todo } v \in C \text { em paralelo } \\
& \text { para cada } v \in C \\
& \text { se } \delta(v)=0 \\
& \quad G \leftarrow G-v \\
& \text { caso contrário } \\
& \text { retire } v \text { de cada aresta de } G \\
& \mathcal{I}^{\prime} \leftarrow\left(G^{\prime}, \gamma^{\prime}, \delta^{\prime}\right) \leftarrow\left(G,\left.\delta\right|_{V\left(G^{\prime}\right)},\left.\gamma\right|_{V\left(G^{\prime}\right)}\right) \\
& \text { execute } \mathcal{D}\left(\mathcal{I}^{\prime}\right)
\end{aligned}
$$


Teorema 3.4. O Algoritmo $\mathcal{D}$ é um algoritmo otimamente competitivo para ODkd.

Demonstração. Mostraremos por indução sobre $k$ que $c(\mathcal{D}(\mathcal{I}), \mathcal{I}) \leq k$ para toda instância $\mathcal{I}$ do ODkd, e daí

$$
c(\mathcal{D})=\max \{c(\mathcal{D}(\mathcal{I}), \mathcal{I}): \mathcal{I} \text { é uma instância para o ODkd }\} \leq k \text {. }
$$

A base, $k=1$, é imediata pois a avaliação todos vértices de $G$ em paralelo sem tempo ocioso é claramente a avaliação ótima para qualquer instância do dD10.

Para o passo de indução, seja $k>1$ e assuma que o enunciado vale para todo $t<k$. Seja $\mathcal{I}=(G, \delta, \gamma)$ uma instância para o ODkd e seja $\mathrm{A}^{*}$ uma avaliação ótima para $\mathcal{I}$. Temos então

$$
c(\mathcal{D}(\mathcal{I}), \mathcal{I})=\frac{\gamma_{\mathrm{d}}(\mathcal{D}(\mathcal{I}))}{\gamma_{\mathrm{d}}\left(\mathrm{A}^{*}\right)} \leq \frac{\gamma_{\max }(C)+\gamma_{\mathrm{d}}\left(\mathcal{D}\left(\mathcal{I}^{\prime}\right)\right)}{\gamma_{\mathrm{d}}\left(\mathrm{A}^{*}\right)} .
$$

Note que, como $C$ é uma cobertura de $G$, temos $r\left(G^{\prime}\right)<r(G)=k$. Com a hipótese de indução temos

$$
\gamma_{\mathrm{d}}\left(\mathcal{D}\left(\mathcal{I}^{\prime}\right)\right) \leq(k-1) \gamma_{\mathrm{d}}^{*}\left(\mathcal{I}^{\prime}\right) .
$$

Claramente a avaliação $A^{*}$ restrita aos vértices de $G^{\prime}$ é uma avaliação para $\mathcal{I}^{\prime}$. Então, $\gamma_{\mathrm{d}}^{*}\left(\mathcal{I}^{\prime}\right) \leq \gamma_{\mathrm{d}}\left(\mathrm{A}^{*}\right)$, e daí

$$
\begin{aligned}
c(\mathcal{D}(\mathcal{I}), \mathcal{I}) \leq \frac{\gamma_{\max }(C)+\gamma_{\mathrm{d}}\left(\mathcal{D}\left(\mathcal{I}^{\prime}\right)\right)}{\gamma_{\mathrm{d}}\left(\mathrm{A}^{*}\right)} \leq & \frac{\gamma_{\max }(C)}{\gamma_{\mathrm{d}}\left(\mathrm{A}^{*}\right)}+\frac{(k-1) \gamma_{\mathrm{d}}^{*}\left(\mathcal{I}^{\prime}\right)}{\gamma_{\mathrm{d}}\left(\mathrm{A}^{*}\right)} \\
& \leq 1+(k-1) \frac{\gamma_{\mathrm{d}}^{*}\left(\mathcal{I}^{\prime}\right)}{\gamma_{\mathrm{d}}\left(\mathrm{A}^{*}\right)} \leq k
\end{aligned}
$$




\section{Capítulo 4}

\section{Um Jogo de Esconder Fatores de Grafo}

Neste capítulo, estudamos o jogo $\mathcal{F}$, apresentado na Seção 1. Para maior conveniência, repetimos aqui algumas das definições já apresentadas na Seção 1.

Dados um grafo bipartido $B$ e uma função $f: V_{1}(B) \rightarrow \mathbb{N}$, dizemos que um conjunto $F \subseteq E(B)$ é um $f$-subfator esquerdo de $B$ se, para todo $v_{1} \in V_{1}(B)$ e todo $v_{2} \in V_{2}(B)$,

$$
\begin{aligned}
\operatorname{deg}_{B\{F\}}\left(v_{1}\right) \leq f\left(v_{1}\right) \mathrm{e} \\
\operatorname{deg}_{B\{F\}}\left(v_{2}\right) \leq 1
\end{aligned}
$$

Se vale a igualdade para cada $v \in V_{1}(B)$, dizemos que $F$ é um $f$-fator esquerdo de $B$.

Por concisão, fixamos de uma vez por todas a seguinte notação e terminologia: $B$ denota um grafo bipartido e referimo-nos às classes de vértices $V_{1}(B)$ e $V_{2}(B)$ de $B$ como classes esquerda e direita. Elementos genéricos de cada classe são denotados por $l$ e $r$. Também, $f$ denota uma função $f: V_{1}(B) \rightarrow \mathbb{N}$.

Dado um conjunto $X \subseteq V_{1}(B)$, denotamos a restrição de $f$ a $X$ por $f_{X}$. Como usual, denotamos $f(X)=\sum_{x \in X} f(x)$. A restrição de $f$ a $V_{1}(B)-X$ é denotada $\overline{f_{X}}$. O subgrafo de $B$ induzido por $X \cup \Gamma_{B}(X)$ é denotado $B_{X}$ e seu complemento, $B-V\left(B_{X}\right)$ é denotado $\overline{B_{X}}$, isto é

$$
\begin{aligned}
& B_{X}=B\left[X \cup \Gamma_{B}(X)\right], \\
& \overline{B_{X}}=B-V\left(B_{X}\right)=B-\left(X \cup \Gamma_{B}(X)\right) .
\end{aligned}
$$


por

Para todo $l \in V_{1}(B) \operatorname{com} f(l)>0$, definimos a função $(f-l): V_{1}(B) \rightarrow \mathbb{N}$

$$
(f-l)(v)= \begin{cases}f(v)-1, & \text { se } v=l \\ f(v), & \text { caso contrário. }\end{cases}
$$

Jogo: Subfator Esquerdo de Grafo Bipartido $(\mathcal{F}(B, f))$

Início: os jogadores recebem um grafo bipartido $B$ e uma função $f: V_{1}(B) \rightarrow \mathbb{N}$; a ocultadora escolhe $F$, um $f$-subfator esquerdo de $B$.

Lance: cada pergunta é uma aresta $e \in E(B)$; a resposta é sim ou não conforme $e \in F$ ou não.

Fim: o procurador encontra-se em condições de determinar $F$.

\subsection{Alguns Resultados sobre Fatores Esquerdos}

Principiamos por formular um conhecido resultado (veja Bollobás (1978), Capítulo 2) a respeito de fatores de um grafo, numa forma conveniente para nossos propósitos.

Teorema 4.1. $O$ grafo $B$ tem um $f$-fator esquerdo se e somente se para todo $X \subseteq V_{1}(B)$, temos

$$
f(X) \leq\left|\Gamma_{B}(X)\right| \text {. }
$$

Corolário 4.2. Seja $e=\{l, r\} \in E(B)$ tal que $B$ tem um $f$-fator esquerdo $e$ $B-e$ não tem $f$-fator esquerdo. Então, e pertence a todo $f$-fator esquerdo de $B$ e existe um conjunto $l \in A \subseteq V_{1}(B)$ tal que

$$
f(A)=\left|\Gamma_{B}(A)\right| \text {. }
$$

Demonstração. Se $B-e$ não tem $f$-fator esquerdo, do Teorema 4.1 sabemos que existe algum $A \subseteq V_{1}(B-e)$ para o qual $f(A)>\left|\Gamma_{B-e}(A)\right|$. Por outro lado, como $B$ tem um $f$-fator esquerdo, temos $f(A) \leq\left|\Gamma_{B}(A)\right|$. Como $\left|\Gamma_{B-e}(A)\right| \leq\left|\Gamma_{B}(A)\right|+1$, concluímos que $f(A)=\left|\Gamma_{B}(A)\right|$ e $l \in A$.

O seguinte será enunciado sem prova, uma vez que é imediato das definições. 
Lema 4.3. Seja $F \subseteq E(B)$ e $e=\{l, r\} \in F$. $O$ conjunto $F$ é um $f$-fator esquerdo de $B$, se e somente se $F-\{e\}$ é um $(f-l)$-fator esquerdo de $B-r$.

O seguinte resultado dá informação sobre a estrutura de um $f$-fator esquerdo no caso em que vale a igualdade em (4.3) para um dado conjunto $A$. A Figura 4.1 mostra um exemplo de um grafo $B$ e um conjunto $A$ como no Lema 4.4. Neste exemplo, temos $f(l)=2$ para todo $l \in V_{1}(B)$. As arestas de $B_{A}$ e $\overline{B_{A}}$ são mostradas linhas reforçadas.

B

$L(B) \quad R(B)$

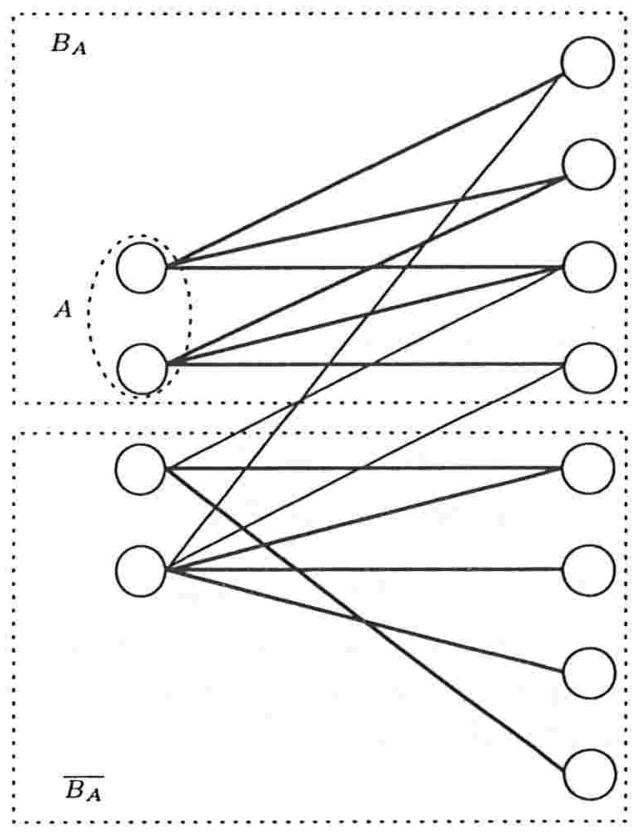

Figura 4.1: a decomposição em Lema 4.4

Lema 4.4. Seja $A \subseteq V_{1}(B)$ tal que $f(A)=\left|\Gamma_{B}(A)\right|$. Um conjunto $F \subseteq E(B)$ é um $f$-fator esquerdo de $B$ se e somente se $F=F_{A} \cup \overline{F_{A}}$, onde $F_{A}=F \cap E\left(B_{A}\right)$ é um $f_{A}$-fator esquerdo de $B_{A}$ e $\overline{F_{A}}=F \cap E\left(\overline{B_{A}}\right)$ é um $\overline{f_{A}}$-fator esquerdo de $\overline{B_{A}}$.

Demonstração. É imediato das definições que se $F_{A}$ é um $f_{A}$-fator esquerdo de $B_{A}$ e $\overline{F_{A}}$ é um $\overline{f_{A}}$-fator esquerdo de $\overline{B_{A}}$, então $F=F_{A} \cup \overline{F_{A}}$ é um $f$-fator esquerdo de $B$. 
Para provar a volta, basta mostrar que toda aresta em $F$ está em $E\left(B_{A}\right)$ ou em $E\left(\overline{B_{A}}\right)$.

Seja $e=\{l, r\} \in F-E\left(B_{A}\right)$. É imediato da definição de $B_{A}$ que $l \in V_{1}\left(\overline{B_{A}}\right)=V_{1}(B)-A$. Se $e \notin E\left(\overline{B_{A}}\right)$ então $r \notin V_{2}\left(\overline{B_{A}}\right)=V_{2}(B)-\Gamma_{B}(A)$, isto é, $r \in \Gamma_{B}(A)$. Isto não é possível, porque $e \in F$ e $\left|\Gamma_{B}(A)\right|=f(A)$.

\subsection{Estratégia de Evasão}

Dada uma realização $\mathcal{R}=\left(\left(e_{1}, a_{1}\right), \ldots,\left(e_{m}, a_{m}\right)\right)$ do jogo $\mathcal{F}(B, f)$, denotamos por $Q(\mathcal{R}, k)$ o conjunto de arestas consultadas pelo procurador até (e inclusive) a jogada $k$, e por $N(\mathcal{R}, k)$ o subconjunto dessas arestas para as quais a ocultadora respondeu não. Também, denotamos por $B(\mathcal{R}, k)$, o grafo obtido a partir de de $B$ ao apagar $N(\mathcal{R}, k)$, isto é

$$
\begin{aligned}
Q(\mathcal{R}, k) & =\left\{e_{i}: 1 \leq i \leq k\right\} \\
N(\mathcal{R}, k) & =\left\{e_{i}: 1 \leq i \leq k, a_{i}=\text { não }\right\} \\
B(\mathcal{R}, k) & =B-N(\mathcal{R}, k)
\end{aligned}
$$

Por conveniência, também fazemos

$$
\begin{aligned}
Q(\mathcal{R}) & =Q(\mathcal{R}, m), \\
N(\mathcal{R}) & =N(\mathcal{R}, m), \\
B(\mathcal{R}) & =B(\mathcal{R}, m) .
\end{aligned}
$$

No caso em que $B$ tem um $f$-fator esquerdo, definimos a Estratégia $\mathcal{S}$ para a ocultadora como a estratégia na qual ela sempre responde não a qualquer consulta, a menos que seja obrigada a responder sim a fim de ter um $f$-fator esquerdo no fim do jogo, isto é,

Estratégia $\mathcal{S}(B, f)$

$N \leftarrow \emptyset$

para cada pergunta $e$

se $B-(N \cup\{e\})$ não tem um $f$-fator, responda sim

caso contrário

$N \leftarrow N \cup\{e\}$

responda não 
Os seguintes comentários sublinham alguns outros fatos imediatos a respeito de realizações do jogo $\mathcal{F}(B, f)$ com a estratégia de evasão $\mathcal{S}$. Estes fatos serão importantes na prova do nosso resultado principal.

Observação 4.5. Em qualquer realização $\mathcal{R}=\left(\left(e_{1}, a_{1}\right), \ldots,\left(e_{m}, a_{m}\right)\right)$ do jogo $\mathcal{F}(B, f)$ em que a ocultadora segue a Estratégia $\mathcal{S}$ :

1. para todo $k \in\{1, \ldots, m\}$ vale que

$a_{k}=\operatorname{sim}$ se e somente se $B(\mathcal{R}, k-1)-e_{k}$ não tem $f$-fator esquerdo.

2. o conjunto de arestas de $B$ ao qual a ocultadora responde sim, a saber, $F=Q(\mathcal{R})-N(\mathcal{R})$, é um f-fator esquerdo de $B$. Além disso, $F$ é o único $f$-fator esquerdo de $B(\mathcal{R})$.

3. $A$ ocultadora responde sim a exatamente $f\left(V_{1}(B)\right)$ jogadas do jogo, isto é, $|Q(\mathcal{R})-N(\mathcal{R})|=f\left(V_{1}(B)\right)$.

4. Se $f\left(V_{1}(B)\right)>0$, o jogo termina precisamente na última jogada na qual a ocultadora responde sim, isto é, $a_{m}=\operatorname{sim}$.

Teorema 4.6. Em toda realização $\mathcal{R}$ do jogo $\mathcal{F}(B, f)$ na qual $B$ tem um $f$-fator esquerdo, $f\left(V_{1}(B)\right)>0$ e a estratégia de evasão $\mathcal{S}$ é usada,

$$
\partial_{B}(l) \subseteq Q(\mathcal{R}),
$$

para algum vértice $l \in V_{1}(B)$.

Demonstração. A prova é por indução sobre $f\left(V_{1}(B)\right)$.

Suponha $f\left(V_{1}(B)\right)=1$, e seja $\mathcal{R}=\left(\left(e_{1}, a_{1}\right), \ldots,\left(e_{m}, a_{m}\right)\right)$ uma realização de $\mathcal{F}(B, f)$. Do Comentário 4.5 .4 temos $a_{m}=\operatorname{sim}$. Seja $l$ a ponta de $e_{m}$ em $V_{1}(B)$. É claro que se houvesse uma aresta $e \in \partial_{B(\mathcal{R}, m)}(l)-\left\{e_{m}\right\}$, então $\{e\}$ seria um $f$-fator esquerdo de $B(\mathcal{R}, m-1)-e_{m}$, o que contradiz a Estratégia $\mathcal{S}$. Como conseqüência,

$$
\partial_{B}(l) \subseteq Q(\mathcal{R}, m)=Q(\mathcal{R}) .
$$

Consideremos agora o caso em que $f\left(V_{1}(B)\right)=t>1$ sob a presunção de que o enunciado vale quando quer que $f\left(V_{1}(B)\right)<t$.

Como antes, seja $\mathcal{R}=\left(\left(e_{1}, a_{1}\right), \ldots,\left(e_{m}, a_{m}\right)\right)$ e $k$ a primeira jogada na qual a ocultadora responde sim, isto é,

$$
k=\min \left\{1 \leq i \leq m: a_{i}=\operatorname{sim}\right\} .
$$


Para todo $1 \leq i \leq m$, seja $e_{i}=\left\{l_{i}, r_{i}\right\}$, com $l_{i} \in V_{1}(B)$ e $r_{i} \in V_{2}(B)$.

De acordo com a definição da Estratégia $\mathcal{S}$, o grafo $B(\mathcal{R}, k-1)-e_{k}$ tem um $f$-fator esquerdo, e então, pelo Corolário 4.2, sabemos que existe algum $A \subseteq V_{1}(B(\mathcal{R}, k-1))=V_{1}(B(\mathcal{R}, k))$ para o qual $f(A)=\left|\Gamma_{B(\mathcal{R}, k)}(A)\right|$.

Seja $F=Q(\mathcal{R})-N(\mathcal{R})$ o $f$-fator esquerdo de $B$ determinado pelo procurador em $\mathcal{R}$ (veja Comentário 4.5.2). Do Corolário 4.2 temos $e_{k} \in F \mathrm{e}$ $l_{k} \in A$.

Suponha que $f(A)=\left|\Gamma_{B(\mathcal{R}, k)}(A)\right|=1$. Então temos $\Gamma_{B(\mathcal{R}, k)}(A)=\left\{r_{k}\right\}$. É claro, então, que

$$
\partial_{B}\left(l_{k}\right) \subseteq\left\{e_{k}\right\} \cup N(\mathcal{R}, k) \subseteq Q(\mathcal{R}, k) \subseteq Q(\mathcal{R}) .
$$

Suponhamos agora que $f(A)=\left|\Gamma_{B(\mathcal{R}, k)}(A)\right|>1$ e seja $\mathcal{R}_{k}$ a subseqüência obtida de $\mathcal{R}$ apagando as jogadas $\left(e_{i}, a_{i}\right)$ em que $i \leq k$ ou $e_{i} \notin E\left(B_{A}\right)$ e sejam ainda

$$
\begin{aligned}
B_{k} & =B_{A}-r_{k}, \\
f_{k} & =f_{A}-l_{k} .
\end{aligned}
$$

Fato 4.7. A seqüência $\mathcal{R}_{k}$ é uma realização do jogo $\mathcal{F}\left(B_{k}, f_{k}\right)$ na qual a ocultadora segue a Estratégia $\mathcal{S}$.

Demonstração. Primeiro precisamos verificar que $\mathcal{F}\left(B_{k}, f_{k}\right)$ é um jogo bem definido, que a Estratégia $\mathcal{S}$ é elegível para a ocultadora neste jogo, e que $\mathcal{R}_{k}$ é uma realização dele.

Que $\mathcal{F}\left(B_{k}, f_{k}\right)$ é um jogo bem definido segue diretamente das definições de $B_{k}$ e $f_{k}$ em (4.7) e (4.8). Que $\mathcal{R}_{k}$ é uma realização deste jogo, segue da definição de $\mathcal{R}_{k}$. Finalmente, o fato de que a Estratégia $\mathcal{S}$ é elegível para a ocultadora neste jogo segue, primeiro da hipótese, onde $f\left(V_{1}(B)\right)>1$ implica $f_{k}\left(V_{1}\left(B_{k}\right)\right)>0$ e, segundo, do fato que $B_{k}$ tem um $f_{k}$-fator esquerdo $\mathcal{F}\left(B_{k}, f_{k}\right)$. Isto é assim porque, do Lema 4.4 , temos que $F \cap E\left(B_{A}\right)$ é um $f_{A}$-fator esquerdo de $B_{A}$ e daí, do Lema 4.3 , temos que $F-e_{k}$ é uma $f_{k}$-fator esquerda de $B_{k}=B_{A}-r_{k}$.

Seja $\mathcal{R}_{k}=\left(\left(e_{1}^{\prime}, a_{1}^{\prime}\right), \ldots,\left(e_{s}^{\prime}, a_{s}^{\prime}\right)\right)=\left(\left(e_{i_{1}}, a_{i_{1}}\right), \ldots,\left(e_{i_{s}}, a_{i_{s}}\right)\right)$ e seja $1 \leq j \leq s$. Da definição de $\mathcal{R}_{k}$ temos

$$
a_{j}^{\prime}=\operatorname{sim} \text { se e somente se } a_{i_{j}}=\operatorname{sim} .
$$

da definição da Estratégia $\mathcal{S}$ temos

$$
a_{i_{j}}=\text { sim se e somente se } B\left(\mathcal{R}, i_{j}-1\right)-e_{i_{j}} \text { tem um } f \text {-fator esquerdo. }
$$


Por conveniência, seja $B^{\prime}=B\left(\mathcal{R}, i_{j}-1\right)-e_{i_{j}}$ de maneira que (4.10) pode ser reescrita como

$$
a_{i_{j}}=\operatorname{sim} \text { se e somente se } B^{\prime} \text { não tem } f_{A} \text {-fator esquerdo. }
$$

É uma conseqüência do Lema 4.4 que

$B^{\prime}$ não tem $f_{A}$-fator esquerdo se e somente se $B_{A}^{\prime}$ não tem $f_{A}$-fator esquerdo.

Das definições em (4.1) e (4.5), temos

$$
\begin{aligned}
B_{A}^{\prime}=B^{\prime}\left[A \cup \Gamma_{B^{\prime}}(A)\right]=\left(B\left(\mathcal{R}, i_{j}-1\right)-e_{i_{j}}\right)\left[A \cup \Gamma_{B^{\prime}}(A)\right] \\
=B_{A}-N\left(\mathcal{R}, i_{j}-1\right)-e_{i_{j}}=B_{A}-\left(N\left(\mathcal{R}, i_{j}-1\right) \cap E\left(B_{A}\right)\right)-e_{i_{j}} \\
=B_{A}-N\left(\mathcal{R}_{k}, j-1\right)-e_{i_{j}} .
\end{aligned}
$$

Por outro lado

$$
\begin{aligned}
B_{k}\left(\mathcal{R}_{k}, j-1\right)-e_{i_{j}}= & \left(B_{A}-r_{k}\right)\left(\mathcal{R}_{k}, j-1\right)-e_{i_{j}}=B_{A}\left(\mathcal{R}_{k}, j-1\right)-r_{k}-e_{i_{j}} \\
& =B_{A}-N\left(\mathcal{R}_{k}, j-1\right)-r_{k}-e_{i_{j}}=B_{A}^{\prime}-r_{k} .
\end{aligned}
$$

Do Lema 4.3 temos

$$
\begin{aligned}
& B_{A}^{\prime}-r_{k} \text { não tem }\left(f_{A}-l_{k}\right) \text {-fator esquerdo se e somente se } \\
& B_{A}^{\prime} \text { não tem } f_{A} \text {-fator esquerdo, }
\end{aligned}
$$

o que, por (4.8) e (4.13), é o mesmo que

$B_{k}\left(\mathcal{R}_{k}, j-1\right)-e_{i_{j}}$ não tem $f_{k}$-fator esquerdo se e somente se $B_{A}^{\prime}$ não tem $f_{A}$-fator esquerdo.

Em resumo: de (4.9), (4.11), (4.12) e (4.14)

$a_{j}^{\prime}=\operatorname{sim}$ se e somente se $B_{k}\left(\mathcal{R}_{k}, j-1\right)-e_{j}^{\prime}$ não tem $f_{k}$-fator esquerdo,

o que é o mesmo que dizer que $\mathcal{R}_{k}$ é uma realização de $\mathcal{F}\left(B_{k}, f_{k}\right)$ na qual a ocultadora segue a Estratégia $\mathcal{S}$.

Retornando ao argumento principal, temos $0<f_{k}\left(V_{1}\left(B_{k}\right)\right)=f(A)-1<f\left(V_{1}(B)\right)$, e assim, podemos aplicar a hipótese de indução à realização $\mathcal{R}_{k}$ de $\mathcal{F}\left(B_{k}, f_{k}\right)$, e concluir que, para algum $l \in V_{1}\left(B_{k}\right)$ temos

$$
\partial_{B_{k}}(l) \subseteq Q\left(\mathcal{R}_{k}\right)
$$


Como a definição de $B_{k}$ implica que $\partial_{B}(l) \subseteq \partial_{B_{k}}(l) \cup Q(\mathcal{R}, k)$, temos $\partial_{B}(l) \subseteq \partial_{B_{k}}(l) \cup Q(\mathcal{R}, k) \subseteq Q(\mathcal{R})$. 


\section{Capítulo 5}

\section{Comentários Finais}

O problema de otimização de consultas com predicados caros tem recebido alguma atenção por parte da comunidade de bases de dados (veja, por exemplo, Avnur and Hellerstein (2000), Bouganim, Fabret, Porto, and Valduriez (2001), Chaudhuri and Shim (1993), Hellerstein (1998), Laber, Parekh, and Ravi (2002), Porto (2001)). A maioria das propostas, entretanto não leva em conta o fato de que o valor de um atributo pode aparecer em diferentes tuplas, a fim de decidir como executar a consulta (veja, por exemplo, Bouganim et al. (2001), Chaudhuri and Shim (1993), Hellerstein (1998)). Noutras palavras, estas propostas somente estudam aquelas instâncias $(G, \delta, \gamma)$ do OD $k$ onde $G$ é um emparelhamento. Por exemplo, a principal referência neste assunto, o algoritmo de "Migração de Predicados" ("Predicate Migration") proposto por Hellerstein (1998), pode ser visto como um algoritmo otimamente competitivo para a variante do OD $k$ onde o hipergrafo $G$ dado como entrada é um emparelhamento, a probabilidade de um vértice em $V_{i}(G)$ ser verdadeiro é conhecida de antemão, e o objetivo é computar uma avaliação de custo esperado mínimo. A idéia de processar o hipergrafo induzido pela relação formada pela base de dados aparece pela primeira vez com Porto (2001). Nenhuma análise teórica é feita, entretanto.

Laber et al. (2002) e Bouganim et al. (2001) estudam o OD2d no modelo não-preemptivo. Laber et al. (2002) prova uma cota inferior de 2 para a competitividade de algoritmos determinísticos e de 1.5 para a competitividade de algoritmos aleatorizados. Também apresenta um algoritmo aleatorizado polinomial de competitividade $8 / 3$ e um algoritmo linear de competitividade 2 para o caso particular do OD2d no qual todos os vértices 
tem o mesmo custo.

Nesta parte do trabalho restringimos nossa atenção a consultas conjuntivas. Consultas muito mais gerais poderiam ser consideradas. Por exemplo, $\bar{\delta}: E(G) \rightarrow\{0,1\}$ poderia ser qualquer fórmula cálculo proposicional de primeira ordem envolvendo os predicados representados por $\delta$. Charikar, Fagin, Guruswami, Kleinberg, Raghavan, and Sahai (2002) consideram o problema da avaliação para consultas que podem ser representadas por uma "árvore AND/OR" sobre um conjunto de variáveis, onde o custo da avaliação de cada variável pode ser diferente. O contexto para consulta de informação cara proposto aí pode ser visto como uma versão restrita do problema descrito neste parágrafo, onde a entrada é um hipergrafo com uma única aresta.

Por fim observamos que para diferentes classes de hipergrafos $k$-partidos, o que inclui árvores e grafos com fluxos perfeitos, melhores cotas foram obtidas Feder, Motwani, O'Callaghan, Panigrahy, and Thomas (2003).

Dentre os problemas que permanecem abertos, destacamos os seguintes.

1. Existe um algoritmo aleatorizado para OD $k \mathrm{~s}$, ainda que exponencial, de competitividade $(k+1) / 2$ ? Não se sabe a resposta sequer para o caso $k=2$.

2. Existe um algoritmo polinomial para ODkd com deficiência $k$ para o modelo não-preemptivo? 


\section{Referências Bibliográficas}

N. Alon, B. Bollobás, G. Brightwell, and S. Janson. Linear extensions of a random partial order. Ann. Appl. Probab., 4:108-123, 1994. URL http://www.math.uu.se/ svante/papers/sj95.ps.

E. Arkin, H. Meijer, J. Mitchell, D. Rappaport, and S. Skiena. Decision trees for geometric objects. Internat. J. Comput. Geom. Appl., 8:343-363, 1998.

R. Avnur and J. M. Hellerstein. Eddies: continuously adaptive query processing. In Weidong Chen, Jeffery Naughton, and Philip A. Bernstein, editors, Proceedings of the 2000 ACM SIGMOD International Conference on Management of Data: May 16-18, 2000, Dallas, Texas, volume 29(2) of SIGMOD Record (ACM Special Interest Group on Management of Data), pages 261-272, New York, NY 10036, USA, 2000. ACM Press.

Y. Ben-Asher, E. Farchi, and I. Newman. Optimal search in trees. SIAM Journal on Computing, 28(6):2090-2102, December 1999. ISSN 0097-5397 (print), 1095-7111 (electronic).

B. Bollobás and G. Brightwell. The width of random graph orders. Math. Sci., 20(2):69-90, 1995. ISSN 0312-3685.

B. Bollobás. Extremal Graph Theory. Academic Press, London, 1 edition, 1978. ISBN 0-12-111750-2.

L. Bouganim, F. Fabret, F. Porto, and P. Valduriez. Processing queries with expensive functions and large objects in distributed mediator systems. In Proc. 17th Intl. Conf. on Data Engineering, April 2-6, 2001, Heidelberg, Germany, pages 91-98, 2001.

R. Carmo, J. Donadelli, Y. Kohayakawa, and E. Laber. Searching in random partially ordered sets. In LATIN: Latin American Symposium on Theoretical Informatics, 2002. 
R. Carmo, J. Donadelli, Y. Kohayakawa, and E. Laber. Searching in random partially ordered sets. Theoretical Computer Science, 321:41-57, 2004a.

R. Carmo, T. Feder, Y. Kohayakawa, E. Laber, R. Motwani, L. O'Callaghan, R. Panigrahy, and D. Thomas. A two-player game on graph factors. Technical Report RT-MAC 2004-05, IME-USP, 2004b.

M. Charikar, R. Fagin, V. Guruswami, J. Kleinberg, P. Raghavan, and A. Sahai. Query strategies for priced information. J. Comput. System Sci., 64(4):785-819, 2002. ISSN 0022-0000. Special issue on STOC 2000 (Portland, OR).

S. Chaudhuri and K. Shim. Query optimization in the presence of foreign functions. In Proc. 19th Intl. Conf. on Very Large Data Bases, August 24-27, 1993, Dublin, Ireland, pages 529-542, 1993.

W. J. Cook, W. H. Cunningham, W. R. Pulleyblank, and A. Schrijver. Combinatorial Optimization. John Wiley, New York, 1997.

T. Feder, R. Motwani, L. O'Callaghan, R. Panigrahy, and D. Thomas. Online distributed predicate evaluation. Technical Report 2003-81, Stanford University, 2003. URL http://dbpubs.stanford.edu/pub/2003-81.

J. M. Hellerstein. Optimization techniques for queries with expensive methods. ACM Transactions on Database Systems, 23(2):113-157, June 1998. ISSN 0362-5915.

L. G. Holanda. Algoritmos e novos limites para busca em conjuntos parcialmente ordenados. Master's thesis, COPPE-UFRJ, 2001. (In Portuguese).

L. Hyafil and R. L. Rivest. Constructing optimal binary decision trees is NPcomplete. Information Processing Letters, 5(1):15-17, May 1976. ISSN 0020-0190.

S. Janson, T. Luczak, and A. Rucinski. Random Graphs. Wiley-Interscience Series in Discrete Mathematics and Optimization. John Wiley \& Sons, Chichester-New York-Weinheim-Brisbane-Singapore-Toronto, 2000.

J. H. Kim and B. Pittel. On tail distribution of interpost distance. Journal of Combinatorial Theory Series B, 80:49-56, 2000.

D. J. Kleitman and B. L. Rothschild. Asymptotic enumeration of partial orders on a finite set. Trans. Amer. Math. Soc., 205:205-220, 1975. 
D. E. Knuth. The Art of Computer Programming, Vol 3, Sorting and Searching. Addison-Wesley, Reading, USA, 2 edition, 1998. ISBN 0-20189685-0.

E. Laber, O. Parekh, and R. Ravi. Randomized approximation algorithms for query optimization problems on two processors. In Proceedings of ESA 2002, pages 136-146, Rome, Italy, September 2002.

E. Laber, R. Carmo, and Y. Kohayakawa. Querying priced information in databases: The conjunctive case. Technical Report 05, IME-USP, São Paulo, Brasil, July 2003.

E. Laber, R. Carmo, and Y. Kohayakawa. Querying priced information in databases: The conjunctive case. Lecture Notes in Computer Science, 2976:6-15, 2004. ISSN 0302-9743.

N. Linial and M. Saks. Searching ordered structures. Journal of Algorithms, 6(1):86-103, March 1985a.

N. Linial and M. Saks. Every poset has a central element. Journal of Combinatorial Theory, Series A, 40:195-210, 1985b.

M. J. Lipman and J. Abrahams. Minimum average cost testing for partially ordered components. IEEE Transactions on Information Theory, 41(1): 287-291, Jan 1995.

C. H. Papadimitriou. Computational Complexity. Addison-Wesley, New York, 1994. ISBN 0-201-53082-1.

F. Porto. Estratégias para a Execução Paralela de Consultas em Bases de Dados Científicos Distribuídos (Strategies for the Parallel Execution of Queries in Distributed Scientific Databases). PhD thesis, Departamento de Informática, PUC-Rio, April 2001.

V. V. Vazirani. Approximation Algorithms. Springer-Verlag, 2001. 


\section{Índice de Definições}

árvore, 4

de busca de uma estratégia, 7

para uma ordem, 18

altura

de uma ordem, 14

anti-cadeia de uma ordem, 14

arco, 13

aresta

de um grafo, 13

de um hipergrafo, 53

falsa, 61

verdadeira, 61

avaliação, 60

Busca

Binária Parcial, 38

com Informação Parcial (BOIP), 47

cadeia de uma ordem, 14

camada

abaixo de uma camada em uma ordem, 37

acima de uma camada em uma ordem, 37

de uma ordem, 37

mediana de uma ordem, 37

caminho em uma árvore, 4

classe

direita de vértices de um grafo

bipartido, 93

esquerda de vértices de um grafo

bipartido, 93

cobertura

de um elemento por outro numa

ordem, 14

de um grafo, 54 mínima de um grafo, 54

comparáveis (elementos) em uma ordem, 14

competitividade

(razão de), 63

de um algoritmo, 64

de um algoritmo aleatório, 64

de uma avaliação, 63

contra-jogada de um jogo, 4

custo

de uma avaliação, 60

distribuído de um conjunto de vértices, 60

distribuído mínimo de uma instância do $\mathrm{OD} k, 60$

seqüencial de um conjunto de vértices, 60

seqüencial mínimo de uma instância do $\mathrm{OD} k, 60$

diagrama de Hasse, 15

dimensão de um hipergrafo, 53

duração de um jogo, 4

emparelhamento, 53

entre outros dois (elemento) numa ordem, 14

espaço de probabilidades uniforme, 4

estratégia

de busca para um jogo de esconder, 5

de evasão para um jogo de esconder, 5

para um jogo de esconder, 5

estrela de um vértice, 53

evento 
assintoticamente quase certo, 15

assintoticamente raro, 15

filho

de uma árvore, 4

direito de uma árvore, 4 esquerdo de uma árvore, 4

filtro de uma ordem, 14 gerado por um conjunto, 14 gerado por um elemento, 14

grafo, 13

bipartido, 53

bipartido completo, 53

dirigido, 13

hipergrafo 2-uniforme, 53

grau de um vértice

num grafo, 13

num hipergrafo, 53

hipergrafo, 53

$k$-partido, 53

$k$-uniforme, 53

uniforme, 53

ideal de uma ordem, 14

gerado por um conjunto, 14

gerado por um elemento, 14

incidência de uma aresta em um vértice

de um grafo, 13

de um hipergrafo, 53

incomparáveis (elementos) em uma ordem, 14

jogada de um jogo, 4

lance de um jogo, 4

largura de uma ordem, 14 maior que (relação entre elementos numa ordem), 13

maximal (elemento) de uma ordem, 14

menor que (relação entre elementos numa ordem), 13

modelo

probabilístico, 15

uniforme de ordens aleatórias, 33

ocultadora de um jogo de esconder, 4

ordem, 13

aleatória, 29

dual de uma ordem, 14

grafo-aleatória, 30

induzida por um conjunto, 14

parcial, 13

total, 14

tripartida, 33

pergunta em um jogo de esconder, 4

pertinência de um vértice a uma aresta, 53

ponta de uma aresta, 13

posto

$k$-ésimo de uma ordem, 14

conjunto dos ... de uma ordem, 14

de uma ordem, 14

procurador de um jogo de escon-

$$
\text { der, } 4
$$

rara, 15

típica, 15

ramo

de uma árvore, 4

direito de uma árvore, 4 
realização de um jogo, 4

resposta em um jogo, 4

segmento

0-ésimo de uma ordem, 14

último de uma ordem $P, 15$

de uma ordem, 14

seleção (operador do modelo relacional de bases de dados), 57

subgrafo

gerado por um conjunto de arestas, 54

induzido por um conjunto de vértices, 13

suporte de um espaço de probabilidades, 4

vértice

de um grafo, 13

de um grafo dirigido, 13

de um hipergrafo, 53

falso, 61

verdadeiro, 61

vértice isolado

de um hipergrafo, 53

vizinhança, 53

de um conjunto de vértices em um grafo, 13

de um conjunto de vértices em um hipergrafo, 53

de um vértice em um grafo, 13

de um vértice em um hipergrafo, 53 